UNIVERSITÉ DU QUÉBEC À CHICOUTIMI

ESSAI DOCTORAL PRÉSENTÉ À

L'UNIVERSITÉ DU QUÉBEC À CHICOUTIMI

COMME EXIGENCE PARTIELLE

DU DOCTORAT EN PSYCHOLOGIE (D.Ps.)

\author{
PAR \\ AUDREY POTVIN
}

ÉTUDE EXPLORATOIRE DESCRIPTIVE DE L'EFFICACITÉ DE DIFFÉRENTES MÉTHODES DE GUIDAGE INFORMATISÉ LORS DE LA RÉALISATION D'ACTIVITÉS DE PRÉPARATION D'UN CAFÉ ET D'UNE RÔTIE ET D'EMBALLAGE D'UN CADEAU AUPRÈS DE TROIS PATIENTS ALZHEIMER EN STADE MODÉRÉ 
Table des matières ........................................................................................

Liste des tableaux ........................................................................................

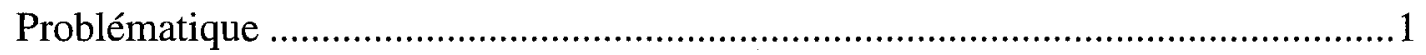

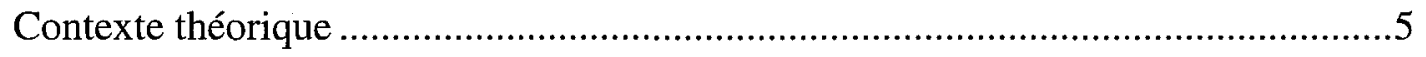

La maladie d'Alzheimer......................................................................6

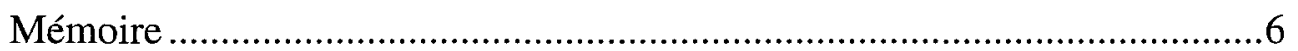

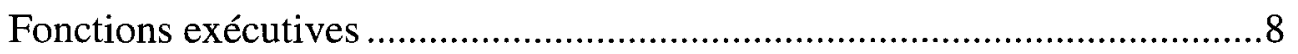

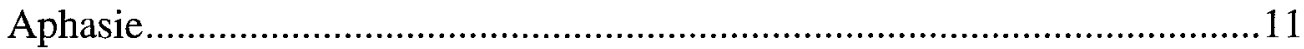

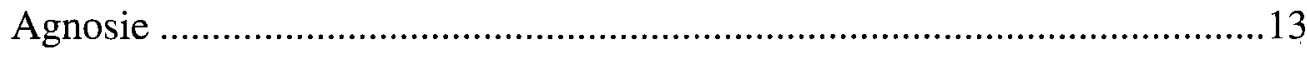

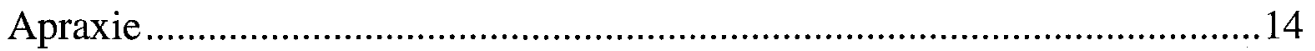

La nutrition comme activité de la vie quotidienne : impacts de la MA ..............16

Assistance technologique intelligente ...................................................... 18

Le modèle PPH et l'évolution hétérogène de la MA : impacts sur les

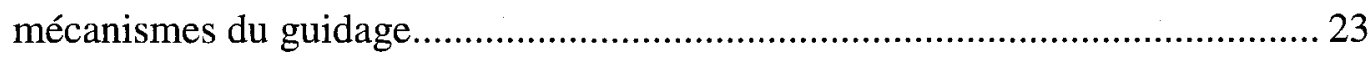

But de l'étude et objectifs de recherche .....................................................24

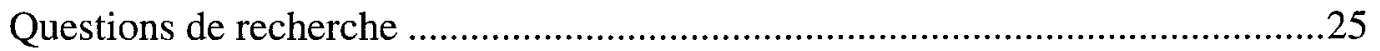

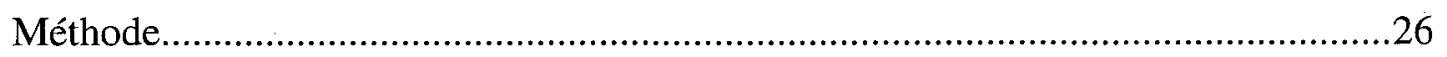

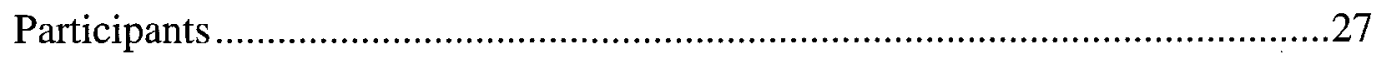

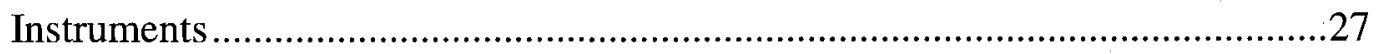

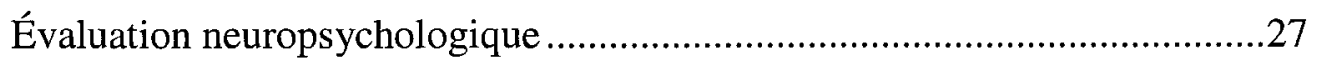

Global Deterioration Scale ............................................................28 


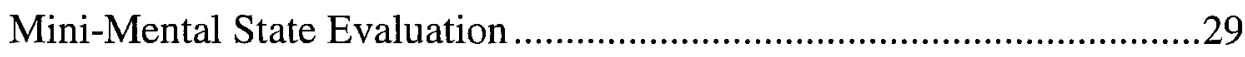

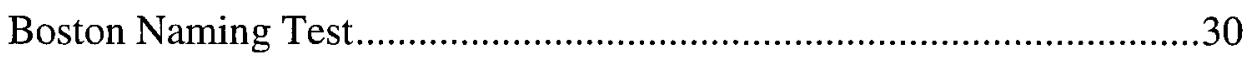

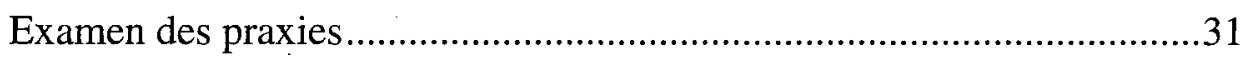

Protocole d'évaluation des gnosies visuelles ............................................31

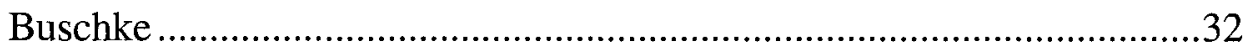

Trail Making Test...............................................................................

Test du dessin de l'horloge .......................................................................34

Naturalistic Action Test ............................................................................

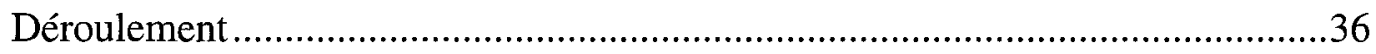

Établissement de scripts d'activité de tâches tirées du NAT ..........................36

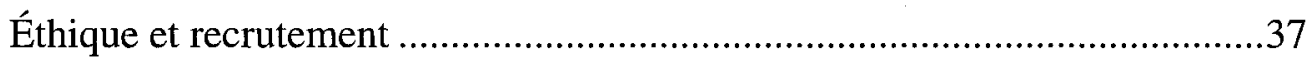

$1^{\text {re }}$ étape : signature du formulaire de consentement.....................................37

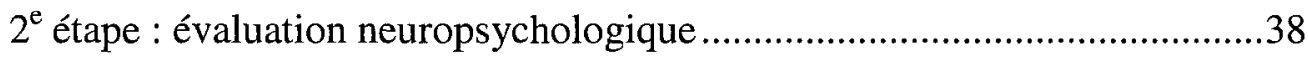

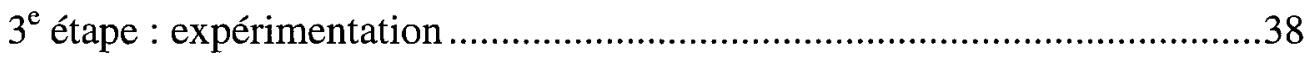

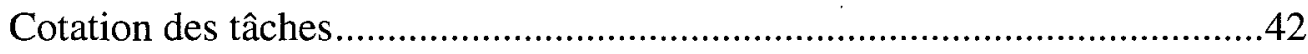

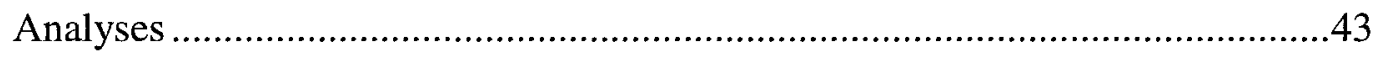

Définition des variables de comparaison de groupes ........................................44

Définition des variables des analyses descriptives.........................................45

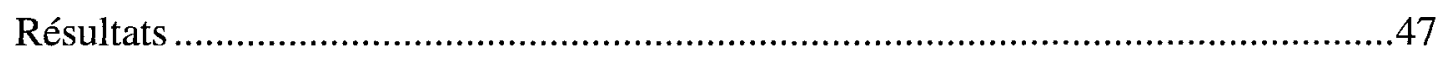

Description de l'échantillon ..........................................................................48

Description des participants et évaluation neuropsychologique ...........................50

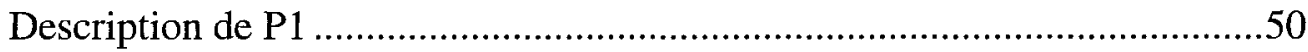




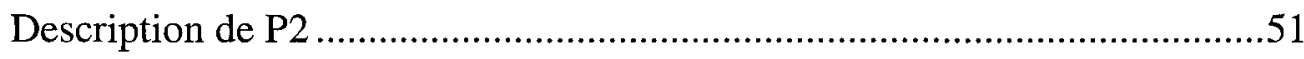

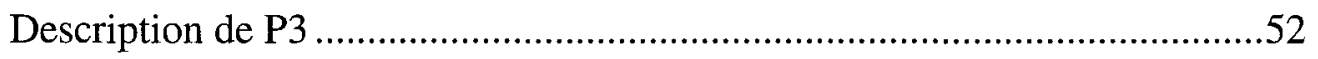

Expérimentation : tâche café et rôtie...................................................................53

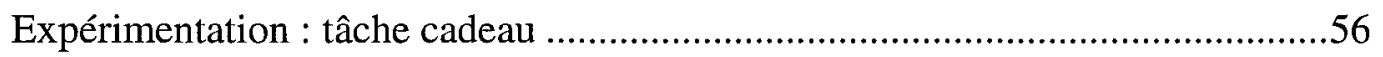

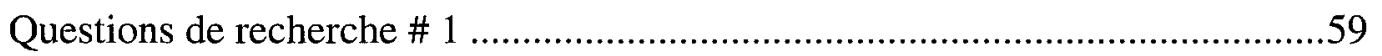

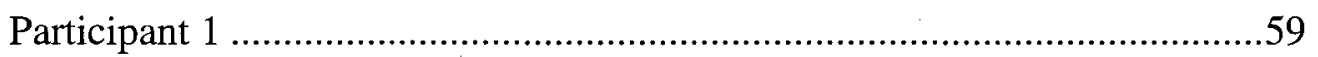

Participant 2 .

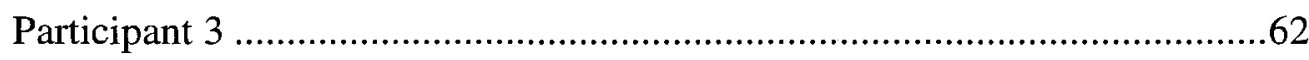

Résumé de l'efficacité des guidages .................................................................63

Question de recherche \#2 ...........................................................................64

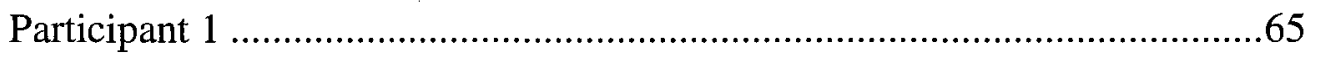

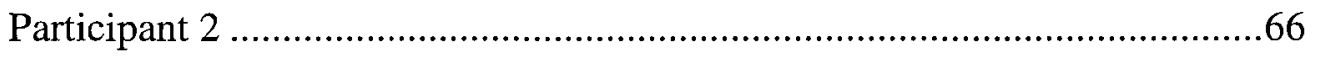

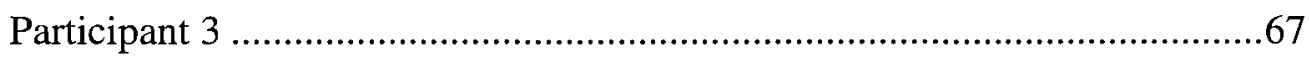

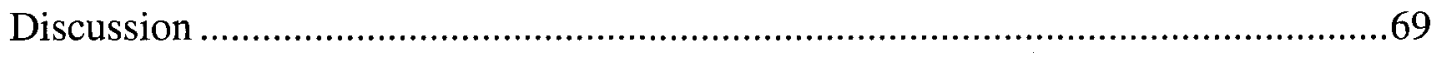

Comparaison des performances : groupe contrôle et groupe clinique ...................70

Expérimentation : tâche café et rôtie.............................................................70

Expérimentation : tâche cadeau ....................................................................73

Effet d'apprentissage

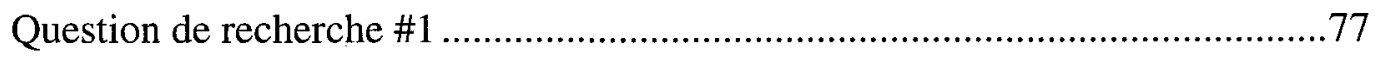

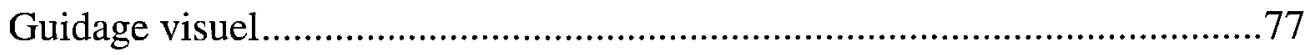

Guidage verbal et audiovisuel.....................................................................78

Question de recherche \# 2 .......................................................................... 
Forces et faiblesses de l'étude.

Analyses des conséquences de la recherche et retombées possibles.

Conclusion

Références .95

Appendice A : Formulaire de consentement ..........................................................106

Appendice B : Questionnaire sociodémographique ...................................................113

Appendice $\mathrm{C}$ : Description des interventions auprès du participant ...........................115

Appendice D : Exemple d'ordre de présentation des méthodes de guidage ...............117

Appendice E : Étapes d'introduction des guidages dans la séquence : tâche café

et rôtie.

Appendice F : Étapes d'introduction des guidages dans la séquence : tâche cadeau. Appendice G : Modèle du traitement de l'information selon Wickens (1992) 


\section{Liste des tableaux}

\section{Tableau}

1 Temps écoulé entre l'évaluation neuropsychologique et l'expérimentation........48

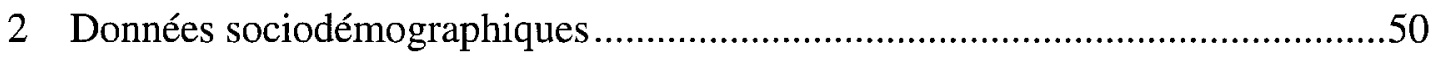

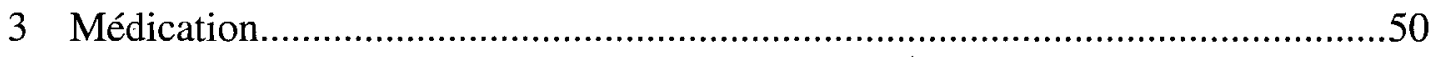

4 Résultats de l'évaluation neuropsychologique........................................53

5 Nombre d'étapes tâche café et rôtie .......................................................55

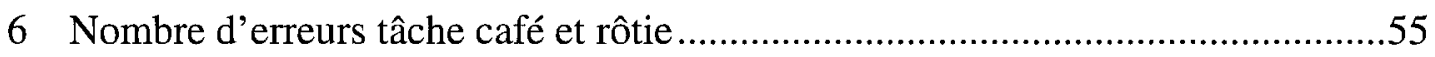

7 Temps de réalisation tâche café et rôtie ....................................................55

8 Comparaison du groupe contrôle et du groupe clinique tâche café et rôtie ..........56

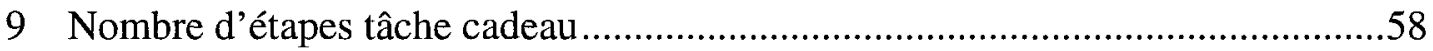

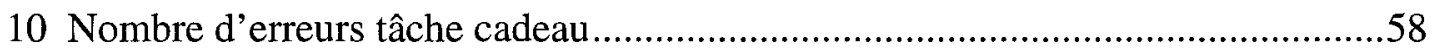

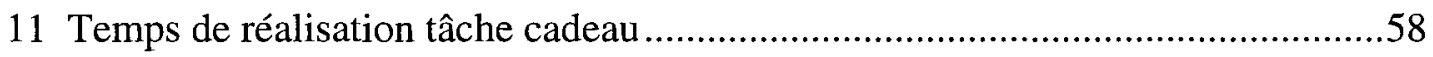

12 Comparaison du groupe contrôle et du groupe clinique tâche cadeau.................59

13 Efficacité des guidages selon les participants ..........................................64 
Problématique 
La maladie d'Alzheimer (MA) est la cause la plus fréquente de démence dans les pays occidentaux industrialisés et les coûts reliés à cette maladie approchent les 5,5 milliards de dollars par année au Canada (DeKosty \& Orgozogo, 2001; Diamond, 2006). De plus, la Société Alzheimer du Canada (2009) s'attend à ce que le nombre de personnes atteintes double en l'espace d'une génération. La MA se caractérise par des modifications neurophysiologiques de nature pathologique. Ces modifications provoquent un déclin cognitif d'évolution constante (Giffard, Desgranges, \& Eustache, 2008). Ce déclin peut prendre diverses formes et affecter certaines sphères cognitives telles que les fonctions exécutives et instrumentales. Les atteintes cognitives peuvent être importantes et elles requièrent une prise en charge croissante au fil de l'évolution de la maladie, tant par le système de santé que par l'entourage des personnes atteintes (Derouesné, 2006). Les répercussions engendrées par le déclin cognitif peuvent être sévères en ce qui concerne l'autonomie de l'individu, plus précisément sur la réalisation d'activités de la vie quotidienne (AVQ) et de la vie domestique (AVD). La perturbation des AVQ et des AVD augmente le risque d'accident à domicile, de même que le fardeau des aidants, et est en lien avec l'institutionnalisation des patients atteints de MA (Mahurin, DeBettignies, \& Pirozzolo, 1991). Afin de pallier à la pression issue d'une demande croissante de soins qui incombe souvent aux proches (aidants) de la personne atteinte, des travaux de recherche s'effectuent actuellement afin de développer des mécanismes de compensation des déficits cognitifs prenant la forme d'agents intelligents 
(de type prothétique) qui seraient en mesure de soutenir les patients atteints de MA dans la réalisation de leurs AVQ et AVD. Ces agents prothétiques et intelligents visent notamment à compenser temporairement les altérations cognitives engendrées par la MA, ce qui permet une prolongation du maintien à domicile. La charge et la demande mise sur les aidants pour les soins pourraient ainsi potentiellement être réduites, ce qui autoriserait un allongement de la période de vie à domicile (Bouchard, Bouzouane, \& Giroux, 2007). Afin de parvenir à cet objectif, différentes techniques peuvent être utilisées, dont l'émission de messages-guides ayant comme visée d'accompagner l'individu dans la réalisation d'AVQ (alimentation, soins personnels, habillement, etc.). Mihailidis, Barbenel et Fernie (2004) ont démontré, dans une étude d'efficacité auprès de 10 patients atteints de démence modérée à sévère, que le nombre d'étapes pour la complétion d'une tâche spécifique que les participants sont en mesure de réaliser sans l'aidant est accru de $25 \%$ lors de l'utilisation d'une méthode de guidage verbale informatisée. Cependant, une autre étude d'efficacité réalisée auprès d'un participant atteint de MA sévère montre que, malgré le fait que l'utilisation d'un guidage verbal informatisé augmente le niveau d'autonomie, seulement un faible pourcentage des guidages s'est avéré efficace (Mihailidis, Boger, Canido, \& Hoey, 2007). Ces résultats suggèrent que différents facteurs semblent agir sur l'efficacité du guidage informatisé, dont le médium par lequel le guidage est transmis. À ce jour, différentes équipes de recherche ont utilisé plusieurs types de guidages informatisés auprès d'échantillons de personnes atteintes de démence, mais peu d'entre elles se sont attardées à en étudier systématiquement les effets différentiels. Ces guidages informatisés peuvent prendre la 
forme de commandes vocales comprenant des niveaux variables de précision (Mihailidis et al., 2007) ou encore de stimulations visuelles (images, vidéos, lumières) ou sonores (Pigot, Mayers, \& Giroux, 2003). Il est donc primordial d'effectuer des études concernant l'efficacité de diverses formes de guidages informatisés afin d'optimiser leurs effets et impacts auprès des patients. C'est pourquoi ce projet de recherche, réalisé sous forme d'observation de base du comportement prenant la forme de trois descriptions cliniques, se penche sur cette problématique de premier plan et a pour but de décrire l'efficacité de trois types de guidages informatisés auprès de participants atteints de MA en stade modéré. Les méthodes de guidage utilisées sont présentées sous forme visuelle (iconique), auditive avec contenu verbal, ainsi qu'une combinaison des deux. De plus, le volet neuropsychologique (atteintes des fonctions instrumentales et exécutives) sera exploré en tant que facteur pouvant moduler les réponses des patients et donc, l'efficacité du guidage informatisé. 
Contexte théorique 


\section{La maladie d'Alzheimer}

La maladie d'Alzheimer consiste en «un déclin progressif et irrémédiable du fonctionnement cognitif, caractérisé par la dégénérescence du tissu cérébral, avec une perte des neurones, le développement de plaques séniles et la présence de dégénérescences neurofibrillaires » (Encyclopédie médicale - Le Manuel Merck, 2007). Elle se traduit par une altération progressive du fonctionnement cognitif qui engendre des troubles fonctionnels, sociaux et physiques (Dartigues, Berr, Helmer, \& Letenneur, 2002). Selon les critères du DSM-IV-TR (American Psychological Association, 2004), le patient doit présenter une altération de la mémoire ainsi qu'une ou plusieurs des perturbations cognitives suivantes : dysfonctions exécutives, aphasie, agnosie ou apraxie. Les fonctions instrumentales (mémoire, langage, praxies, gnosies) sont ainsi touchées dans la MA, de même que les fonctions exécutives (Giffard et al., 2008).

\section{Mémoire}

Les troubles de la mémoire sont parmi les symptômes les plus précoces de la MA (Nestor, Scheltens, \& Hodges, 2004). En effet, plusieurs systèmes mnésiques sont touchés et il existe une grande variabilité de présentations cliniques, tant au niveau de la nature des processus déficitaires que de la progression des déficits (Eustache \& Desgranges, 1995; Perry \& Hodges, 2000). La littérature s'accorde pour affirmer que les déficits en mémoire épisodique, qui touchent autant la mémoire verbale que non verbale, 
représentent un des signes précurseurs de la MA (Guégen, Chauvel, \& Touchon, 2005). Il serait d'ailleurs possible de détecter de tels déficits à un stade pré-clinique de la maladie. Une perturbation du processus d'encodage, de même qu'une difficulté à consolider de nouvelles informations seraient à l'origine des difficultés en mémoire épisodique vécues par les personnes atteintes. Ces déficits entraîneraient l'apparition progressive d'une amnésie antérograde (incapacité à former de nouveaux souvenirs) au cours des stades léger à modéré de la maladie (Salmon \& Hodges, 2001). Cette forme d'amnésie progresse insidieusement (Zec \& Burkett, 2005), puis peut atteindre une phase plateau pendant plusieurs années avant de subir un déclin significatif ayant des conséquences importantes sur le fonctionnement de l'individu.

Les manifestations des troubles de mémoire peuvent prendre la forme d'une désorientation temporelle, spatiale et personnelle, d'oublis d'évènements récents et d'informations récentes ou encore de difficultés dans l'apprentissage de nouvelles informations (Giffard et al., 2008). Zec et Burkett (2005) indiquent qu'au stade modéré de la MA, l'ensemble des composantes d'un apprentissage de type déclaratif (soit l'encodage, la consolidation, le rappel immédiat et différé ainsi que la reconnaissance) est atteint de façon importante. Par la suite, dans les stades modéré à avancé de la maladie, s'installe progressivement une amnésie rétrograde. Dans la MA, cette amnésie suit un gradient temporel de détérioration (gradient temporel de Ribot) (Ribot, 1882) signifiant que les informations anciennes sont conservées en mémoire plus longtemps. L'amnésie rétrograde progressera jusqu'à ce que les déficits soient considérés sévères. 
La mémoire implicite et la mémoire sémantique sont touchées dans la MA. Ces deux systèmes demeurent préservés plus longtemps que la mémoire épisodique, malgré que des déficits subtils puissent être détectés dès le stade modéré de la maladie (Hodges et Patterson, 1997). Pour la mémoire sémantique, ces difficultés peuvent prendre la forme d'un discours spontané vague, de mots imprécis et de circonlocutions ou encore d'une difficulté à identifier des personnes (Giffard et al., 2008).

La mémoire immédiate est atteinte dès le stade initial de la maladie et les symptômes progressent avec l'évolution des stades. Il en est de même pour la mémoire de travail, qui concerne la capacité à manipuler mentalement des informations (Becker, 1988). Les patients atteints peuvent également éprouver de la difficulté à effectuer deux tâches simultanément, élément faisant référence à la mémoire de travail et l'attention partagée. Les déficits en mémoire immédiate et en mémoire de travail pourraient restreindre le nombre d'informations transférées en mémoire à long terme. Finalement, la mémoire procédurale demeure relativement intacte dans la MA (Welsh-Bohmer \& Warren, 2006).

\section{Fonctions exécutives}

Les fonctions exécutives représentent des « fonctions de haut niveau (des fonctions de contrôle) impliquées dans de nombreuses formes d'activités cognitives » (Allain \& Le Gall, 2008, p.9). Elles sont responsables notamment de la planification, de l'organisation et de la synchronisation des actions complexes. D'autres processus cognitifs supérieurs tels que le jugement, l'abstraction, la résolution de problèmes ainsi 
que le raisonnement sont également impliqués dans le fonctionnement exécutif (Bherer, Belleville, \& Hudon, 2004).

Les fonctions exécutives accusent généralement un déclin chez les sujets normaux vieillissants. Dans la MA, les fonctions exécutives sont également touchées, mais de façon plus importante et plus fréquemment que dans le vieillissement normal (Bherer et al., 2004). Malgré que les manifestations d'une problématique exécutive puissent être plus subtiles que la problématique mnésique, l'atteinte de ces fonctions est précoce (Swanberg, Tractenberg, Mohs, Thal, \& Cummings, 2004) et importante (Perry \& Hodges, 2000). De plus, l'atteinte des fonctions exécutives ne serait pas uniforme d'un patient à l'autre. Par exemple, un patient pourrait présenter une atteinte relativement hâtive de ces fonctions, mais qui évoluera lentement. Chez un autre patient, les fonctions exécutives peuvent demeurer apparemment intactes pendant plusieurs années, puis se détériorer rapidement. Les recherches se poursuivent donc afin de préciser la nature des déficits en jeu ainsi que les facteurs communs (Giffard et al., 2008).

L'évaluation neuropsychologique de personnes atteintes de MA témoigne que plusieurs composantes du fonctionnement exécutif peuvent être touchées. Levinoff, Murtha, Li, et Chertkow (2004) énoncent qu'une sensibilité à l'interférence serait présente au stade précoce de la maladie et augmenterait avec la progression des stades (déficit d'inhibition). La perturbation exécutive pourrait également entraîner des difficultés de flexibilité mentale et des persévérations comportementales (Paolo, Axelrod, 
Troster, Blackwell, \& Koller, 1996) ainsi que des difficultés de raisonnement, d'abstraction, de conceptualisation et de résolution de problèmes (Swanberg et al., 2004). Les facultés attentionnelles s'avèreraient, quant à elles, déficitaires au niveau du contrôle et de la direction de l'attention (Bherer et al., 2004). Selon Logie, Della Sala, Cocchini, \& Baddeley (2004), la mémoire de travail serait touchée en tâche double (faisant appel aux capacités d'attention divisée), de même que lors de tâches de raisonnement mathématique (Cummings, 1990). Les personnes atteintes de MA peuvent donc éprouver une difficulté à réaliser des tâches complexes requérant le traitement simultané de plusieurs sources d'informations.

Les dysfonctions exécutives sont en lien avec l'augmentation du besoin de prise en charge chez les patients atteints (Royall et al., 1993). Il en découle que les manifestations cognitives pathologiques dues aux atteintes exécutives se répercutent rapidement dans la vie quotidienne des patients (Giovannetti, Libon, Buxbaum \& Schwartz, 2002) et ont des impacts négatifs non négligeables sur l'indépendance, l'autonomie et la sécurité de ces derniers, ainsi que sur la qualité de vie des aidants (Giffard et al., 2008; Potkin, 2002; Tekin, Fairbanks, O'Connor, Rosenberg, \& Cummings, 2001). Chen, Sultzer, Hinkin, Mahler et Cummings (1998) affirment également que les déficits exécutifs dans la MA engendrent une atteinte fonctionnelle ainsi qu'une incapacité à exécuter les AVQ. Les capacités de flexibilité cognitive, de résistance à la distraction, de résolution de problème et de jugement seraient également des facteurs clés dans la réalisation d'activités de tous les jours. Ainsi, il existe un risque 
réaliste que les personnes atteintes de MA puissent poser des gestes non appropriés aux circonstances ou être incapables d'effectuer des liens entre certains concepts acquis. Les déficits exécutifs seraient donc une bonne indication du niveau d'aide requis par le patient.

\section{Aphasie}

L'aphasie est une "altération acquise du langage, conséquence d'une lésion organique » (Verstichel \& Cambier, 2006, p. 476). Elle peut toucher autant les sphères d'expression que de compréhension, à l'écrit comme à l'oral. Dans la MA, les troubles du langage sont constants (Giffard et al., 2008) et sont les plus fréquents après les troubles de la mémoire (Derouesné, 2006). Ces derniers peuvent également être le symptôme prédominant pendant plusieurs années, tout comme ils peuvent être absents du tableau clinique au début de la maladie. Malgré l'hétérogénéité des manifestations des troubles du langage dans la MA, certaines constantes existent et permettent de caractériser les troubles les plus fréquemment observés (Giffard et al., 2008).

Selon Giffard et al. (2008), l'aphasie dans la MA est généralement décrite en trois stades évolutifs de gravité croissante. Le premier stade se rapproche de l'aphasie anomique ou amnésique. En effet, le patient peut être atteint d'un manque du mot accompagné de circonlocutions (moyen indirect, façon détournée d'exprimer une pensée) et de rares paraphasies sémantiques. La fluence verbale sémantique est appauvrie. Cependant, les troubles phonétiques, phonémiques ou syntaxiques sont absents et la 
compréhension est souvent préservée (Cardebat, Aithamon, \& Puel, 1995). À un stade plus avancé, le tableau clinique se rapproche de l'aphasie transcorticale sensorielle (Giffard et al., 2008) qui se présente comme une aphasie de Wernicke avec répétition; le langage est fluent, mais incompréhensible (Lechevalier \& Viader, 2008). Cela signifie que les symptômes décrits précédemment s'aggravent, le discours devient incohérent et de nombreuses paraphasies sémantiques et formelles sont observables. Des troubles de la compréhension apparaissent également. Le dernier stade est caractérisé par l'atteinte de l'ensemble des sphères linguistiques et se rapproche d'un tableau clinique d'aphasie globale (altération de l'ensemble des fonctions langagières). L'expression devient difficilement compréhensible avec des paraphasies phonémiques (transformation de la forme phonologique du mot), des persévérations ainsi que des néologismes (fabrication d'un mot nouveau ou utilisation d'un mot habituel avec une signification nouvelle). L'écholalie peut également être présente et attribuable aux capacités résiduelles de répétition.

Les atteintes au niveau du langage dans la MA mènent à des difficultés de la communication entre l'aidant, le patient et l'entourage; difficultés amplifiées avec l'évolution de la MA (Wilson et al., 2007). En ce qui a trait aux troubles de compréhension, il est important de présenter les informations une à la fois et de façon claire afin de maximiser le taux de réponse de la part du patient (Khosravi, 2003). Il est également admis que les troubles du langage dans la MA contribuent à la dépendance dans le fonctionnement quotidien (Tekin et al., 2001). 


\section{Agnosie}

L'agnosie représente « (...) un déficit de la reconnaissance en l'absence de troubles sensoriels élémentaires et linguistiques »(Giffard et al., 2008, p.786). Cette affection peut être de nature visuelle, auditive ou somesthésique. Dans la MA, l'agnosie visuelle serait la plus fréquente et toucherait $30 \%$ des cas avec, comme manifestation, la nonreconnaissance d'objets ou de visages familiers (aussi appelée prosopagnosie) (Derouesné, 2006). Il est admis que toutes les formes d'agnosies peuvent être retrouvées dans la MA. L'agnosie visuelle gêne le fonctionnement quotidien du sujet (Giffard et al., 2008) qui peut, par exemple, expérimenter de la difficulté à reconnaître une cuillère parmi un ensemble d'ustensiles de cuisine. La prosopagnosie augmente en fréquence avec l'évolution de la maladie et, au cours des derniers stades, le patient ne reconnaît plus ses proches. Quant à elle, l'agnosie auditive se manifeste par la non-reconnaissance de sons verbaux et non verbaux (voix, musique, son de l'environnement) pouvant être expliquée par un trouble d'origine centrale. L'anosognosie est la dernière forme d'agnosie pouvant se retrouver dans la MA. Elle se définit comme l'habileté réduite à reconnaître la présence ou à apprécier la sévérité des déficits au niveau sensoriel, moteur, cognitif et affectif. Ce trouble est fréquemment observé dans les stades avancés de la maladie (Antoine, Antoine, Guermonprez, \& Frigard, 2004), malgré le fait que le lien entre la sévérité de la démence et l'anosognosie ne soit pas encore clairement établi (Wagner, Spangenberg, Bachman, \& O’Connell, 1997). 
L'agnosie n'est pas sans impact sur la vie quotidienne des personnes atteintes de MA. En effet, il existe une association entre le statut fonctionnel et les performances visuospatiales au sein des échantillons de patients atteints de MA (Mahurin et al., 1991; Willis, Allen-Burge, Dolan, Bertrand, Yesavage, \& Taylor, 1998). De plus, la reconnaissance d'objets est un prédicteur significatif de la dépendance dans les AVQ (Jefferson, Barakat, Giovannetti, Paul \& Glosser, 2006; Tekin et al., 2001).

\section{Apraxie}

L'apraxie se définit comme étant une « incapacité à exécuter des actes qui exigent la mémorisation de schémas ou de séquences de mouvements » (Encyclopédie médicale Le manuel Merck, 2007). Dans la MA, ces troubles apparaissent suite à l'installation des troubles de la mémoire, du langage ainsi que des troubles spatiaux. Il a été démontré que les manifestations de l'apraxie sont indépendantes de la sévérité de l'atteinte cognitive (Lagha Pierucci, 1996), malgré que l'on reconnaisse que le trouble soit constant lorsque la démence est en phase sévère (Della Sala, Lucchelli, \& Spinnler, 1987). L'apraxie dans la MA se caractérise par des manifestations hétérogènes (Giffard et al., 2008) d'intensité variable d'un patient à l'autre, le sujet peut donc être atteint de différentes formes d'apraxies qui apparaissent à différents moments de la maladie (Derouesné, 2006). Les atteintes peuvent intéresser le système conceptuel (les plus fréquentes) et/ou le système de production du geste, selon les patients (Derouesné, 2006). Le système conceptuel est celui qui génère les représentations internes et abstraites de l'action, tandis que le système de production opère dans la réalisation concrète du geste. De façon parallèle, il 
existe plusieurs types d'apraxies soit : l'apraxie constructive qui est une perturbation des activités graphiques (dessin ou écriture) ou de la construction de motifs en deux ou trois dimensions à l'aide de cubes, de bâtonnets ou de pièces à assembler; l'apraxie idéomotrice (ou gestuelle) qui est une difficulté à effectuer des gestes concrets (comprend les gestes transitifs, qui miment l'usage d'un objet mais sans usage d'objet, et intransitifs, qui n'impliquent pas la manipulation d'objets); l'apraxie idéatoire qui concerne les troubles de l'utilisation des objets et les difficultés de séquençage de l'action et; l'apraxie mélokinétique (ou mélocinétique) qui est une désorganisation unilatérale du mouvement qui se manifeste par une négligence motrice ainsi que par la prévalence de positionnements toniques du membre (Botez-Marquard \& Boller, 2005). La littérature concernant la MA évoque principalement l'apraxie constructive qui se veut fréquente et souvent d'apparition précoce. Lors de la présence de ce trouble, le patient atteint peut avoir de la difficulté à dessiner ou encore à compléter un casse-tête. Finalement, malgré le fait que les troubles de réalisation gestuelle représentent un handicap majeur dans la MA (Lagha Pierucci, 1996), les travaux sur le sujet demeurent relativement peu nombreux (Viader, Eustache \& Lechevalier, 2000).

Les liens entre les manifestations apraxiques et les atteintes dans l'exécution des AVQ ont été peu explorés à ce jour (Bayard, Derouesné, \& Gély-Nargeot, 2006). Il est cependant reconnu que l'apraxie est un facteur important dans la perte d'autonomie liée aux AVQ puisque les patients atteints ne sont plus en mesure de retrouver les séquences d'action pourtant automatisées (Lechowski et al., 2003; Schwartz \& Buxbaum, 1997; 
Tekin et al., 2001). Par exemple, dans l'apraxie de l'habillage, le patient éprouve de la difficulté à mettre ses vêtements dans l'ordre adéquat, à les boutonner et à utiliser les fermetures éclair (Bayard et al., 2006). D’ailleurs, la plupart des AVQ nécessiteraient des habiletés intactes à exécuter des gestes simples (Lechowski et al., 2003). De plus, les atteintes du système conceptuel font en sorte que le patient éprouve de la difficulté à associer certains objets entre eux (par exemple, un marteau et un clou), ce qui mène à une sélection inappropriée des objets destinés à une tâche spécifique. Cette atteinte reflète la perte des connaissances reliée à l'utilisation de différents outils chez les patients MA (Raymer \& Ochipa, 1997).

La nutrition comme activité de la vie quotidienne : impacts de la MA

Les AVQ consistent en un ensemble d'activités de base qui sont réalisées chaque jour. Leur exécution requiert la mise en action des fonctions exécutives (jugement, flexibilité, planification/organisation), de l'attention, du langage, de la mémoire, des praxies, des fonctions visuoperceptives ainsi que de l'orientation temporelle et spatiale (Paquette, 2009). L'évolution de la MA conduit invariablement et précocement à une détérioration des habiletés à compléter les AVQ (Derouesné, 2006; Potkin, 2002). Les activités les plus élaborées, appelées «AVQ instrumentales », sont touchées en premier lieu; le patient éprouve alors de la difficulté à participer à des activités sociales ou de loisirs, à gérer ses finances, à utiliser des transports en commun, à préparer des repas, etc. Par la suite, les AVQ de base sont atteintes (s'habiller, se laver, manger, se déplacer, etc.). 
La nutrition fait partie à la fois de la catégorie des AVQ dite instrumentale et de la catégorie dite de base. En effet, selon la Classification québécoise de processus de production du handicap (Fougeyrollas, Cloutier, Bergeron, Côté, \& St-Michel, 1998), la planification des repas, l'achat de la nourriture ainsi que la préparation des aliments entrent tous dans le domaine de la nutrition et sont des activités complexes et élaborées. La prise des repas, quant à elle, chevauche les deux types d'activités (instrumentale et de base), car elle inclut autant la prise de repas à la maison qu'à l'extérieur (ex. au restaurant) ainsi que le fait d'utiliser des instruments pour se nourrir (assiettes, verres, ustensiles, etc.) ou de manger comme tel. Les activités de nutrition sont également en lien avec les habitudes reliées aux soins de santé en ce sens qu'une saine alimentation favorise le maintien d'une bonne santé tant physique que psychologique (Organisation mondiale de la santé, 2004). Les fonctions cognitives impliquées dans des AVQ de nutrition sont l'attention, les fonctions visuoperceptives, les fonctions exécutives (flexibilité, jugement, planification/organisation), le langage (compréhension écrite), la mémoire, les praxies et la motricité (Paquette, 2009).

L'AVQ nutrition est grandement touchée dans la MA et ce, en raison du fait que les différentes perturbations cognitives découlant de la maladie rendent difficile l'exécution des multiples composantes liées à cette AVQ. En premier lieu, les déficits sur les plans mnésique et exécutif entravent le volet instrumental de l'AVQ, c'est-à-dire la planification et la préparation des repas. Par la suite, avec l'évolution de la maladie, les patients peuvent souffrir d'une dénutrition liée à la réduction de la prise alimentaire 
(Giffard et al., 2008). Il est donc important d'élaborer des solutions afin de favoriser le maintien de l'autonomie de l'AVQ nutrition, mais également des autres AVQ et AVD, et ce aussi longtemps que possible. De cette façon, la pression de soins mise sur les proches qui assurent le maintien à domicile des patients sera potentiellement réduite.

\section{Assistance technologique intelligente}

L'important accroissement démographique des personnes âgées conduit à une augmentation des cas de démence, puisque l'âge est un facteur de risque en ce qui concerne la maladie d'Alzheimer (Dartigues et al., 2002). Il est reconnu que les personnes âgées atteintes de démence bénéficient du fait de demeurer dans des environnements familiers le plus longtemps possible (par exemple à leur domicile) (Cutchin, 2003). Dans ce contexte, le maintien à domicile devient un enjeu économique et sociétal d'une très grande importance (L'association québécoise d'établissements de santé et de services sociaux, 2007). Afin de mettre en place les conditions favorisant le maintien à domicile, la recherche se tourne présentement vers les technologies d'assistance qui offrent à cet égard un grand potentiel (Bouchard et al., 2007). L'approche du maintien à domicile, à l'aide de certaines techniques de guidage informatisé ${ }^{1}$, figure parmi l'ensemble des possibilités que propose l'assistance technologique. Ces techniques de guidage peuvent être réalisées au moyen d'une assistance informatisée en mesure de soutenir la réalisation d'AVQ en émettant des interventions lorsque le patient atteint de MA commet une erreur ou hésite dans la

\footnotetext{
${ }^{1}$ Afin d'alléger le texte, le terme guidage sera employé afin de désigner guidage informatisé
} 
réalisation d'une tâche. Des études ont démontré jusqu'à maintenant que l'utilisation de message-guides, fourni par un aidant, représente une stratégie efficace afin d'assister les individus atteints de démence avec des activités d'habillement, d'alimentation et de communication (Engelman, Altus, Mosier, \& Mathews, 2003; Perry, Galloway, Bottorf, \& Nixon, 2005). En s'inspirant de ces données, certains auteurs ont effectué des recherches sur l'efficacité de ces message-guides, mais cette fois-ci émis via un médium informatique. Le développement de telles technologies ne vise pas le remplacement des aidants, mais plutôt un soutien aux AVQ des patients qui permettra un allègement des tâches incombant aux aidants (Boger et al., 2006).

Mihailidis et al. (2004) ont démontré avec 10 participants que le guidage verbal informatisé, lors d'une tâche de lavage de mains, est efficace et qu'il peut augmenter de $25 \%$ l'autonomie d'un patient dément au stade modéré-sévère. Le nombre d'interactions requises entre l'aidant et le patient est également diminué lors de l'utilisation du guidage. Les auteurs n'ont pas noté d'effet d'apprentissage chez les participants pour la répétition de la tâche de lavage de mains sur 60 jours. Mihailidis et al. (2007) obtiennent sensiblement les mêmes résultats avec une étude de cas démontrant que le niveau de dépendance du participant envers l'aidant décroît avec l'introduction d'un guidage et augmente avec le retrait de celui-ci, toujours pour une tâche de lavage de mains. Ces auteurs évoquent cependant le fait que plusieurs interventions de guidage (entre $15 \%$ et $65 \%$ ) sont ignorées par le participant de sorte que le taux d'efficacité de ces dernières est variable. Cela suggère donc que le guidage verbal à lui seul pourrait ne 
pas fournir suffisamment d'information et d'assistance aux participants (Labelle \& Mihailidis, 2006). D'autres travaux se sont penchés sur l'étude de différentes méthodes de guidage et leur comparaison, toujours pour une population atteinte de démence. C'est entre autres le cas de Labelle et Mihailidis (2006) qui ont comparé chez 8 participants un guidage verbal et un guidage audiovisuel à des niveaux de base. Malgré le fait que les différences observées ne sont pas significatives entre le nombre d'étapes réussies d'une tâche de lavage de mains avec un guidage verbal et le nombre d'étapes réussies avec un guidage audiovisuel, les auteurs notent que certains participants ont bénéficié des guidages audiovisuels (plutôt qu'uniquement verbal). Ces derniers concluent donc que la modalité de guidage audiovisuel conserve un potentiel selon le contexte d'utilisation ainsi que le type d'usager, car cette modalité de guidage n'a pas diminué le niveau de performance ni engendré d'agitation particulière chez les participants. Les auteurs ont cependant noté une amélioration des performances entre les différentes phases de leur étude, pour la même activité de lavage de mains, qu'ils attribuent à un effet d'apprentissage ou encore de familiarisation avec la routine. Plus récemment, Mihailidis, Boger, Craig et Hoey (2008) ont étudié la dernière version de leur technologie (nommée $\mathrm{COACH}$ : Cognitive Orthosis for Assisting aCtvitities in the Home) avec 6 participants atteints de démence modérée à sévère. Le guidage verbal et le guidage audiovisuel ont été combinés et les résultats stipulent que le taux d'assistance fourni par les aidants a diminué de $66 \%$ en présence de la technologie. Les auteurs notent que les participants avec un degré de dépendance plus important ont été ceux qui ont bénéficié le plus des guidages informatisés. À l'inverse, la technologie n'a pas eu d'effet préjudiciable sur les 
participants plus indépendants. Les interventions de guidages pourraient donc être introduites à des stades précoces de la maladie sans effet néfaste supposé. Finalement, selon les auteurs, l'amélioration observée chez les participants de cette étude semble être le résultat de l'introduction du guidage informatisé plus que d'un effet d'apprentissage. Les effets observés sont cependant complexes et demandent à être étudiés davantage. En somme, les études mentionnées précédemment montrent que le guidage informatisé est efficace, cependant les résultats des études ne sont pas unanimes.

Une des limites des études réalisées dans le cadre de l'implantation de $\mathrm{COACH}$ (Labelle et Mihailidis, 2006; Mihailidis et al., 2004; Mihailidis et al., 2007; Mihailidis et al., 2008) est que les échantillons de participants sont relativement hétérogènes, car étant composés de personnes atteintes de plusieurs types de démences, chacune d'elles présentant des tableaux symptomatiques différents. De plus, le stade de la démence est déterminé par le score obtenu au Mini-Mental State Evaluation (MMSE), ce qui représente un indicateur plus ou moins fiable (Derouesné, 2006).

À ce jour, diverses méthodes de guidage ont été utilisées en recherche, mais plusieurs d'entre elles sans différenciation. Ainsi, le laboratoire utilisant la technologie COACH situé à l'Université de Toronto emploie un guidage vocal préenregistré, pouvant être combiné avec un guidage vidéo et comprenant différents niveaux de précision lors d'une tâche de lavage de mains (Mihailidis et al., 2008). Le niveau minimal d'assistance fournit des indications verbales générales quant aux étapes à compléter lorsque le 
participant commet une erreur dans la tâche. Les guidages mentionnent en premier lieu le nom du participant, effectuent ensuite un rappel quant à la tâche en cours et finalement donnent des indications générales (ex. : «John, vous êtes en train de vous laver les mains, séchez-vous les mains. »). Le niveau maximal d'assistance utilise à la fois un guidage verbal et visuel, utilise le nom du participant et fournit davantage de détails quant à l'action à effectuer (ex. : «John, vous êtes en train de vous laver les mains, utilisez la serviette bleue à votre droite »). Le laboratoire de l'Université de Sherbrooke (DOMUS) emploie quant à lui des guidages visuels (images, vidéos, lumières), vocaux ou sonores (Pigot et al., 2003), qui peuvent prendre la forme d'images ou de vidéos d'actions à effectuer, de lumières qui s'illuminent pour attirer l'attention du patient vers un endroit en particulier ou encore de voix et de sons guidant le patient. Les chercheurs du laboratoire DOMUS utilisent cependant ces types de guidage sans les différencier quant à leur propriété et les impacts potentiels sur le participant.

En raison du fait que le guidage s'avère partiellement efficace afin d'améliorer l'autonomie dans la réalisation d'une AVQ chez les patients déments (stades modéréssévères) (Mihailidis et al., 2004; Mihailidis et al., 2007, Mihailidis et al., 2008) et qu'une proportion importante de ces guidages est ignorée par les participants, il est pertinent de se questionner sur les divers facteurs pouvant contribuer à l'amélioration du taux d'utilisation du guidage par ces derniers. Selon Mihailidis et al. (2004), il semblerait que la réponse du participant au guidage soit reliée au degré de difficulté de l'activité en cours ainsi qu'au participant lui-même. Il existerait en ce sens des différences 
interindividuelles concernant la capacité du participant à répondre aux interventions de guidage et ce, en raison du fait que l'assistance technologique n'est pas adaptée au participant lui-même; en fonction notamment de la présentation spécifique de sa pathologie, du rythme différentiel d'évolution de la pathologie et de d'autres facteurs. De plus, Boger et al. (2006) affirment que le participant aurait davantage de chance de compléter une AVQ avec assistance s'il recevait un guidage qu'il est en mesure de comprendre et avec lequel il est familier. C'est pourquoi le guidage devrait être ajusté avec précision et répondre spécifiquement aux diverses particularités du patient (Serna, Pigot, \& Rialle, 2007). Nailos, Whitman et Maxwell (1994) ainsi que Van Tassel, Bouchard, Bouchard et Bouzouane (2010) abordent également le fait que l'efficacité du guidage pourrait être en lien avec le profil des participants (diagnostic, habiletés personnelles, déficits cognitifs) combiné aux caractéristiques de la tâche à effectuer (familiarité, complexité, nature de la tâche). Comme peu d'études ont été réalisées à ce jour concernant l'efficacité des guidages, et notamment avec une AVQ de nutrition, ce champ de recherche s'avère novateur.

\section{Le modèle PPH et l'évolution hétérogène de la MA:}

\section{impacts sur les mécanismes de guidage}

Selon le modèle de développement humain proposé par le PPH (Fougeyrollas et al., 1998), il existe une interaction complexe entre les facteurs personnels, les facteurs environnementaux et les habitudes de vie, interaction conduisant à la réalisation plus ou moins complète de ces habitudes de vie. Les facteurs personnels représentent les 
caractéristiques intrinsèques de la personne, telles que l'âge, le sexe, l'identité socioculturelle, les systèmes organiques, les aptitudes, etc. Dans la MA, les facteurs personnels tels que décrits par le modèle PPH diffèrent entre les individus, notamment en ce qui concerne les aptitudes et les différentes atteintes des systèmes organiques. En ce sens, les atteintes cognitives hétérogènes entre les patients pour un même stade d'évolution de la maladie (Joannette et al., 1995) influencent grandement le niveau de dépendance dans les AVQ (Jefferson et al., 2006). Les environnements prothétiques, et particulièrement le guidage, s'inscrivent donc potentiellement dans ce modèle en tant que facilitateur ( facteur environnemental qui favorise la réalisation des habitudes de vie lorsqu'il entre en interaction avec les facteurs personnels ») (Fougeyrollas et al., 1998) qui pourrait augmenter le niveau de participation aux AVQ de la personne atteinte. Cependant, les équipes de recherche travaillant actuellement à instaurer des facilitateurs environnementaux sous forme de guidage informatisé (Mihailidis et al., 2004; Pigot et al., 2003) ne prennent pas en compte de façon optimale les facteurs personnels des patients atteints de MA. C'est pourquoi cette étude tente d'explorer les atteintes cognitives en tant que facteurs personnels pouvant interférer avec le facilitateur environnemental, ici le guidage, et les habitudes de vie du patient atteint de MA.

\section{But de l'étude et objectifs de recherche}

Puisque la MA induit de nombreuses atteintes cognitives qui ont des répercussions négatives sur l'autonomie d'une personne ainsi que sur la capacité à réaliser des AVQ, des technologies d'assistance ont été utilisées afin de favoriser l'autonomie ainsi que le 
maintien à domicile. Cependant, en raison du fait qu'une proportion non négligeable des méthodes de guidage sont ignorées ou inutilisées par les participants avec MA, ces dernières doivent être adaptées à la clientèle cible, cela dans l'objectif d'améliorer l'autonomie lors de différentes AVQ. Ce projet de recherche se penche donc sur cette problématique de premier plan et a pour but de décrire l'efficacité de trois modalités de présentation du guidage (visuelle, verbale, audiovisuelle) et d'explorer les liens entre les atteintes cognitives et la réponse des participants aux différents types de guidage pour la réalisation d'une AVQ de préparation de repas (café et rôtie) et d'emballage d'un cadeau. La population étudiée est un groupe de patients atteints de MA en stade modéré.

\section{Questions de recherche}

1- Parmi les trois méthodes de guidage à l'étude, laquelle s'avère plus efficace chez les participants avec MA en stade modéré pour deux AVQ (préparation d'un café et d'une rôtie et emballage d'un cadeau)?

2- Est-il possible d'expliquer les facteurs relatifs d'efficacité du guidage par les atteintes neuropsychologiques des participants? 
Méthode 


\section{Participants}

La réalisation de ce projet de recherche implique le recrutement de participants, hommes et femmes, atteints de MA en stade modéré, soit les stades 3 à 5 (sur un maximum 7) de la Global Deterioration Scale (GDS) (Reisberg, Ferris, de Leon, \& Crook, 1982) et ayant moins de 90 ans. Ce critère d'âge a été établi en raison des normes des tests neuropsychologiques. Les participants sont recrutés au Centre de santé et de services sociaux Cléophas-Claveau de La Baie (Saguenay). Les critères d'exclusion du projet sont les suivants : mention dans le dossier médical de troubles psychiatriques majeurs ou de déficience sensorielle majeure non traités; et/ou d'antécédents de maladies ou de dommages au cerveau autres que la MA; et/ou de séquelles neurologiques conséquentes à des abus de drogues ou d'alcool.

\section{Instruments}

\section{Évaluation neuropsychologique}

Une évaluation neuropsychologique est effectuée afin de documenter les atteintes cognitives des participants, si aucune évaluation n'a été réalisée dans les trois derniers mois. Dans les cas où les participants ont été évalués dans la période prescrite, une autorisation de communiquer les résultats est demandée aux participants dans le but d'obtenir les résultats du neuropsychologue ayant effectué l'évaluation. Il est à noter que la certification d'éthique permet l'accès au dossier médical du patient et que le 
formulaire de consentement stipule clairement cette option. De plus, afin de rendre uniformes les évaluations effectuées par le Centre de santé et de services sociaux Cléophas-Claveau et celles effectuées par l'équipe de recherche, les mêmes instruments d'évaluation et les mêmes normes sont utilisés. L'évaluation neuropsychologique, si requise, est d'une durée prévue d'environ $1 \mathrm{~h} 30$ et porte sur les fonctions exécutives et instrumentales. Dans le cadre du protocole de recherche, les prochains tests sont administrés et utilisés aux fins d'analyses de type descriptif. Afin de déterminer les déficits neuropsychologiques des participants, les écarts à la moyenne sont calculés à l'aide de données normatives. Les scores $\mathrm{Z}$ de $-2,0$ écarts-types et moins sont considérés déficitaires (Lezak, Howieson, \& Loring, 2004; Meulemans \& Seron, 2000).

Global Deterioration Scale. La Global Deterioration Scale (GDS) (Reisberg et al. 1982) est une mesure clinique de la sévérité de la démence qui est largement utilisée dans le cas de la MA (McDougall, 1990). Elle permet une catégorisation détaillée en ce qui concerne l'évaluation de la démence à des stades sévères et est fréquemment utilisée dans la pratique clinique. L'utilisation de cette échelle requiert le jugement du clinicien (Hugonot-Diener, 2007) afin que ce dernier cote la sévérité de la démence sur une échelle de 1 (asymptomatique) à 7 (démence avancée) selon certaines caractéristiques cliniques et manifestations, par exemple, plaintes subjectives de troubles de la mémoire, difficulté attentionnelle, diminution de certaines capacités (Paul et al., 2002). Cette mesure est donc structurée de façon à mettre l'emphase sur les fonctions mnésiques ainsi que sur les capacités à effectuer les activités de la vie quotidienne (AVQ; par exemple, 
s'habiller, manger, se baigner, etc.) (Paul et al., 2002). Des études (Johansson \& Zarit, 1991; Overall, Scott, Rhoades, \& Lesser, 1990) ont démontré les qualités psychométriques de cette échelle, par exemple, une bonne fiabilité test-retest ainsi qu'une bonne validité pour ce qui est de la détermination des déficits fonctionnels et neuropsychologiques dans la MA (Paul et al., 2002).

Mini-Mental State Evaluation. Le Mini-Mental State Evaluation (MMSE; Folstein, Folstein, \& McHugh, 1975) est l'un des outils de dépistage les plus utilisés dans le domaine de la mesure générale des habiletés cognitives et ce, notamment en raison de sa facilité d'utilisation (Folstein, Folstein, McHugh, \& Fanjiang, 2001). Les 30 items du test (Lopez, Charter, Mostavi, Nibut, \& Smith, 2005) couvrent les capacités cognitives suivantes: mémoire, langage, capacités visuo-spatiales, calcul et attention (Schenk, Leuba, \& Büla, 2004). Un score inférieur ou égal à 23 détermine le point de coupure indiquant une altération du fonctionnement cognitif. Il est cependant nécessaire de tenir compte de l'âge et du niveau de scolarité dans l'interprétation des résultats. Par exemple, un même score au MMSE peut indiquer des degrés d'atteintes différents chez deux individus n'ayant pas le même degré de scolarité ou encore le même âge (Touchon \& Portet, 2002). Parallèlement, certaines critiques émanent d'études sur les qualités psychométriques de ce test, notamment la fiabilité qui est très variable selon le groupe de normalisation (Espino, Lichtenstein, Palmer, \& Hazuda, 2004; Hopp, Dixon, Backman, \& Grut, 1997; Lopez et al., 2005). Le MMSE est donc un outil de dépistage et il est recommandé de conserver une réserve lorsqu'il est question de l'utiliser dans 
l'évaluation la sévérité de la démence (Derouesné, 2006; Lopez et al., 2005). Les normes utilisées pour ce test sont celles de Crum, Anthony, Bassett et Folstein (1993).

Boston Naming Test. Le Boston Naming Test (BNT; Kaplan, Goodglass, \& Weintraub, 1983) est un test de dénomination verbale de 60 dessins simples soumis en ordre croissant de difficulté qui mesure les capacités d'accès lexical et d'élocution. Le temps alloué afin de nommer chaque item est de 20 secondes et le participant peut bénéficier d'indices sémantiques ou phonémiques s'il éprouve de la difficulté. Un score total est calculé à l'aide du nombre de bonnes réponses fournies spontanément additionné du nombre de bonnes réponses fournies après indice (Strauss, Sherman, \& Spreen, 2006). Lors de la passation du test, un critère d'arrêt est déterminé (après 6 échecs consécutifs) notamment afin de ne pas confronter le participant à une série d'échecs qui pourrait avoir des répercussions sur la suite de l'évaluation. La consistance interne du BNT se situe entre 0,78 et 0,96 (Fastenau, Denburg, \& Mauer, 1998; Storms, Saerens, \& De Deyn, 2004). De plus, les intervalles entre les passations font varier le degré de fiabilité du test. Lors d'intervalles courts, la fiabilité est élevée (Flanagan \& Jackson, 1997), alors que lors d'intervalles longs, la fiabilité peut varier de moyenne à élevée (Mitrushina \& Satz, 1995). Le BNT est également lié à d'autres mesures de langage telles que l'indice de compréhension verbale du WAIS-R (Axelrod, Ricker, \& Cherry, 1994). Les normes utilisées pour ce test sont les normes pour adultes publiées dans Spreen et Strauss (1998) et la variable utilisée est le nombre de réponses spontanées correctes. 
Examen des praxies. L'examen des praxies (Jason Brown modifié, source inconnue) comprend une liste de praxies idéomotrices que le participant doit exécuter avec la main gauche et la main droite. Il existe quatre parties soit les gestes intransitifs (10), les gestes transitifs (15), les gestes avec tout le corps (10) et la coordination bimanuelle (10). Ce test est considéré comme pathognomonique, les observations ainsi que le jugement clinique de l'évaluateur sont donc utilisés afin de déterminer l'atteinte des praxies chez le participant.

Protocole d'évaluation des gnosies visuelles. Le protocole d'évaluation des gnosies visuelles (PEGV, tiré du Protocole Montréal-Toulouse) est un outil permettant le dépistage de l'agnosie visuelle (Agniel, Joannette, Doyon, \& Duchein, 1992). Le test évalue entre autres les capacités de traitement des informations sensorielles (perception et discrimination de stimuli non verbaux) et de traitement sémantique fonctionnel ou catégoriel (identification de données visuelles). Le test contient quatre épreuves de désignation contenant chacune 10 items présentés sous forme de figures géométriques ou d'objets de la vie quotidienne. Deux épreuves concernent la discrimination visuelle (test des figures identiques, test des formes enchevêtrées) et les deux autres requièrent l'utilisation de processus sémantiques. Le temps alloué pour chaque item est de 30 ou 90 secondes. Pour chacune des épreuves, des scores limites sont identifiés indiquant un seuil pathologique. Les scores diffèrent pour chaque test (test des figures identiques : score $<8 / 10$, test des figures enchevêtrées : score $<30 / 36$, test d'appariement fonctionnel et test d'appariement catégoriel : score <9/10). Dans le cadre de ce projet de 
recherche, les deux premières épreuves (concernant la discrimination visuelle) sont administrées (omission des épreuves 3 et 4 ) afin de réduire le temps de passation et d'exclure les composantes faisant référence au langage et à l'aspect sémantique. Les scores limites énoncés ci-haut sont utilisés afin de déterminer l'atteinte des fonctions gnosiques chez les participants.

Buschke. Le Buschke (Buschke, 1984; modifié par Tuokko, \& Crockett, 1989) mesure les capacités d'apprentissage et la mémoire en modalité sensorielle visuelle et auditive verbale. Ce test comprend trois présentations de l'information (encodage), trois rappels immédiats et un rappel différé. Lors de l'administration de l'instrument, une feuille comprenant 12 objets est présentée au participant qui doit pointer et nommer l'objet correspondant à la catégorie sémantique donnée (ex. : un de ces objets est un meuble). Une fois la procédure de recherche des objets effectuée, le participant est invité à compter à rebours à partir de 100 sur une période de 60 secondes. Par la suite, le patient rappelle librement les items encodés et un indice sémantique est fourni (le même que lors de l'encodage) pour les items qui n'ont pas été rappelés. Si les indices sémantiques ne sont toujours pas efficaces afin de rappeler l'ensemble des mots, la feuille d'objets est représentée au participant qui effectue la procédure de recherche des mots qui n'ont pas été rappelés (O'Connel \& Tuokko, 2002). Ce test possède des caractéristiques intéressantes dans l'évaluation des troubles de mémoire. Premièrement, au point de vue neuropsychologique, il permet l'évaluation des capacités mnésiques ainsi que des informations encodées, mais non accessibles par le rappel libre (Buschke, 
1984). De plus, les indices rendent la tâche plus accessible aux patients ayant des atteintes cognitives en permettant de situer l'item dans un contexte, ce qui facilite le rappel (Tuokko \& Crockett, 1989). Ce test permet donc d'évaluer davantage de composantes (par une évaluation des capacités limites) qu'un simple test de rappel libre (Buschke, 1984). Finalement, les patients étant en mesure de répondre aux indices peuvent être plus enclins à bénéficier de ce genre de technique afin de performer dans différentes activités, ce qui pourrait avoir comme effet d'augmenter leur autonomie (O'Connell \& Tuokko, 2002). Le score total des trois rappels libres immédiats et du rappel libre différé sont les variables à l'étude. Les normes utilisées dans ce test sont celles publiées dans O'Connell \& Tuokko (2002).

Trail Making Test. Le Trail Making Test (TMT; Reitan, 1955) est un test mesurant la reconnaissance de lettres et de chiffres, le balayage visuel, la perception, le fonctionnement visuo-spatial, l'attention divisée, la vitesse motrice ainsi que la flexibilité cognitive (Anderson, 1994). Il requiert que le participant relie vingt-cinq chiffres en ordre croissant répartis sur une feuille (partie A) et vingt-cinq chiffres et lettres en alternance (partie B) le plus rapidement possible, sans commettre d'erreur. Le participant s'exerce tout d'abord à la tâche sur une feuille pratique. Cinq minutes (300 secondes) sont allouées afin de réaliser la partie B et il n'existe pas de limite de temps pour la partie A (Strauss et al., 2006). Les erreurs des participants sont corrigées à la mention de l'examinateur à mesure de leur survenue. La fiabilité interjuge du test est de 0,94 pour la partie A et de 0,90 pour la partie B (Fals-Stewart, 1991) et les deux parties 
du test corrèlent moyennement bien ensemble $(0,31$; Royan, Tombaugh, Rees, \& Francis, 2004). Les scores $Z$ des temps de réalisation de la tâche $A$ et de la tâche $B$ (qui comprennent la correction des erreurs) sont les données étudiées. Les normes utilisées pour la cotation de ce test sont celles de Tombaugh, Rees et McIntyre (1996).

Test du dessin de l'horloge. Le test du dessin de l'horloge (Mendez, Ala, \& Underwood, 1992), est une épreuve de dépistage de la démence qui évalue également les composantes visuo-constructive (motricité fine et graphisme) et les fonctions exécutives (planification/organisation) (Strauss et al., 2006). Il est demandé au participant de dessiner sur une feuille blanche une horloge avec tous les chiffres et les aiguilles indiquant l'heure à $11 \mathrm{~h} 10$. L'échelle d'interprétation qui sera utilisée dans ce test est celle de Mendez et al. (1992) qui décrit une série de critères afin d'obtenir un score total sur 20. Le test démontre une fiabilité interjuge élevée (Lezak, et al., 2004) ainsi qu’une fiabilité test-retest de 0,78 après 12 semaines (Mendez et al., 1992). Le test du dessin de l'horloge est moyennement à fortement corrélé avec le MMSE $(0,41$ à 0,80$)$ (Adunsky, Fleissig, Levenkrohn, Arad, \& Noy, 2002; Heinik, Solomesh, \& Beckman, 2004). Le score sur 20 est la variable utilisée qui sera normée à l'aide des données de Mendez et al., (1992).

Naturalistic Action Test

Le Naturalistic Action Test (NAT) mesure l'atteinte des AVQ chez les individus atteints de troubles cognitifs (Schwartz, Segal, Veramonti, Ferraro, \& Buxbaum, 2002). 
Ce test requiert l'exécution de trois tâches, soit la préparation d'un café (lait et sucre) et d'une rôtie (beurre et confiture), l'emballage d'un cadeau ainsi que la préparation d'une boîte à lunch et d'un sac d'école. Il est à noter que lors de la première tâche, seulement les objets nécessaires sont présents alors que pour les deux autres, le participant est en présence d'objets distracteurs visuellement ou sémantiquement similaires aux objets cibles. Dans le cadre de ce projet, les participants sont invités à réaliser les deux premières tâches du test (préparation d'un café et d'une rôtie et emballage d'un cadeau), la dernière tâche est donc omise en raison du fait qu'elle s'avère moins appropriée en fonction des caractéristiques des participants. Le NAT est un test dont les instructions, le placement des objets, les procédures d'intervention et les scores ont été validés empiriquement. $\Pi$ l sera utilisé dans le cadre de cette étude en tant que tâche expérimentale, car il possède de bonnes qualités psychométriques ainsi qu'une grande valeur écologique. En effet, le test est fiable au niveau de la cotation (fiabilité inter-juge, 95 à $98 \%)$ et la consistance interne $(0,79$ coefficient de Cronbach); la validité de construit (avec une batterie mesurant l'attention) ainsi que la validité concurrente (avec le Functional Independance Mesure, 0,36 à 0,71 et 0,51 à 0,72 ) sont également adéquates (Schwartz et al., 2002). Il a été démontré que les scores au NAT ne sont pas affectés par le niveau d'éducation, le genre ou encore les difficultés motrices telles que les hémiparésies. De plus, des corrélations significatives sont présentes entre les variables du NAT et les rapports des aidants concernant les AVQ (Schwartz et al., 2002). 


\section{Déroulement}

\section{Établissement de scripts d'activité de tâches tirées du NAT}

Afin de permettre l'identification des erreurs se produisant au cours d'une tâche et requérant l'introduction d'un guidage, des descriptions normatives d'une activité de nutrition (préparation d'un café avec lait et sucre et d'une rôtie avec beurre et confiture) et d'une activité d'emballage d'un cadeau, tirées du protocole du NAT (Schwartz et al., 2002), ont été obtenues. Laprise, Bouchard, Bouchard et Bouzouane (2010) ont réalisé une étude auprès de 20 participants contrôles adultes, âgés entre 18 et 77 ans (moyenne $36 \pm 17$ ), dans le but d'établir les étapes, transposées sous la forme de scripts normatifs, des tâches précédemment nommées. Quatre scripts ont été créés à la suite de l'expérimentation, soit un script d'exécution d'un café, un script d'exécution d'une rôtie, un script regroupant l'exécution d'un café et d'une rôtie simultanément ainsi qu'un script d'emballage d'un cadeau. Ces scripts décrivent l'ordre des étapes exécutées par des personnes sans altération cognitive connue de même que les étapes facultatives dont l'omission ne constitue pas une erreur et qui peuvent être effectuées à tout moment au courant de la tâche. En confrontant le comportement d'une personne avec MA à un script comportemental normatif lors de la réalisation de ces tâches, les erreurs sont détectées lorsque l'ordre des étapes requises n'est pas respecté ou encore lorsqu'une étape non facultative est omise (Laprise et al., 2010). L'intervention par guidage pourra dès lors être introduite. 


\section{Éthique et recrutement}

Ce projet de recherche a obtenu une certification d'éthique de l'Université du Québec à Chicoutimi (UQAC) et des protocoles d'ententes ont été établis entre le Centre de santé et de services sociaux Cléophas-Claveau et l'UQAC dans le but de procéder au recrutement des participants et d'obtenir les accès aux dossiers médicaux des participants retenus.

Tel que mentionné précédemment, le recrutement des participants est effectué par le Centre de santé et de services sociaux Cléophas-Claveau qui effectue le premier contact avec le participant potentiel. Le projet est sommairement expliqué aux participants admissibles à l'étude et l'accord est obtenu dans le but de transmettre les coordonnées du patient au responsable de la recherche.

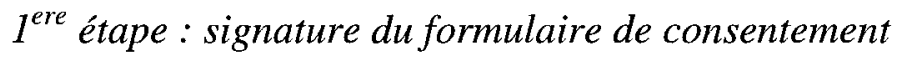

Suite à l'obtention des coordonnées du participant, une rencontre avec ce dernier est planifiée par un membre du projet de recherche. Lors de cette rencontre, le participant est en présence d'une tierce personne (membre de la famille ou responsable légal si le patient est sous tutelle ou sous curatelle) qui est en mesure de cosigner le formulaire de consentement (peu importe si la personne est déclarée apte ou inapte). La rencontre sert à expliquer le projet en détail, répondre aux questions et signer le formulaire de consentement. 
En ce qui a trait au formulaire de consentement, le membre de l'équipe de recherche explique de façon détaillée et dans un vocabulaire que le patient est en mesure de comprendre les modalités de la participation. Il s'assure également que le patient et le cosignataire comprennent bien l'ensemble du formulaire de consentement et ces derniers sont invités à poser des questions en ce sens. Finalement, une copie du formulaire de consentement est remise au patient (voir Appendice A).

$2^{e}$ étape : évaluation neuropsychologique

Une évaluation neuropsychologique est effectuée afin d'évaluer certaines composantes des fonctions instrumentales et exécutives, si aucune n'a été réalisée dans les trois derniers mois. La durée de cette rencontre est estimée entre 90 et 120 minutes. Le participant est donc rencontré afin d'effectuer les tests précédemment nommés et si l'évaluation a été effectuée à l'intérieur des trois mois, les données nécessaires aux analyses sont obtenues en ayant accès au dossier médical du patient. Dans les cas où le dossier du patient ne comprend pas l'ensemble des mesures requises, les évaluations manquantes sont effectuées.

$3^{e}$ étape : expérimentation

L'expérimentation comprend une rencontre pour chaque participant au cours de laquelle les trois temps de mesure sont effectués. Les participants sont appelés à se présenter à l'UQAC avec un membre de leur famille ou encore un responsable légal afin d'effectuer les tâches de préparation d'un café et d'une rôtie ainsi que d'emballage d'un 
cadeau tirées du protocole du NAT (Schwartz et al., 2002). Les participants sont également invités à remplir un questionnaire sociodémographique (voir Appendice B). La durée de la rencontre est estimée à 90 minutes.

Pour chacune des étapes des tâches, un guidage a été conçu à partir des scripts réalisés avec une population normale (Laprise et al., 2010). Notons que les scripts comprennent l'ensemble des étapes nécessaires à la réalisation d'une tâche. Un guidage a été conçu pour chaque étape selon les trois modalités de présentation (visuelle, verbale, audiovisuelle). Tous les guidages sont indépendants les uns des autres et décrivent une seule action à la fois. Ils ont été créés de façon à être le plus simple possible afin de tenter de ne pas surcharger par des informations inutiles la mémoire de travail du participant. Les méthodes de présentation du guidage sont celles retenues dans les études de Mihailidis et al. (2007) ainsi que dans Mihailidis et al. (2004), à l'exception de la voix de guidage. Aux fins de la présente expérimentation, le guidage verbal a été réalisé avec une voix féminine non familière comprenant les indications à effectuer afin de réaliser chacune des étapes. Dans le guidage visuel, des séquences vidéo comprenant les objets à utiliser ainsi que les actions à poser ont été réalisées et un son précédant le guidage est utilisé afin d'attirer l'attention du participant vers l'écran. Finalement, le guidage audiovisuel inclut à la fois la voix féminine et une vidéo comprenant les actions à effectuer et les objets à utiliser. Lors de l'expérimentation, le guidage est introduit au participant via un écran muni de haut-parleurs et un assistant de recherche déclenche manuellement le guidage lorsque le participant commet une erreur dans la tâche en cours 
à partir d'un programme comprenant des arbres décisionnels. Une erreur est détectée lorsqu'une étape nécessaire est omise ou encore lorsque l'ordre dans lequel les étapes nécessaires à la réalisation de la tâche doivent être effectuées n'est pas respecté. Il est à noter que le développement de la technologie informatique ainsi que des outils de reconnaissance d'activité permettront éventuellement que les erreurs soient identifiées par les systèmes d'intelligence ambiante et que les guidages soient introduits sans nécessité d'une intervention humaine.

Le déroulement des rencontres expérimentales s'effectue comme suit :

- En premier lieu, les participants sont accueillis et familiarisés avec l'environnement d'expérimentation. Un rappel est effectué en ce qui concerne le motif de leur présence.

- Les participants sont ensuite appelés à effectuer deux tâches (café/rôtie et emballage d'un cadeau) dans lesquelles un guidage informatisé est introduit (si nécessaire). Les instructions du NAT concernant la tâche à effectuer sont soumises au participant verbalement (voir Appendice C). Un guidage informatisé est introduit en cas d'erreur (ex. : mettre le café dans la tasse) ou après 30 secondes de comportement non productif (ex. : manipulation des objets sur la table sans but précis, inactivité) ou non progressif (ex. : comportement répétitif ou persévératif). Dans les cas où le participant ne répond pas à un premier guidage informatisé, un second est introduit après 30 secondes. Afin de maximiser le volume des données recueillies lors de l'expérimentation, les 
participants doivent compléter les tâches dans la mesure du possible, même s'ils ignorent ou ne répondent pas aux guidages qui leur sont fournis. Un second assistant de recherche est donc présent lors des séances d'expérimentation afin d'aider le participant à compléter l'étape en cours et ce, lorsque ce dernier ignore le guidage à deux reprises (un guidage introduit par 30 secondes) pour quelque raison que ce soit. Lorsque l'assistant de recherche est venu en aide au participant, ce dernier est invité à poursuivre la tâche. Dans les cas où le patient serait en mesure de compléter les étapes par lui-même ou encore qu'il répondrait aux guidages et que cela lui permettrait de compléter l'étape, le second assistant de recherche n'intervient pas. Cependant, dans les cas où le participant commente ou pose des questions à l'assistant de recherche, deux réponses peuvent être fournies : "Tout ce dont vous avez besoin est là. Faites de votre mieux. » ou «Je ne peux pas vous dire comment faire. Faites de votre mieux ». Le nombre de fois où l'assistant de recherche intervient avec le participant est pris en note et considéré comme une variable, ainsi que la nature de l'intervention. Des analyses pourront au besoin être faites quant à ces aspects, soit l'introduction d'une intervention humaine au cours d'une tâche avec guidages informatisés.

- La séance d'expérimentation est composée de trois blocs, soit les trois formes de guidage informatisés, comprenant chacun deux tâches (la préparation d'une rôtie et d'un café et l'emballage d'un cadeau). Les blocs correspondent donc au type de guidage qui est soumis, soit visuel, verbal ou audiovisuel. Ainsi, chacune des 
tâches est effectuée trois fois (en alternance) au cours de la séance d'expérimentation et le participant est soumis aux trois modalités de présentation du guidage au cours de cette même séance. Afin d'éviter les biais liés à l'ordre de présentation du guidage, l'échantillon est divisé en trois au hasard et l'ordre de présentation des blocs est alterné entre les participants. Par exemple, le bloc visuel occupera la première place pour le premier participant, la deuxième place pour le deuxième participant et la troisième place pour le troisième participant (voir Appendice D).

\section{Cotation des tâches}

Chacun des vidéos est visualisé par un assistant de recherche préalablement entraîné afin de procéder à la cotation de l'ordre des étapes de réalisation des tâches. Un formulaire comprenant la séquence des étapes réalisées, les erreurs commises, le moment d'introduction du guidage, le nombre de guidages émis ainsi que la réponse au guidage a été créé à cet effet. À titre d'exemple, voici une séquence partielle pouvant être réalisée par un participant pour la tâche café et rôtie :

1- prendre le beurrier

2- prendre le sac de pain

3- ouvrir le sac de pain

4- sortir une tranche de pain

5- prendre le beurrier

6- ouvrir le beurrier

7 - fermer le sac de pain

8- prendre le café

9- ouvrir le café, etc. 
Il est à noter que l'étape prendre le beurrier, qui est réalisée à deux reprises est cotée à l'étape 1 et 5 . De plus, l'étape fermer le sac de pain, qui constitue une étape facultative, est inclue dans la cotation de la séquence d'action. Finalement, puisque la tâche café et rôtie comprend deux sous-tâches (1- café, 2- rôtie), les étapes de chacune de ces soustâches sont comptabilisées conjointement afin d'obtenir un grand total.

\section{Analyses}

En raison de la difficulté à accéder à un large échantillon clinique, le présent protocole se veut une étude de cas multiples. Le devis expérimental est un plan intrasujet, chaque sujet est donc soumis à l'ensemble des niveaux (3) de la variable indépendante de guidage (verbal, visuel, audiovisuel). Les analyses se déroulent en deux étapes. En premier lieu, des analyses non paramétriques de comparaison de groupes (test $U$ de Mann-Whitney) sont réalisées entre l'échantillon clinique et l'échantillon de contrôle. Les variables à l'étude sont le nombre d'étapes réalisées et les temps d'exécution (secondes) des tâches. Pour chacune des tâches, soit la réalisation d'un café et d'une rôtie et l'emballage d'un cadeau, trois tests $U$ de Mann-Withney sont réalisés (en raison des trois temps de mesure des tâches) pour chacune des variables (correspondant au nombre d'étapes et au temps de réalisation). En second lieu, afin de répondre aux questions de recherche, des analyses descriptives sont effectuées afin de faire ressortir des liens entre les différents types de guidage et le nombre de réponses positives (question de recherche \#1) et les différents types de guidage, le nombre de 
réponses positives et les résultats d'évaluation neuropsychologique (question de recherche \#2).

Définition des variables de comparaison de groupes

Variable indépendante : type d'échantillon

- Échantillon de contrôle : données de l'étude de Laprise et al. (2010) impliquant 20 participants contrôles

- Échantillon clinique : données de la présente étude impliquant 3 participants atteints de MA en stade modéré

Variable dépendante :

- Nombre d'étapes de réalisation de la tâche : une étape constitue tout geste posé ou séquence d'action, qu'il soit ou non pertinent à l'évolution de la tâche en cours - Nombre d'erreurs commises au cours de la tâche : une erreur est commise lorsque l'ordre des étapes obligatoires à la réalisation de la tâche n'est pas respecté ou encore lorsqu'une étape obligatoire est omise

- Temps d'exécution de la tâche (en seconde) : le temps de réalisation de la tâche exclut pour les deux échantillons le temps nécessaire pour la présentation des consignes par l'assistant de recherche, mais inclut le temps de présentation des guidages pour l'échantillon clinique 
Définition des variables des analyses descriptives

Variable indépendante : Type de guidage

- Verbal : indications verbales de la séquence à effectuer.

- Visuel : vidéo indiquant les objets à utiliser ainsi que les actions à effectuer incluant un son précédant l'image à titre indicateur.

- Audiovisuel : vidéo des objets à utiliser et des actions à effectuer avec indications verbales simultanées.

Variable dépendante

- Nombre de réponses positives au guidage : un participant répond positivement au guidage si ce dernier lui permet de passer à la prochaine étape de la séquence d'action. Le score obtenu quant à cette variable est le nombre de réponses positives au guidage sur le nombre de présentations du guidage pour une même tâche, conduisant à la détermination d'un taux de réponse positive au guidage (ou taux d'efficacité) pour chaque méthode de guidage et pour chaque tâche. Ce taux sera transformé en pourcentage.

- Résultats d'évaluation neuropsychologique: les résultats de l'évaluation neuropsychologique seront pour chacun des participants décrits sous forme de déficits et de capacités préservées. 
Observations complémentaires

- Réaction du patient au guidage : les réactions des participants au guidage sont notées (par exemple : indifférence, peur, stupéfaction, rire, etc.) et analysées au besoin.

- Étape d'introduction du guidage dans la tâche : le moment d'introduction du guidage dans la tâche est pris en note et analysé au besoin. 
Résultats 


\section{Description de l'échantillon}

Six participants ont été recrutés sur. une période d'un an. Il est à noter que tous les participants ont été évalués en neuropsychologie au temps 1 par l'expérimentateur responsable. Aucune donnée neuropsychologique n'a donc été communiquée à l'équipe de recherche par le Centre Cléophas-Claveau exception faite du diagnostic médical, puisqu'un délai de plus de trois mois s'était écoulé pour les six participants entre la dernière évaluation neuropsychologique et la saisie des données de cette expérimentation. Par la suite, au temps 2, les participants ont été rencontrés pour l'expérimentation proprement dite. Des délais variant entre trois semaines et trois mois selon les participants se sont écoulés entre l'évaluation neuropsychologique du temps 1 et la participation au protocole expérimental au temps 2 (voir tableau 1).

Tableau 1

Temps écoulé entre l'évaluation neuropsychologique et l'expérimentation (jours)

\begin{tabular}{cc}
\hline Participant & Nombre de jours \\
\hline P1 & 23 \\
P2 & 23 \\
P3 & 87 \\
P4 & 49 \\
\hline
\end{tabular}

Six participants ont été recrutés dans le cadre de cette étude. Deux participants (P5 et P6) ont retiré leur consentement suite au premier temps d'expérimentation (évaluation neuropsychologique) et un autre participant (P4) a été retiré de l'échantillon pour les 
raisons suivantes: 1) des performances parfaites dans les tâches proposées (aucun guidage n'a été testé puisque ce dernier est introduit en cas d'erreur) et 2) l'atypie du profil neuropsychologique comparativement aux autres participants (performances normatives au Buschke). L'échantillon final est donc composé de trois participants, deux hommes (P1 et P2) et une femme (P3), âgés entre 70 et 81 ans (moyenne 74,3 $\pm 5,9$ ans) (voir tableau 2 résumant les données sociodémographiques). Les trois participants ont un diagnostic de MA dont deux avec composante vasculaire (P2 et P3) et le stade de la maladie selon la Global Deterioration Scale est estimé entre 4 et 5 (sur un maximum de

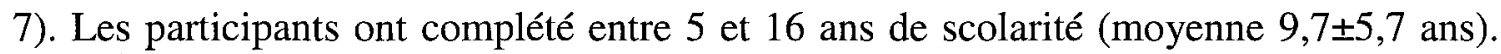
Tous demeurent avec un proche, soit un conjoint ou encore un enfant, et reçoivent de l'aide de celui-ci afin de compléter leurs activités de la vie quotidienne. Le revenu brut moyen (personnel ou familial) se situe entre 20000 \$ et 39999 \$. Tous les participants reçoivent un traitement pharmacologique composé de plusieurs médicaments. Le dossier médical du participant 1 (P1) fait état d'une dépression majeure pour laquelle il reçoit une médication au moment de l'expérimentation. Notons également que le participant 2 (P2) est le seul participant traité avec une médication ayant la propriété de retarder temporairement les symptômes liés à la maladie d'Alzheimer (voir tableau 3 décrivant des classes de médicaments prescrits pour chacun des participants). 
Tableau 2

Données sociodémographiques

\begin{tabular}{|c|c|c|c|c|c|c|c|c|}
\hline Participant & Âge & $\begin{array}{l}\text { Scolarité } \\
\text { (années) }\end{array}$ & $\begin{array}{l}\text { Diplôme } \\
\text { complété }\end{array}$ & $\begin{array}{l}\text { Occupation } \\
\text { antérieure }\end{array}$ & $\begin{array}{l}\text { Statut } \\
\text { civil }\end{array}$ & $\begin{array}{l}\text { Revenu } \\
\text { brut (\$) }\end{array}$ & Aidant & $\begin{array}{c}\text { Aide } \\
\text { aux } \\
\text { AVQ }\end{array}$ \\
\hline $\mathrm{P} 1$ & 70 & 16 & bacc & architecte & marié & $\begin{array}{l}20000- \\
39999\end{array}$ & conjointe & oui \\
\hline $\mathrm{P} 2$ & 81 & 5 & $\mathrm{n} / \mathrm{a}$ & ouvrier & veuf & $\begin{array}{c}20000- \\
39999\end{array}$ & fille & oui \\
\hline P3 & 72 & 8 & primaire & vendeuse & marié & $\begin{array}{c}20000- \\
39999\end{array}$ & conjoint & oui \\
\hline
\end{tabular}

Tableau 3

Médication

\begin{tabular}{lll}
\hline \multicolumn{1}{c}{ Participant 1 } & \multicolumn{1}{c}{ Participant 2 } & \multicolumn{1}{c}{ Participant 3 } \\
\hline Antidépresseur & Stimulants cognitifs & Hypotenseur \\
Anxiolytique & Hypoglycémiant & Hormone thyrö̈dienne \\
Hypocholestérolémiant & Hypocholestérolémiant & \\
Diurétique & Hypotenseur & \\
Antiviral & & \\
\hline
\end{tabular}

\section{Description des participants et évaluation neuropsychologique}

\section{Description de P1}

Le premier participant est un homme âgé de 70 ans, architecte retraité. Le diagnostic médical au dossier fait mention d'une maladie d'Alzheimer légère à modérée et d'une dépression majeure pour laquelle il est traité avec un antidépresseur ainsi qu'un anxiolytique. Monsieur demeure avec sa conjointe au domicile familial. L'évaluation neuropsychologique se déroule sans particularité et le stade de la maladie selon la GDS est évalué à 4 sur 7 (affaiblissement intellectuel modéré). Les résultats font ressortir des déficits dans les sphères suivantes: examen du statut mental (MMSE), orientation 
temporelle, mémoire épisodique verbale (court et long terme), accès lexical et flexibilité cognitive. Des difficultés sont également notées en ce qui concerne l'exécution de calculs mentaux (soustractions). Les capacités résiduelles se situent au niveau de l'orientation spatiale, des praxies, des gnosies ainsi que de la planification/organisation en tâche visuo-constructive. Les vitesses de traitement de l'information et de balayage visuel se situent également dans la norme, monsieur commet toutefois deux erreurs de séquence lors de cette tâche (voir tableau 4 pour la description des résultats de l'évaluation neuropsychologique).

\section{Description de P2}

Le deuxième participant est un homme âgé de 81 ans, retraité d'une compagnie d'aluminium pour laquelle il travaillait en tant qu'ouvrier. Le diagnostic médical au dossier fait mention d'une maladie d'Alzheimer probable avec composante vasculaire. Monsieur demeure avec sa fille qui se veut la principale aidante. L'évaluation neuropsychologique se déroule sans particularité et le stade de la maladie selon la GDS est évalué à 5 sur 7 (affaiblissement intellectuel modéré-grave). Les résultats font ressortir des déficits à l'examen du statut mental (MMSE), à l'orientation temporelle, en mémoire épisodique verbale (court et long terme), au niveau de l'accès lexical, de la planification/organisation en tâche visuo-constructive ainsi que de la flexibilité cognitive. Une apraxie idéomotrice ainsi qu'une agnosie visuelle sont observées, alors que des difficultés sont notées lors de tâches de calcul mental (soustraction) et de mémoire de 
travail. La vitesse de traitement de l'information et de balayage visuel est légèrement ralentie tandis que l'orientation spatiale est préservée.

\section{Description de P3}

Le troisième participant est une femme âgée de 72 ans, retraitée d'un commerce de vente de vêtements pour dame. Madame réside avec son conjoint dans un appartement faisant partie d'une maison bigénérationnelle. Le diagnostic médical au dossier est une maladie d'Alzheimer avec composante vasculaire. L'évaluation neuropsychologique se déroule sans particularité et le stade de la maladie selon la GDS est évalué à 4 sur 7 (affaiblissement intellectuel modéré). Les résultats font ressortir des déficits à l'examen du statut mental (MMSE), à l'orientation temporelle, en mémoire épisodique verbale (court et long terme), au niveau de l'accès lexical, de la vitesse de traitement de l'information et de balayage visuel ainsi que de la flexibilité cognitive. Madame présente en entrevue un manque du mot, une apraxie de type idéomotrice (qui pourrait être d'origine langagière) ainsi que des difficultés à l'exécution de calculs mentaux (soustraction). L'orientation spatiale est préservée et les capacités de planification/organisation se situent dans les normes en tâche visuo-constructive et en ce qui concerne les gnosies visuelles. 
Tableau 4

Résultats de l'évaluation neuropsychologique (score $\mathrm{Z}$ ou données nominales)

\begin{tabular}{|c|c|c|c|c|c|c|c|c|c|c|}
\hline & MMSE & $\begin{array}{l}\text { Orientation } \\
\text { temporelle }\end{array}$ & BNT & $\begin{array}{l}\text { Protocole } \\
\text { des } \\
\text { praxies }\end{array}$ & PEGV & $\begin{array}{c}\text { Buschke } \\
\text { total } \\
\text { rappel } \\
1-3\end{array}$ & $\begin{array}{c}\text { Buschke } \\
\text { rappel } \\
\text { libre } \\
\text { différé }\end{array}$ & $\begin{array}{l}\text { Trail } \\
\text { making } \\
\text { A }\end{array}$ & $\begin{array}{c}\text { Trail } \\
\text { making } \\
\text { B }\end{array}$ & $\begin{array}{c}\text { Dessin } \\
\text { de } \\
\text { l'horlo- } \\
\text { ge }\end{array}$ \\
\hline P1 & $-3,1$ & Déficitaire & $-4,8$ & $\begin{array}{c}\text { Non } \\
\text { déficitaire }\end{array}$ & $\begin{array}{c}\text { Non } \\
\text { déficitaire }\end{array}$ & $-5,5$ & $-6,4$ & $-0,3$ & & $-0,3$ \\
\hline $\mathrm{P} 2$ & $-3,2$ & $D$ & $-4,3$ & Déficitaire & Déficitaire & $-2,9$ & $-4,4$ & $-1,2$ & $\begin{array}{l}\text { Échec de } \\
\text { l'épreuve }\end{array}$ & $-8,0$ \\
\hline P3 & $-3,3$ & Déficitaire & $-4,8$ & Déficitaire & $\begin{array}{c}\text { Non } \\
\text { déficitaire }\end{array}$ & $-5,2$ & $-6,4$ & $-3,9$ & $\begin{array}{l}\text { Échec de } \\
\text { l'épreuve }\end{array}$ & $-0,3$ \\
\hline
\end{tabular}

MMSE : Mini-mental state evaluation

BNT : Boston naming test

PEGV: Protocole d'évaluation des gnosies visuelles

\section{Expérimentation : tâche café et rôtie}

Selon l'expérimentation réalisée auprès du groupe contrôle, la réalisation d'un café et d'une rôtie selon la consigne énoncée dans le NAT (Schwartz et al., 2002) (voir Appendice C) nécessite 26 étapes obligatoires en plus de 6 étapes facultatives, pour un total possible de 32 étapes. Les participants du groupe contrôle ont réalisé la tâche avec en moyenne 32,3 $( \pm 1,6)$ étapes et les trois participants avec MA ont réalisé la tâche avec en moyenne 36,7 $( \pm 4,5)$ étapes (voir tableau 5). La différence entre le nombre d'étapes du groupe contrôle et du groupe avec MA s'avère significative pour l'exécution de la tâche au temps $2(U=6,5, p<0,05)$ et $3(U=0, p<0,001)$, alors qu'aucune différence n'est mesurée au temps $1(U=29,5, p>0,05)$ (voir tableau 8). En ce qui concerne le nombre d'erreurs réalisées, les participants atteints de MA ont effectué en moyenne 1,9 $( \pm 0,8)$ erreurs par tâche (voir tableau 6) alors que les normaux ont réalisé la tâche sans en commettre (Laprise et al., 2010). Le temps de réalisation moyen chez les normaux est 
de $224( \pm 38)$ secondes (Laprise et al., 2010) alors que le temps de réalisation chez les participants atteints de MA est de $371( \pm 116)$ secondes (voir tableau 7). Le temps d'exécution moyen ainsi que la variance sont donc plus élevés chez les MA. Des différences significatives sont d'ailleurs mesurées entre les temps de réalisation du groupe contrôle et du groupe MA pour ce qui sont des temps 2 et 3 ( $U=0, p<0,001$ ). Aucune différence n'est cependant notée au temps $1(U=19, p>0,05)$ (voir tableau 8).

L'effet d'apprentissage au cours de la tâche peut quant à lui être évalué en fonction de deux paramètres, soit le nombre d'étapes réalisées au cours des trois essais de même que le nombre d'erreurs commises. En comparant le nombre d'étapes effectuées au cours de chacune des passations pour un même participant, aucun effet d'apprentissage dû à l'exécution répétée des tâches (à trois reprises) n'est observé pour la tâche café et rôtie. En effet, le nombre d'étapes augmente au cours des essais ou demeure sensiblement le même. En ce qui concerne le nombre d'erreurs commises, aucune diminution du nombre d'erreurs n'est observée auprès des participants 1 et 2 . Cependant, le participant 3 démontre une diminution d'erreur entre le deuxième et le troisième essai, ce qui pourrait possiblement constituer un effet d'apprentissage. 


\section{Tableau 5}

Nombre d'étapes tâche café et rôtie

\begin{tabular}{ccccccc}
\hline \multirow{2}{*}{ Participant } & \multicolumn{2}{c}{ T1 } & \multicolumn{2}{c}{ T2 } & \multicolumn{2}{c}{ T3 } \\
\cline { 2 - 7 } & Étapes & Score Z & Étapes & Score Z & Étapes & Score Z \\
\hline P1 & 29 & $-2,1$ & 33 & 0,4 & 41 & 5,4 \\
P2 & 32 & $-0,2$ & 35 & 1,7 & 41 & 5,4 \\
P3 & 39 & 4,2 & 41 & 5,4 & 39 & 4,2 \\
\hline
\end{tabular}

Nombre moyen d'étapes chez les normaux : $32,3( \pm 1,6)$

Tableau 6

Nombre d'erreurs tâche café et rôtie

\begin{tabular}{ccccc}
\hline Participant & T1 & T2 & T3 & Total \\
\hline P1 & 1 & 2 & 2 & 5 \\
P2 & 1 & 2 & 2 & 5 \\
P3 & 3 & 3 & 1 & 7 \\
\hline
\end{tabular}

Nombre moyen d'erreurs chez les normaux : $0( \pm 0)$

Tableau 7

Temps de réalisation tâche café et rôtie (secondes)

\begin{tabular}{ccccccc}
\hline \multirow{2}{*}{ Participant } & \multicolumn{2}{c}{ T1 } & \multicolumn{2}{c}{ T2 } & \multicolumn{2}{c}{ T3 } \\
\cline { 2 - 7 } & Temps & Score Z & Temps & Score Z & Temps & Score Z \\
\hline P1 & 170 & $-1,4$ & 320 & 2,5 & 350 & 3,3 \\
P2 & 522 & 7,8 & 386 & 4,3 & 360 & 3,6 \\
P3 & 561 & 8,8 & 361 & 3,6 & 306 & 2,2 \\
\hline
\end{tabular}

Temps de réalisation moyen chez les normaux : $224( \pm 38)$ secondes 
Tableau 8

Comparaison du groupe contrôle et du groupe clinique tâche café et rôtie

\begin{tabular}{llc}
\hline \multicolumn{1}{c}{ Variable } & \multicolumn{1}{c}{ Test de Mann-Whitney } & Signification \\
\hline Nombre d'étapes T1 & $\mathrm{U}=29,5, \mathrm{Z}=-0,048$ & $\mathrm{~ns}$ \\
Nombre d'étapes T2 & $\mathrm{U}=6,5, \mathrm{Z}=-2,22$ & 0,026 \\
Nombre d'étapes T3 & $\mathrm{U}=0, \mathrm{Z}=-2,83$ & 0,001 \\
\hline Temps de réalisation T1 & $\mathrm{U}=19, \mathrm{Z}=-1,00$ & $\mathrm{~ns}$ \\
Temps de réalisation T2 & $\mathrm{U}=0, \mathrm{Z}=-2,74$ & 0,001 \\
Temps de réalisation T3 & $\mathrm{U}=0, \mathrm{Z}=-2,74$ & 0,001 \\
\hline
\end{tabular}

Expérimentation : tâche cadeau

L'expérimentation auprès de normaux démontre que la tâche d'emballage d'un cadeau se réalise en 18 étapes obligatoires en plus de 3 étapes facultatives, pour un total possible de 21 étapes. Les participants de 1'échantillon de contrôle ont réalisé la tâche avec en moyenne 19,5 $( \pm 1,8)$ étapes et les trois participants MA ont réalisé la tâche avec en moyenne 18,6 $( \pm 2,1)$ étapes (voir tableau 9). Il existe une différence significative entre le nombre d'étapes pour les deux groupes aux temps 2 et $3(U=0, p<0,001)$, alors que la différence au temps 1 n'est pas significative $(U=24,5, p>0,05)$ (voir tableau 12). Le nombre d'erreurs moyen par essai chez les participants atteints de MA est de 1,6 ( $\pm 1,2)$ (voir tableau 10) alors que les normaux réalisent la tâche sans commettre d'erreur (Laprise et al., 2010). Chez un échantillon contrôle, le temps moyen d'exécution de la tâche d'emballage d'un cadeau est de $336( \pm 130)$ secondes (Laprise et al., 2010) alors qu'il est de $476( \pm 130)$ secondes pour l'échantillon clinique (voir tableau 11). Tel qu'observé dans la tâche de café et rôtie, le temps d'exécution moyen ainsi que 
la variation pour la tâche d'emballage d'un cadeau sont légèrement plus élevés chez l'échantillon atteint de MA, cette différence est significative au temps $1(U=2, p<$ $0,005)$, mais ne l'est pas aux temps $2(U=21, p>0,05)$ et $3(U=18, p>0,05)$ (voir tableau 12).

En ce qui concerne l'effet d'apprentissage, une diminution du nombre d'étapes au cours des essais est observée chez P1. Le nombre d'étapes réalisées par P2 au temps 2 doit quant à lui être interprété avec réserve en raison de certaines problématiques ayant eu lieu lors de l'expérimentation (ex. le jouet à emballer était déjà placé dans la boîte, trois étapes de moins ont donc été nécessaires lors de l'expérimentation soit prendre le jouet, emballer le jouet avec du papier de soie et mettre le jouet dans la boîte). Chez P3, le nombre d'étapes requises pour accomplir la tâche subit pour sa part une légère augmentation entre le $2^{\mathrm{e}}$ et le $3^{\mathrm{e}}$ essai, ce qui exclut la possibilité d'un effet d'apprentissage. Concernant le nombre d'erreurs, une augmentation est observée chez P1 entre le $1^{\text {er }}$ et le $2^{\mathrm{e}}$ essai, alors qu'une diminution est observée au cours des essais chez P2 et P3. Les performances des deux derniers participants pourraient indiquer un effet d'apprentissage. 


\section{Tableau 9}

Nombre d'étapes tâche cadeau

\begin{tabular}{cccccccc}
\hline \multirow{2}{*}{ Participant } & \multicolumn{2}{c}{ T1 } & \multicolumn{2}{c}{ T2 } & \multicolumn{2}{c}{ T3 } \\
\cline { 2 - 7 } & Étapes & Score Z & Étapes & Score Z & Étapes & Score Z \\
\hline P1 & 20 & 0,3 & 18 & $-0,8$ & 18 & $-0,8$ \\
P2 & 20 & 0,3 & 15 & $-2,5$ & 16 & $-1,9$ \\
P3 & 19 & $-0,3$ & 19 & $-0,3$ & 22 & 1,4 \\
\hline
\end{tabular}

Nombre moyen d'étapes chez les normaux : 19,5 $( \pm 1,8)$

Tableau 10

Nombre d'erreurs tâche cadeau

\begin{tabular}{ccccc}
\hline Participant & T1 & T2 & T3 & Total \\
\hline P1 & 1 & 2 & 2 & 5 \\
P2 & 4 & 2 & 2 & 8 \\
P3 & 1 & 0 & 0 & 1 \\
\hline
\end{tabular}

Nombre moyen d'erreurs chez les normaux : $0( \pm 0)$

\section{Tableau 11}

Temps de réalisation tâche cadeau (secondes)

\begin{tabular}{ccccccc}
\hline \multirow{2}{*}{ Participant } & \multicolumn{2}{c}{ T1 } & \multicolumn{2}{c}{ T2 } & \multicolumn{2}{c}{ T3 } \\
\cline { 2 - 7 } & Temps & Score Z & Temps & Score Z & Temps & Score Z \\
\hline P1 & 711 & 2,9 & 320 & $-0,1$ & 290 & $-0,4$ \\
P2 & 518 & 1,4 & 440 & 0,8 & 448 & 0,9 \\
P3 & 526 & 1,5 & 441 & 0,8 & 591 & 2,0 \\
\hline
\end{tabular}

Temps de réalisation moyen chez les normaux : $336( \pm 130)$ secondes 
Tableau 12

Comparaison du groupe contrôle et du groupe clinique tâche cadeau

\begin{tabular}{llc}
\hline \multicolumn{1}{c}{ Variable } & \multicolumn{1}{c}{ Test de Mann-Whitney } & Signification \\
\hline Nombre d'étapes T1 & $\mathrm{U}=24,5, \mathrm{Z}=-0,52$ & $\mathrm{~ns}$ \\
Nombre d'étapes T2 & $\mathrm{U}=0, \mathrm{Z}=-2,83$ & 0,001 \\
Nombre d'étapes T3 & $\mathrm{U}=0, \mathrm{Z}=-2,83$ & 0,001 \\
\hline Temps de réalisation T1 & $\mathrm{U}=2, \mathrm{Z}=-2,56$ & 0,005 \\
Temps de réalisation T2 & $\mathrm{U}=21, \mathrm{Z}=-0,82$ & $\mathrm{~ns}$ \\
Temps de réalisation T3 & $\mathrm{U}=18, \mathrm{Z}=-1,10$ & $\mathrm{~ns}$ \\
\hline
\end{tabular}

Questions de recherche \#1 : Parmi les trois méthodes de guidage à l'étude, laquelle

s'avère plus efficace chez les participants avec MA en stade modéré pour deux AVQ

(préparation d'un café et d'une rôtie et emballage d'un cadeau)?

\section{Participant 1}

Lors de l'exécution de la tâche café et rôtie, P1 a effectué 5 erreurs au cours des trois essais (moyenne 1,7 $\pm 0,6$ ). Les étapes au cours desquelles P1 a commis des erreurs sont les suivantes (pour les trois essais) : prendre le café, saisir la bouilloire, mettre le pain dans le grille-pain (2 erreurs) et prendre la confiture. Pour ces 5 erreurs, le guidage a été introduit à 8 reprises (un guidage peut être introduit jusqu'à deux fois si le participant ne répond pas à la première présentation de guidage) et 2 se sont avérés efficaces. Les modalités pour lesquelles le guidage a été efficace pour ce participant sont le guidage verbal à $33 \%$ ( 1 guidage efficace sur 3 présentations) ainsi que le guidage audiovisuel également à $33 \%$ (1 guidage efficace sur 3 présentations). Le participant a mentionné à une reprise son incompréhension suite à la présentation d'un guidage audiovisuel et a 
ignoré (aucune réaction) les autres guidages (5 guidages sur 8). L'Appendice E illustre les étapes d'introduction des différents guidages pour la tâche café et rôtie.

En ce qui concerne la tâche d'emballage d'un cadeau, P1 a effectué 5 erreurs au cours

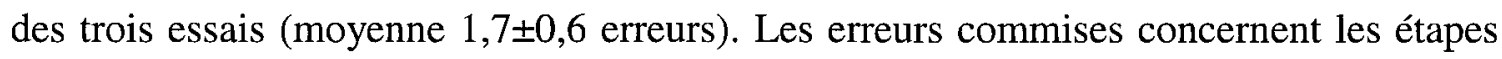
suivantes (pour les trois essais) : dérouler le papier d'emballage (2 erreurs), prendre le sac de chou (2 erreurs) et enlever le papier protecteur sur le chou. Pour ces 5 erreurs, le guidage a été introduit à 7 reprises et 3 se sont avérés efficaces. Un guidage sur 3 présentations $(33 \%)$ s'est avéré efficace en modalité verbale et 2 guidages sur 2 présentations (100\%) ont été efficaces en modalité audiovisuelle. Monsieur a ignoré (aucune réaction) les autres guidages présentés (4 guidages sur 7). Il apparaît important de mentionner quelques comportements émis par P1 au cours des essais de réalisation de cette tâche. En premier lieu, le participant s'efforçait de chercher sur la table un papier d'emballage identique à celui présenté sur l'exemple du NAT (un exemple de la tâche complétée est représenté par un dessin en noir et blanc), alors que le papier d'emballage disponible était de couleur jaune et comprenait des motifs. De plus, le participant persévérait à apposer des choux sur le cadeau afin, selon ses dires, de tenter de reproduire celui illustré sur l'exemple. Des interventions ont donc été nécessaires afin d'expliquer au participant qu'il devait se fier à l'image à titre indicatif et que son cadeau ne devait pas être identique en tout point. L'Appendice F illustre les étapes d'introduction des différents guidages pour la tâche d'emballage d'un cadeau. 
En regroupant les résultats des deux tâches réalisées lors de l'expérimentation (6 essais), P1 a répondu efficacement à $33 \%$ (pourcentage obtenu par le nombre de réussites/nombre d'essais) des guidages proposés, $67 \%$ des guidages ont donc été ignorés. Le guidage visuel s'est avéré complètement inefficace, alors que les guidages verbal et audiovisuel se sont avérés efficaces 2 fois sur 6 et 3 fois sur 5 respectivement (voir tableau 11).

\section{Participant 2}

$\mathrm{Au}$ cours des trois essais de réalisation d'un café et d'une rôtie, P2 a effectué 5

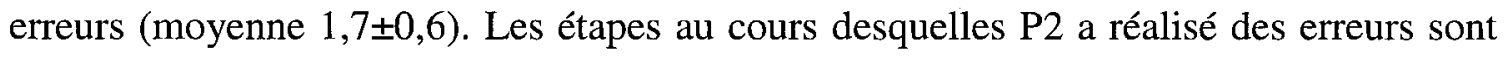
les suivantes (pour les trois essais) : prendre le sac de pain, prendre la confiture (3 erreurs) et prendre le sucrier (voir Appendice E). Pour ces 5 erreurs, le guidage a été introduit à 7 reprises et 2 guidages sur 2 présentations (100\%) se sont avérés efficaces, seulement en modalité verbale. Monsieur a émis à une reprise une réaction de surprise en entendant la voix du guidage verbal et semblait se questionner quant à la provenance de cette voix. Cette intervention de guidage s'est malgré tout avérée efficace. Monsieur a ignoré (aucune réaction) les autres présentations de guidages (5 guidages sur 7).

Pour ce qui est de la tâche d'emballage d'un cadeau, P2 a effectué 8 erreurs (moyenne 2,7 71,2 ) au cours des trois essais. Les étapes au cours desquelles monsieur a commis des erreurs sont les suivantes (pour les trois essais) : prendre le jouet (2 erreurs), plier le papier à la deuxième extrémité de la boîte (2 erreurs), coller le papier à la 
deuxième extrémité de la boîte et prendre le sac de chou ( 3 erreurs) (voir Appendice F). Pour les 8 erreurs commises, le guidage a été introduit à 16 reprises. Parmi l'ensemble des présentations de guidage, 1 seul guidage sur 4 présentations (25\%) s'est avéré efficace en modalité audiovisuelle. Outre les fois où monsieur a ignoré (aucune réaction) les présentations de guidage (14 guidages sur 16), monsieur a verbalisé à une reprise son incompréhension lors d'une présentation de guidage en modalité audiovisuelle.

En regroupant les deux tâches réalisées lors de l'expérimentation (6 essais), P2 a répondu efficacement à $13 \%$ des guidages, $87 \%$ des guidages ont donc été ignorés. Le guidage visuel s'est avéré complètement inefficace, alors que le guidage verbal a été efficace 2 fois sur 2 et le guidage audiovisuel 1 fois sur 4 (voir tableau 11).

\section{Participant 3}

$\mathrm{Au}$ cours des différents essais de réalisation d'un café et d'une rôtie, $\mathrm{P} 3$ a réalisé 7 erreurs (moyenne 2,3 $\pm 1,2$ ). Les étapes au cours desquelles les erreurs ont été réalisées sont les suivantes (pour les trois essais) : mettre le pain dans le grille-pain, prendre la confiture ( 3 erreurs), prendre le lait, prendre le sucrier et sortir une tranche de pain (voir Appendice E). Pour les 7 erreurs commises, 9 guidages ont été introduits. Madame a répondu positivement à 3 des 9 guidages, ces guidages étant soit de modalité verbale ou encore audiovisuelle. Cent pour cent des guidages ont été efficaces dans ces deux modalités ( 2 guidages sur 2 présentations en modalité verbale et 1 guidage sur 1 
présentation en modalité audiovisuelle). Madame a ignoré (aucune réaction) 6 des 9 guidages, tous en modalité visuelle.

$\mathrm{Au}$ cours de la tâche d'emballage d'un cadeau, P3 a réalisé 1 erreur (moyenne $0,3 \pm 0,6$ ) à l'étape prendre le jouet (voir appendice $F$ ). Toutefois, en raison d'une problématique informatique, le logiciel n'a pas émis de guidage en modalité visuelle. Les trois essais ont donc été réalisés sans introduction de guidage, car madame n'a pas commis d'erreur lors des essais en modalité verbale et audiovisuelle.

En tenant compte de la tâche café et rôtie (3 essais), P3 a répondu efficacement à $40 \%$ des guidages présentés, $60 \%$ des guidages ont donc été ignorés. Le guidage visuel s'est avéré inefficace alors que les guidages verbal et audiovisuel ont été efficaces 2 fois sur 2 et 1 fois sur 1 respectivement (voir tableau 11).

\section{Résumé de l'efficacité des guidages}

En somme, les guidages verbaux et audiovisuels se sont avérés partiellement efficaces pour les participants alors que le guidage visuel s'est avéré complètement inefficace. En combinant les résultats obtenus aux deux tâches, le guidage verbal a été efficace à $33 \%$ pour $\mathrm{P} 1,20 \%$ pour $\mathrm{P} 2$ et à $100 \%$ pour $\mathrm{P} 3$, pour une moyenne cumulative de $51 \%$ (49\% des guidages en moyenne ont été ignorés). Le guidage audiovisuel a été efficace à $60 \%$ pour $\mathrm{P} 1,14 \%$ pour $\mathrm{P} 2$ et à $100 \%$ pour $\mathrm{P} 3$, pour une moyenne cumulative de $58 \%$ (42\% des guidages en moyenne ont été ignorés). Le 
guidage audiovisuel semble donc plus efficace que les deux autres modalités, cependant les différents taux d'efficacité varient de façon importante selon les participants.

Tableau 13

Efficacité des guidages selon les participants

\begin{tabular}{|c|c|c|c|}
\hline Participant & Tâche café et rôtie & Tâche cadeau & $\begin{array}{c}\text { Efficacité } \\
(\%)\end{array}$ \\
\hline \multicolumn{4}{|c|}{ Guidage visuel } \\
\hline$\overline{P 1}$ & $0 / 2$ & $0 / 2$ & 0 \\
\hline $\mathrm{P} 2$ & $0 / 2$ & $0 / 4$ & 0 \\
\hline P3 & $0 / 6$ & Nil & 0 \\
\hline Moyenne (\%) & & & 0 \\
\hline \multicolumn{4}{|c|}{ Guidage verbal } \\
\hline P1 & $1 / 3$ & $1 / 3$ & 33 \\
\hline $\mathrm{P} 2$ & $2 / 2$ & $0 / 8$ & 20 \\
\hline P3 & $2 / 2$ & Nil & 100 \\
\hline Moyenne (\%) & & & 51 \\
\hline \multicolumn{4}{|c|}{ Guidage audiovisuel } \\
\hline P1 & $1 / 3$ & $2 / 2$ & 60 \\
\hline $\mathrm{P} 2$ & $0 / 3$ & $1 / 4$ & 14 \\
\hline P3 & $1 / 1$ & Nil & 100 \\
\hline Moyenne (\%) & & & 58 \\
\hline
\end{tabular}

Question de recherche \#2 : Est-il possible d'expliquer les principes relatifs d'efficacité du guidage par les atteintes neuropsychologiques des participants?

Sur le plan de l'évaluation neuropsychologique, les participants démontrent, d'une part, des profils communs d'atteintes et, d'autre part, certaines spécificités. Les atteintes communes chez tous les participants se situent au niveau de l'examen du statut mental (MMSE), de l'orientation temporelle, de la mémoire épisodique verbale à court et long terme, de l'accès lexical ainsi que du fonctionnement exécutif (flexibilité cognitive). Les 
atteintes spécifiques pour chacun des participants sont décrites dans les paragraphes suivants.

\section{Participant 1}

L'évaluation neuropsychologique ne fait pas ressortir pour P1 d'autres déficits outre ceux communs chez tous les participants. Les sphères les plus atteintes (en fonction des scores Z) sont la mémoire épisodique verbale à court et long terme de même que la flexibilité cognitive. Les capacités préservées se situent sur le plan de l'orientation spatiale, des praxies, des gnosies, de la vitesse de traitement de l'information et de balayage visuel de même que des capacités de planification/organisation lors d'une tâche visuo-constructive. L'efficacité du guidage pour ce participant est partielle. En effet, le taux d'efficacité est de $33 \%$ en modalité verbale et de $60 \%$ en modalité audiovisuelle. Pour ce participant, le guidage audiovisuel est donc plus efficace. Comme les épreuves visuelles font partie des capacités préservées (gnosie, balayage visuel, planification/organisation en tâche visuo-constructive), il semble que la modalité audiovisuelle puisse être celle qui soit la plus efficace pour ce participant. En effet, les tâches de mémoire épisodique de même que de flexibilité cognitive, bien que ne faisant pas uniquement appel à la composante auditive, requièrent la capacité d'effectuer une boucle articulatoire auditive mentale de l'information afin de réaliser la tâche (ex. : les items à rappeler du test de mémoire ainsi que l'alphabet et les séquences de chiffres pour la tâche de flexibilité cognitive), élément qui semble être affecté compte tenu du profil du participant. Cette boucle articulatoire est nécessaire à l'utilisation des guidages 
verbaux et c'est pourquoi il est supposé, pour ce participant, que l'addition de la composante visuelle à la composante verbale s'avère aidante.

\section{Participant 2}

Le participant 2 démontre des atteintes dans l'ensemble des sphères de l'évaluation neuropsychologique, excepté à l'orientation spatiale ainsi qu'en vitesse de traitement de l'information et de balayage visuel. Les capacités résiduelles, toujours en fonction de l'évaluation neuropsychologique, sont donc restreintes chez ce participant. Les sphères les plus déficitaires se situent au niveau des fonctions exécutives, soit la flexibilité cognitive ainsi que les capacités de planification/organisation en tâche visuoconstructive. Notons que parmi les participants de l'échantillon, P2 démontre le profil d'atteintes le plus important. De plus, le stade de la maladie (stade 5 selon la GDS) est plus élevé que P1 et P3. Les taux d'efficacité des guidages pour le deuxième participant sont de $20 \%$ en modalité verbale et de $14 \%$ en modalité audiovisuelle, le guidage verbal serait donc plus efficace. Ces valeurs représentent cependant les taux les plus faibles de l'échantillon. Les atteintes sur le plan de la perception et de l'analyse visuelle, notamment au niveau des gnosies et de la planification/organisation en tâche visuoconstructive, peuvent rendre plus difficile pour ce participant l'analyse de la composante visuelle du guidage, ce qui pourrait expliquer que le guidage visuel soit complètement inefficace et que le guidage audiovisuel soit peu efficace. La sévérité des atteintes neuropsychologiques pour ce participant n'est pas non plus à négliger dans la compréhension de l'efficacité des guidages. 


\section{Participant 3}

Outre les déficits communs à l'ensemble des participants, P3 démontre des performances sous la norme attendue en ce qui concerne les praxies idéomotrices. Les déficits les plus importants concernent la mémoire épisodique verbale à court et long terme ainsi que la flexibilité cognitive. Les capacités préservées se situent donc au niveau de l'orientation spatiale, des gnosies, de la vitesse de traitement de l'information et de balayage visuel ainsi que des capacités de planification/organisation en tâche visuo-constructive. Les taux d'efficacité du guidage pour le troisième participant, uniquement pour la tâche café et rôtie (car madame avait effectué une seule erreur en tâche d'emballage d'un cadeau et le guidage n'avait pas fonctionné à ce moment) sont de $100 \%$ pour les modalités verbale et audiovisuelle. Les deux types de guidages s'avèrent donc également efficaces. Ces taux représentent les plus hautes valeurs de l'échantillon et le participant 3 est le seul pour qui les guidages verbal et audiovisuel se sont avérés complètement efficaces en tâche café et rôtie. Pour ce participant, il semble donc que la composante visuelle soit aussi efficace que la composante verbale. L'évaluation neuropsychologique révèle cependant que les capacités préservées concernent majoritairement les capacités visuelles (gnosies, balayage visuel, planification-organisation en tâche visuo-constructive), ce qui devrait favoriser le guidage audiovisuel par rapport au guidage uniquement verbal. Finalement, le nombre de guidages présentés dans les modalités verbale et audiovisuelle pour les deux types de tâches est inférieur pour $\mathrm{P} 3$ comparativement à $\mathrm{P} 1$ et $\mathrm{P} 2$ (seulement 3 guidages ont été émis contre 11 pour P1 et 17 pour P2). Moins d'erreurs ont donc été commises et le 
faible nombre de présentations de guidage aurait pu influencer les taux de réponse à la hausse. 
Discussion 
Cette étude exploratoire à cas multiples réalisée auprès d'un échantillon composé de trois participants atteints de $\mathrm{MA}$ en stade modéré vise dans un premier temps à déterminer l'efficacité relative de trois types de guidage (visuel, verbal et audiovisuel) au cours de deux tâches, soit la réalisation d'un café et d'une rôtie d'une part et l'emballage d'un cadeau d'autre part. Dans un deuxième temps, l'étude cherche à explorer l'influence du profil neuropsychologique du participant sur l'efficacité du guidage.

\section{Comparaison des performances : groupe contrôle et groupe clinique}

\section{Expérimentation : tâche café et rôtie}

L'analyse descriptive des résultats permet de constater que le nombre moyen d'étapes effectuées lors de la réalisation d'un café et d'une rôtie est plus élevé chez les participants atteints de MA que chez les normaux. Les analyses statistiques sont cependant partagées, une différence significative étant mesurée entre ces deux groupes aux temps 2 et 3 mais non au temps 1. Dans la littérature, il est connu que les atteintes cognitives engendrées par la MA entraînent une difficulté lors de la réalisation d'AVQ (Derouesné, 2006), ce qui peut augmenter le nombre d'étapes de réalisation des tâches. De plus, les observations expérimentales indiquent que les participants ont émis plusieurs comportements répétitifs ou persévératifs au cours des tâches, élément pouvant également influencer le nombre d'étapes réalisées. Les pistes suivantes peuvent justifier 
la présence de comportements répétitifs ou persévératifs chez des individus atteints de MA au cours de la réalisation des tâches d'expérimentation. En premier lieu, la consigne fournie aux participants pour cette tâche est complexe et comprend des sous-consignes. Le participant est en effet invité à effectuer un café instantané avec lait et sucre ainsi qu'une rôtie avec beurre et confiture (voir Appendice C). La longueur de la consigne peut mener à une surcharge de la mémoire de travail et donc à des omissions dans la séquence. Les participants peuvent alors répéter certaines étapes de la tâche (persévérations). Ensuite, les participants n'ont pas accès à des indices visuels leur permettant de vérifier leurs actions ainsi que l'évolution de la tâche en cours afin de compenser pour les déficits d'ordre mnésique (ex. : ils ne peuvent vérifier s'ils ont déjà mis du sucre dans le café, car ce dernier s'est dissout; ou encore ne peuvent vérifier s'ils ont déjà mélangé le café ou non) (Wherton \& Monk, 2010). La troisième hypothèse est en lien avec l'émission de comportements non productifs (notamment la manipulation d'objets) augmentant le nombre d'étapes réalisées et pouvant s'expliquer par un problème d'ordre exécutif, une possible confusion ou encore de l'anxiété chez le participant. Finalement, il est à noter que la tâche café et rôtie peut être exécutée de deux façons différentes par les participants, soit séparément (exécution du café en premier lieu puis de la rôtie, ou vice versa) ou simultanément (exécution du café et de la rôtie de façon conjointe). L'expérimentation auprès du groupe contrôle démontre que 13 des 20 participants de l'échantillon ( $65 \%$ ) exécutent les deux tâches de façon simultanée. En ce qui concerne les participants atteints de MA, pour les 9 essais de réalisation d'un café et d'une rôtie (3 essais pour chacun des participants), 6 essais ont été réalisés 
simultanément $(66 \%)$. Certains participants ont utilisé les deux méthodes de résolution de la tâche (séparée ou simultanée) au cours de l'expérimentation et l'analyse descriptive des données indique que le nombre d'étapes réalisé est plus élevé lorsque le participant initie les deux tâches de façon simultanée. Cette tendance n'est pas observée auprès de l'échantillon contrôle.

En ce qui concerne les temps de réalisation, les analyses statistiques démontrent qu'il existe une différence significative entre le groupe contrôle et le groupe MA pour les temps 2 et 3 , alors que la différence pour le temps 1 ne s'avère pas significative. Laprise et al. (2010) ont noté une relation modérée $(r=0,425)$ entre l'âge des participants et le temps d'exécution chez un groupe contrôle. Les résultats obtenus dans le cadre de la présente étude en ce qui concerne le temps d'exécution de la tâche pourraient donc être influencés d'une part par l'âge du participant et, d'autre part, par les atteintes cognitives engendrées par la MA (vitesse de traitement de l'information, fonctionnement mnésique et exécutif, etc.). L'émission de comportements répétitifs ou persévératifs, les erreurs commises ainsi que l'introduction de guidages peuvent également avoir une influence sur les temps de réalisation de la tâche. Aucune autre étude connue à ce jour n'a cependant évalué l'influence de telles variables sur les temps de réalisation de la tâche.

En somme, les analyses statistiques révèlent que les résultats de la comparaison de performance de participants MA et d'un groupe contrôle peuvent varier dans le temps en ce qui concerne les variables étudiées, dans ce cas le nombre d'étapes ainsi que le temps 
de réalisation. En effet, aucune différence n'est mesurée entre les deux groupes au temps 1, mais des écarts significatifs sont mesurés aux temps 2 et 3 . De futures études pourraient donc vérifier si ces résultats sont également observables chez un plus grand nombre de participants MA et, si tel est le cas, tenter d'expliquer de tels phénomènes de même que les facteurs impliqués.

\section{Expérimentation : tâche cadeau}

Le nombre moyen d'étapes réalisées par les participants MA se rapproche davantage de la performance de l'échantillon contrôle pour la tâche d'emballage d'un cadeau que pour celle du café. Une différence significative est mesurée entre les deux groupes pour ce qui est du temps 1 , alors que les résultats aux temps 2 et 3 n'indiquent pas de différence significative. Une des hypothèses explicatives à considérer afin d'expliquer le faible écart entre le nombre d'étapes de l'échantillon contrôle et celui de l'échantillon clinique aux temps 2 et 3 est le fait que la consigne soumise aux participants est une consigne simple (en comparaison à la consigne de réalisation d'un café et d'une rôtie qui est une consigne complexe). Les possibilités pour le participant d'oublier une partie de la consigne sont donc diminuées, car la mémoire de travail se retrouve moins surchargée. De plus, la réalisation de la tâche d'emballage d'un cadeau ne comprend pas la possibilité d'interchanger certaines étapes de la séquence (dans la réalisation du café, le sucre peut être ajouté avant le lait et vice versa), ce qui peut diminuer les répétitions d'étapes ainsi que les persévérations de la part des participants. Le fait que la séquence de réalisation d'emballage d'un cadeau soit linéaire et que les étapes soient peu 
interchangeables aide donc possiblement les participants lors de l'exécution, en plus du fait que ces derniers ont accès à des indices visuels leur permettant de se valider quant à l'évolution de la séquence (ex. le participant voit que le papier d'emballage est collé d'un côté de la boîte alors qu'il ne l'est pas de l'autre) (Wherton \& Monk, 2010). Le protocole proposé dans le NAT (Schwartz et al., 2002) concernant la tâche d'emballage d'un cadeau comprend cependant la présence de distracteurs sur la table de travail, élément pouvant complexifier la réalisation de la tâche et augmenter le nombre d'erreurs commises, surtout pour une population atteinte de déficits cognitifs (Giovannetti et al., 2002). Il n'est cependant pas observé dans le cadre de ce protocole expérimental que les participants avec MA commettent plus d'erreurs dans la tâche avec distracteurs (emballage d'un cadeau) que dans la tâche sans distracteur (café et rôtie).

En ce qui concerne le temps de réalisation de la tâche, il est observé que le temps moyen de réalisation d'emballage d'un cadeau pour les participants atteints de MA se rapproche davantage du temps moyen de réalisation des normaux que pour la tâche café et rôtie. Une hypothèse à considérer afin d'expliquer le faible écart est que les participants (groupe contrôle et clinique) ont la possibilité de réaliser cette tâche en accordant une importance à l'esthétisme (ce qui s'applique moins dans la tâche café et rôtie). Certains participants étaient en effet soucieux du choix de la couleur du chou en fonction du papier d'emballage ou encore de l'emplacement de celui-ci sur le cadeau. Ces comportements, qui ont davantage été observés chez les normaux, et plus particulièrement chez les femmes (Laprise et al., 2010), peuvent augmenter le temps de 
réalisation de la tâche, réduisant ainsi l'écart entre les temps de réalisation des deux groupes. Deux autres facteurs peuvent également expliquer pourquoi les temps moyens d'emballage d'un cadeau pour le groupe contrôle et le groupe MA ont des valeurs rapprochées. Ces facteurs sont d'une part le nombre d'étapes, qui influence les temps de réalisation, et d'autre part la nature de la tâche et de la consigne qui surcharge moins la mémoire de travail. Le temps d'exécution de la tâche d'emballage d'un cadeau demeure toutefois légèrement plus élevé chez les participants MA que chez le groupe contrôle, la différence n'est pas significative pour les temps 2 et 3, alors qu'elle l'est au temps 1 . Les atteintes cognitives combinées aux erreurs ainsi qu'à l'introduction de guidages pourraient contribuer à augmenter légèrement le temps d'exécution. Il en est de même pour l'âge des participants (relation modérée entre l'âge des participants et le temps d'exécution) (Laprise et al., 2010).

Tel qu'observé avec la tâche café et rôtie, les analyses statistiques de comparaison de groupe réalisées avec la tâche d'emballage d'un cadeau semblent indiquer que les résultats des variables «nombre d'étapes » et «temps de réalisation » varient au cours des essais. En effet, pour ces deux variables, des écarts significatifs sont mesurés au temps 1 , mais non aux temps 2 et 3 . À nouveau, il serait pertinent de reproduire l'étude auprès d'un plus grand nombre de participants afin de déterminer si ces variations sont dues à la taille de l'échantillon et vérifier les facteurs impliqués dans de telles variations. 


\section{Effet d'apprentissage}

Bien que les capacités d'apprentissages de nouvelles informations soient restreintes chez les individus atteints de MA (Giffard et al., 2008), l'analyse descriptive des résultats révèle de possibles effets d'apprentissage chez P2 et P3 (diminution du nombre d'erreurs) et chez P1 (diminution du nombre d'étapes). Les études ne sont pas unanimes en ce qui concerne l'effet d'apprentissage lors de la répétition d'une même tâche avec introduction de guidage. En effet, dans des études réalisées avec des participants atteints de démence, Bewernitz, Mann, Dasler et Belchior (2009) ainsi que Labelle et Mihailidis (2006) observent un effet d'apprentissage chez leurs participants, alors que Mihailidis et al. (2004) n'en observent pas. Ces études ont cependant été réalisées auprès d'échantillons atteints de différents types de démence à différents stades d'évolution. Les résultats de la présente étude démontrent également qu'à plusieurs reprises, le nombre d'étapes effectuées ou encore le nombre d'erreurs commises par les participants a augmenté au cours des essais. Giffard et al. (2008) reconnaissent que la MA entraîne des troubles de nature exécutive et mnésique ainsi qu'une désorientation temporelle, spatiale et personnelle favorisant la confusion lors de tâches complexes, ce qui pourrait expliquer une dégradation des performances, soit une augmentation du nombre d'étapes et du nombre d'erreurs et ce, même lors de la répétition d'une même tâche.

Les prochaines sections ont pour objectif d'explorer les facteurs ayant influencé les taux de réponse aux différents types de guidages. Plusieurs pistes d'explication seront soumises afin d'améliorer la compréhension du phénomène et des paramètres de 
maximisation de l'efficacité des guidages applicables à de futures études seront proposés dans la section Forces et faiblesses de l'étude.

Question de recherche \# 1 : Parmi les trois méthodes de guidage à l'étude, laquelle

s'avère plus efficace chez les participants avec MA en stade modéré pour deux AVQ (préparation d'un café et d'une rôtie et emballage d'un cadeau)?

\section{Guidage visuel}

Le guidage visuel s'est avéré inefficace pour l'ensemble des participants et plusieurs hypothèses peuvent être considérées afin d'expliquer ces résultats. Rappelons d'abord que ce type de guidage est constitué d'un son indicateur précédant une vidéo d'un individu exécutant une séquence d'actions, ce son visant à attirer l'attention du participant sur l'écran. Au cours de la présentation de la tâche, les participants n'ont pas été avisés qu'un écran positionné devant eux pourrait émettre des sons et afficher des vidéos au cours de la réalisation de la tâche. De ce fait, lorsque le guidage était introduit, soit les participants ignoraient le son, soit ils éprouvaient de la difficulté à en localiser la provenance et le guidage s'avérait parfois déjà terminé lorsque le participant regardait l'écran. Par conséquent, les participants n'ont pu bénéficier du guidage visuel de façon optimale et c'est pourquoi il s'avère difficile, dans les conditions actuelles, de statuer sur son efficacité réelle. Labelle et Mihailidis (2006) de même que Bewernitz et al., (2009) ont également observé au cours de leur étude que les participants ne regardaient pas l'écran de façon systématique lors de la présentation de guidages audiovisuels. Toutefois, 
les participants étaient en mesure d'accomplir la tâche en utilisant seulement la partie verbale du guidage audiovisuel.

\section{Guidage verbal et audiovisuel}

Rappelons que les guidages qui se sont avérés partiellement efficaces pour les participants sont les guidages verbal et audiovisuel. À l'heure actuelle, les études réalisées à l'aide de la technologie $\mathrm{COACH}$ (Mihailidis et al. 2008) démontrent que le taux de réponse au guidage varie entre les participants, ce qui est également le cas dans la présente expérimentation. Dans une étude réalisée auprès de participants atteints de différents types de démence en stade modéré, Mihailidis et al. (2008) énoncent notamment que le nombre d'étapes complétées de façon indépendante lors des phases d'intervention avec guidage varie entre 0 et $26 \%$. Les facteurs explicatifs des variations d'efficacité des guidages demeurent encore à l'étude. De plus, Labelle et Mihailidis (2006) observent que certains participants bénéficient davantage du guidage audiovisuel (en comparaison avec le guidage verbal) ce qui est notamment le cas de P1. Les résultats de Labelle et Mihailidis (2006) ne s'avèrent cependant pas significatifs pour l'ensemble de l'échantillon.

Dans le même ordre d'idée, les guidages n'ont pas eu d'effet préjudiciable chez les participants, ni dans les études utilisant la technologie $\mathrm{COACH}$, ni au cours de la présente expérimentation. Ces derniers n’ont pas suscité de réaction négative (ex.: panique, agitation, impression d'hallucination auditive), ils représentent par conséquent 
une solution intéressante afin de soutenir efficacement la réalisation d'AVQ auprès de patients atteints de MA. De plus, il s'avère important de souligner que pour deux des trois participants, soit P1 et P3, les taux d'efficacité des guidages audiovisuels sont plus élevés que les taux d'inefficacité $(100$ - taux de guidage efficace $=$ taux de guidage inefficace), élément laissant supposer que ce type de guidage pourrait également être efficace chez d'autres patients atteints de MA. De futures études pourront corroborer cet aspect.

En ce qui concerne les guidages verbaux, deux hypothèses sont à considérer afin d'expliquer leur efficacité partielle. En premier lieu, l'attention du participant n'était pas mobilisée lors de l'introduction du guidage. Fréquemment, le participant ne constatait pas que le guidage s'adressait à lui et l'ignorait. Dans les cas où un participant comprenait que le guidage s'adressait à lui, une partie du message transmis par l'ordinateur pouvait ne pas avoir été entendue ou comprise. À une reprise, un participant a d'ailleurs demandé de répéter le guidage en raison du fait qu'il n'avait pas saisi l'entièreté du message. De même, l'environnement d'expérimentation comprenait, en plus du matériel, deux assistants de recherche ainsi que le membre de la famille accompagnateur (afin de sécuriser le participant). Il est alors possible que le participant ait cru que le guidage provenait d'une personne dans l'environnement d'expérimentation etlou s'adressait à l'un d'eux. Mihailidis et al. (2004) énoncent également que certains participants de leur étude n'ont pas constaté qu'ils recevaient une assistance informatique, ils ont plutôt perçu qu'ils étaient aidés par quelqu'un se situant dans la 
même pièce. La seconde hypothèse concerne l'aspect de la nouveauté. En effet, deux des participants ont manifesté à une reprise une réaction de surprise suite au guidage verbal. Ces derniers se questionnaient à savoir d'où provenait la voix entendue. À nouveau, ne sachant pas que le guidage leur était destiné, les participants ne l'exécutaient pas de façon constante.

Certaines hypothèses sont également à considérer afin d'expliquer l'efficacité partielle des guidages audiovisuels. En plus des hypothèses mentionnées précédemment concernant le guidage verbal, il est important de rappeler que, tout comme avec le guidage visuel, les participants n'étaient pas informés de la possibilité d'interventions informatiques sous forme de guidage au courant de la réalisation de leur tâche. De ce fait, en modalité audiovisuelle, même si les participants entendaient la partie verbale du guidage, ils n'étaient pas sensibilisés quant au fait de lever les yeux vers l'écran afin de bénéficier en plus du guidage vidéo. Donc, il n'est pas assuré que le participant ait profité de la combinaison des deux modalités (visuelle et verbale) du guidage audiovisuel. Ce qui peut cependant expliquer que les participants aient répondu aux guidages ayant une composante verbale (soit les guidages verbal et audiovisuel) est que ce guidage est accessible et qu'il ne nécessite pas un désengagement attentionnel de la tâche. Le participant peut donc poursuivre la réalisation de la séquence en cours tout en écoutant la consigne émise. O’Neil, Moran et Gillepsie (2009) énoncent d'ailleurs que le guidage visuel s'avère très exigeant sur le plan de l'attention visuelle. De ce fait, lorsqu'un participant est engagé dans une tâche de nature visuelle (ex.: habillage, 
préparation de repas), la présentation d'un guidage faisant appel à la même modalité nécessite une interruption de la tâche en cours ainsi qu'un désengagement attentionnel, ce qui augmente la charge cognitive. Le guidage visuel peut donc s'avérer une méthode cognitivement coûteuse pour laquelle l'efficacité pourrait être augmentée dans des conditions où le système visuel du participant est peu sollicité et que ce dernier est familier avec cette méthode d'intervention.

Dans tous les cas, les interventions de guidages peuvent représenter une source d'interférence dans la séquence d'action et puisque les capacités attentionnelles sont altérées dans la MA, il s'avère important de s'assurer que les interventions de guidages soient assimilables et nécessitent un investissement attentionnel minimal de la part du participant. L'évaluation neuropsychologique s'avère en ce sens une avenue de choix dans l'implantation de telles technologies, car elle permet l'évaluation des capacités spécifiques de chaque participant à un stade particulier de la maladie et ainsi la mise en place d'interventions de guidage appropriées à son profil (approche individualisée).

Question de recherche \#2: Est-il possible d'expliquer les principes relatifs d'efficacité du guidage par les atteintes neuropsychologiques des participants?

Cette section de l'étude se veut une discussion clinique effectuant des liens entre l'efficacité des guidages ainsi que le profil neuropsychologique des participants. L'objectif est de soumettre des hypothèses explicatives des résultats obtenus et de 
générer des pistes de recherche futures visant à améliorer la compréhension de la problématique.

Les résultats de l'étude démontrent des taux d'efficacité différents en regard du guidage pour tous les participants. P2 démontre les taux d'efficacité les plus bas, P1 démontre des taux d'efficacité intermédiaires alors que P3 démontre des taux d'efficacité optimaux. De plus, les profils neuropsychologiques des participants diffèrent également en termes d'atteintes et de capacités préservées. P2 est le participant présentant les déficits les plus importants, suivi de P3 et P1 démontre le moins d'atteintes neuropsychologiques.

En analysant les données de façon descriptive, il est possible d'établir, dans un premier temps, que deux participants démontrent des profils cognitifs relativement semblables, soit P1 et P3. En effet, ces deux participants se situent au même stade de la maladie (stade 4 selon la GDS) et la seule composante cognitive les distinguant est la présence chez P3 d'une apraxie idéomotrice. Les taux de réponse au guidage pour ces participants differrent cependant de façon importante, P1 obtient des taux de $33 \%$ (verbal) et $60 \%$ (audiovisuel), alors que P3 montre des taux de $100 \%$ pour les deux types de guidages. Les taux d'efficacité de P3 concernent toutefois uniquement la tâche café et rôtie (seulement une erreur avait été commise en tâche cadeau et le guidage visuel n'avait pas fonctionné), alors que les taux mentionnés pour P1 concernent les deux tâches. Différentes pistes d'explications des performances ont été avancées dans la 
section résultats, notamment, pour $\mathrm{P} 1$, l'addition de la composante visuelle à la composante verbale qui pourrait favoriser le taux de réponse au guidage audiovisuel en raison des capacités visuelles préservées. Pour P3, le guidage audiovisuel devrait également être favorisé pour les mêmes raisons. Cependant, le peu d'erreurs commises aurait pu influencer les taux de réponse au guidage.

Dans un deuxième temps, il est possible d'effectuer un lien entre les déficits neuropsychologiques de $\mathrm{P} 2$, le stade d'évolution de la maladie ainsi que les taux d'efficacité du guidage. En effet, ce participant démontre le plus d'atteintes cognitives, se situe à un stade d'évolution plus avancé que les autres participants et possède les taux d'efficacité les plus bas ( $14 \%$ en modalité audiovisuelle et $20 \%$ en modalité verbale). Il est également le seul participant pour qui le guidage verbal a été le plus efficace. L'atteinte de la sphère visuelle pourrait rendre difficile pour ce participant l'interprétation de cette portion du guidage, ce qui expliquerait en partie la plus grande efficacité du guidage verbal comparativement au guidage audiovisuel.

Dans le cadre de cette étude, les profils neuropsychologiques des participants ont été utilisés afin d'explorer les principes relatifs d'efficacité du guidage. L'exploration des données est peu révélatrice, notamment en raison du petit échantillon expérimental, mais d'autres facteurs contributifs ont également été identifiés. En effet, l'évaluation neuropsychologique à visée diagnostique (dans ce cas pour la MA), et ayant été utilisée dans cette étude, ne serait pas suffisante à l'explication du phénomène d'efficacité du 
guidage. En effet, elle ne mesurerait pas l'ensemble des prérequis nécessaires à la réception, la compréhension et l'exécution du guidage chez les participants. En faisant référence à un modèle de traitement de l'information (Wickens, 1992) (voir Appendice G) et en le transposant à des interventions de guidage, les différentes phases impliquées lors d'une intervention de guidage peuvent être identifiées, soit la phase perceptuelle, la phase cognitive et la phase exécutive (exécution comportementale de la réponse). $\mathrm{Ce}$ modèle permet de reconnaître l'ensemble des étapes nécessaires au traitement du guidage par les participants, de même que les prérequis cognitifs qui y sont rattachés. L'évaluation neuropsychologique ayant comme objectif l'instauration de technologies compensatoires devrait donc utiliser des outils d'évaluation visant à déterminer l'existence de déficits au sein des fonctions cognitives identifiées comme prérequis aux guidages.

Voici les composantes cognitives impliquées en fonction des trois modalités de guidage, extrapolées selon le modèle de traitement de l'information de Wickens (1992). Dans la phase perceptuelle, la perception du guidage visuel requiert la vision ainsi que le bon fonctionnement des capacités perceptuelles visuelles. Dans le cas du guidage verbal, l'audition ainsi que la compréhension verbale sont impliquées. Le guidage audiovisuel, quant à lui, fait appel aux fonctions nécessaires à la réception du guidage visuel en plus du guidage verbal. La phase cognitive requiert le fonctionnement de la mémoire de travail ainsi que des différentes formes de mémoire, de même que des fonctions exécutives dans la prise de décision de la réponse adéquate à émettre (dans tous les types 
de guidage). Dans la phase exécutive (exécution comportementale de la réponse), les capacités de recherche visuelle (incluant l'attention sélective), la reconnaissance d'objets (gnosie) ainsi que de manipulation (praxies idéatoires et idéomotrices) doivent être préservées peu importe la modalité de guidage. Les capacités attentionnelles chapeautent évidemment les trois phases tout au long du processus. En somme, l'évaluation neuropsychologique visant à évaluer l'efficacité de différents types de guidage devrait inclure l'ensemble des composantes ci-haut mentionnées et ce, afin d'obtenir les informations pertinentes quant aux prérequis cognitifs nécessaires à la réception et la mise en cuvre du guidage. Ainsi, lors de l'implantation du guidage dans l'environnement des patients, les interventions de guidages pourraient être personnalisées en fonction du profil neuropsychologique du patient, ce qui en augmenterait l'efficacité.

L'utilisation du modèle de traitement de l'information en tant qu'outil de compréhension des guidages permet d'explorer certaines hypothèses en lien avec l'efficacité du guidage et le profil neuropsychologique. En effet, afin d'être efficace, le guidage, peu importe la nature, devrait faire appel à certaines capacités dites résiduelles (capacités non atteintes par des déficits, c'est-à-dire toujours fonctionnelles). Dans le cas de P1 et P3, le taux d'efficacité des guidages audiovisuels est plus important que le taux d'inefficacité. Il peut donc être postulé que ces participants possèdent certaines des capacités résiduelles afin de prendre en charge les différentes composantes liées à la réception et à l'exécution de guidages. Cependant, dans le cas de P2, le taux 
d'inefficacité du guidage est plus élevé que le taux d'efficacité. Il est donc probable que ce participant n'ait pas 1'ensemble des capacités résiduelles nécessaires à la réception et à l'exécution du guidage, par exemple en raison de l'évolution de la maladie. Les résultats obtenus pour ce participant questionnent donc l'existence possible d'une période d'efficacité maximale au-delà de laquelle les guidages testés dans cette étude pourraient s'avérer inefficaces. Si tel était le cas, d'autres types de guidages ou pistes d'interventions devraient être explorés en ce qui concerne ce participant.

En plus des prérequis cognitifs au guidage, d'autres pistes s'avèrent intéressantes à considérer afin d'expliquer l'efficacité de certains types de guidage par rapport à d'autres. Par exemple, Nailos et al. (1994) abordent la problématique d'efficacité des guidages pour des individus avec retard mental (guidages effectués par des assistants et non de façon informatisée) en terme de profil du participant et de caractéristique de la tâche. Selon Van Tassel et al. (2011), les sous-composantes à considérer en ce qui concerne le profil du participant seraient le diagnostic, les habiletés personnelles prémorbides ainsi que les déficits cognitifs spécifiques, alors que les caractéristiques de la tâche pourraient être abordées en terme de nature, de familiarité et de complexité. L'analyse des données de la présente étude tient compte de plusieurs de ces facteurs, mais omet notamment les habiletés personnelles prémorbides (ex : davantage verbale que visuelle ou vice versa). Wherton et Monk (2010) abordent quant à eux la présentation du guidage en fonction du type d'erreurs commises par les participants. Le type d'erreurs commises informerait notamment sur le genre d'intervention à émettre 
(préventif ou correctif) ainsi que l'approche à utiliser (implicite ou explicite). En somme,

l'évaluation de l'efficacité de guidages pourrait reposer sur différentes variables qu'il serait pertinent de considérer de façon concomitante.

\section{Forces et faiblesses de l'étude}

Une des forces de l'étude concerne les critères de sélection de l'échantillon clinique. En effet, comparativement aux autres études effectuées avec la technologie $\mathrm{COACH}$ (Miahailidis et al., 2008), la sélection de l'échantillon expérimental a été effectuée en fonction d'un type de démence, soit la maladie d'Alzheimer, ainsi qu'un stade précis, soit le stade modéré. Le stade de la maladie a été déterminé en fonction de la Global deterioration scale (GDS) et non avec le MMSE qui s'avère un indicateur peu fiable du stade de la démence (Derouesné, 2006; Lopez et al., 2005). La détermination de tels critères de sélection favorise l'homogénéité de l'échantillon ainsi que la validité des résultats.

L'utilisation d'un échantillon contrôle représente un avantage, car il permet la comparaison des données obtenues auprès de l'échantillon clinique et offre la possibilité de réaliser des analyses statistiques. Le devis expérimental, qui comprend deux tâches différentes (café et rôtie ainsi que l'emballage d'un cadeau), représente également une force comparativement aux autres études du même type qui en ont utilisé qu'une seule. En plus d'augmenter les données recueillies, le fait que les deux tâches soient différentes 
permet d'effectuer des liens en ce qui concerne la nature de la tâche à réaliser et les performances des participants.

Une autre des forces se situe au niveau de l'approche théorique utilisée dans la compréhension de l'efficacité du guidage, soit l'approche neuropsychologique. En effet, à notre connaissance, aucune autre étude effectuée préalablement n'a envisagé d'aborder la question de l'efficacité du guidage en fonction des atteintes neuropsychologiques révélées à l'aide d'une évaluation. Ce champ de recherche est donc novateur.

Certaines faiblesses méthodologiques sont à relever en ce qui concerne la présente expérimentation. En premier lieu, un des participants a été exclu de l'échantillon, car aucune erreur n'avait été commise lors de la réalisation des tâches. La réplication de cette étude devrait soit évaluer dans un premier temps le degré d'indépendance des participants dans une tâche sans introduction de guidage, soit sélectionner des tâches comprenant un niveau de difficulté plus élevé afin d'assurer que le participant commette des erreurs dans la tâche (qui permettront l'évaluation de l'efficacité du guidage). L'ajout de questions précises concernant la réalisation d'AVQ ainsi que des tâches utilisées dans la phase expérimentale au sein du questionnaire sociodémographique pourrait également favoriser le dépistage des candidats. Cela permettrait en plus d'effectuer des liens entre les capacités de réalisation d'AVQ à domicile, le taux d'erreur commise ainsi que le l'efficacité des guidages. 
Une autre faiblesse méthodologique identifiée concerne la formulation des interventions de guidage pour certains types d'erreurs commises par les participants. En effet, l'introduction de guidages lors d'erreurs de répétition d'étapes ou encore d'erreurs de type persévératif effectuées à des moments précis de la tâche (ex : lors de la dernière étape de la tâche d'emballage d'un cadeau ou lorsqu'une rôtie est déjà dans le grille-pain) ne s'est pas avéré aidante pour le participant. Les guidages indiquent l'étape suivante à effectuer, mais dans les cas où la tâche s'avère complète et que le participant persévère dans l'action (par exemple si le participant persévère à apposer des choux sur le cadeau), aucun guidage ne peut être utilisé. Dans le même ordre d'idée, lorsque la rôtie est dans le grille-pain, aucun guidage n'est prévu pour indiquer au participant qu'il doit attendre quelques minutes. Ainsi, un guidage indiquant que l'étape en cours a été complétée avec succès et que cela complète la tâche ou que l'étape en cours a été complétée avec succès et que le participant doit attendre avant de passer à la prochaine étape devrait être créé afin de maximiser l'efficacité des interventions.

Toujours sur le plan méthodologique, un troisième élément à améliorer concerne les images servant d'aide mémoire lors de l'exécution des tâches comprises dans le protocole du NAT. En effet, ces images représentent le résultat final de la tâche sous forme d'un dessin en noir et blanc, soit un café et une rôtie beurrée ainsi qu'un cadeau emballé. L'expérimentation a démontré que le dessin représentant le cadeau emballé pouvait créer de la confusion chez les participants puisqu'il n'était pas identique au résultat attendu (au niveau du graphisme du papier d'emballage ainsi que de la forme 
des choux à apposer sur le cadeau). C'est pourquoi il est suggéré que ce dessin soit adapté au matériel disponible lors de l'expérimentation.

Deux incidents ayant des répercussions sur les données expérimentales se sont produits au cours du temps deux (pour les participants 2 et 3) et ce, malgré que des précautions aient été mises en place (vérification du matériel informatique préalable à l'expérimentation, formation rigoureuse des assistants de recherche). Pour le participant 2, le matériel d'expérimentation a été disposé incorrectement sur la surface de travail à deux reprises faisant en sorte que trois étapes de moins étaient nécessaires à la réalisation de la tâche d'emballage d'un cadeau. Pour le participant 5, une problématique informatique est survenue et l'introduction du guidage visuel a été impossible lors de la réalisation d'un café et d'une rôtie. Lors de ces incidents, la décision de ne pas reprendre les tâches en cours a été prise en raison du fait que les participants auraient bénéficié d'un plus grand nombre d'essais, ce qui aurait pu avoir des répercussions sur la familiarisation avec les différentes tâches ainsi que le nombre d'erreurs commises.

Les présentations de guidages au cours de la phase expérimentale se sont avérées inefficaces dans plusieurs des cas, notamment en raison du fait que le participant n'était pas préparé à recevoir de telles interventions et que les processus attentionnels n'étaient pas suffisamment mobilisés avant l'introduction du guidage. Certains éléments seraient par conséquent à améliorer et ce, afin d'assurer que le participant bénéficie des guidages selon leur plein potentiel. En premier lieu, les participants devraient être sensibilisés à la 
présence de matériel informatique et un court entraînement pourrait être effectué afin de démontrer le type d'interventions susceptibles d'être émises. Cela pourrait diminuer, voire éliminer les réactions de surprise chez les participants lors d'introduction de guidage. Ensuite, afin de maximiser le potentiel d'efficacité des guidages, le nom du participant pourrait être utilisé avant l'introduction de guidages afin de le mobiliser et ainsi attirer son attention. L'ajout du rappel de la tâche en cours ainsi que l'implantation d'indications plus détaillées sur l'action à effectuer pourraient s'avérer bénéfiques. Ces recommandations sont également mentionnées dans les études utilisant la technologie COACH (Mihailidis et al., 2008).

Finalement, le petit échantillon expérimental peut être considéré comme une faiblesse. En effet, un échantillon plus représentatif de la population aurait permis d'effectuer des analyses statistiques pour l'ensemble des données et ainsi favoriser la généralisation des résultats. De nouvelles avenues sont d'ailleurs explorées afin d'améliorer les méthodes de recrutement de participants qui serviront à poursuivre la recherche dans ce domaine au sein du LIARA (Laboratoire d'Intelligence Ambiante pour la Reconnaissance d'Activité) de l'UQAC.

\section{Analyse des conséquences de la recherche et retombées possibles}

Cette étude de cas multiples avait comme but d'explorer une nouvelle compréhension du guidage informatisé à l'aide d'un point de vue neuropsychologique et un premier pas a été réalisé dans cette direction auprès de trois participants atteints d'Alzheimer en 
stade modéré. En utilisant cette compréhension et en intégrant les suggestions d'amélioration de la recherche émises dans la présente section, d'autres études pourront se pencher sur le même type de problématique en recrutant un plus grand échantillon clinique de façon à généraliser les résultats. Lorsque 1'efficacité de certains types de guidage aura été démontrée de façon empirique, il sera possible d'instaurer les technologies d'assistance au sein même des environnements des patients atteints de MA, soit à domicile ou encore en milieu hospitalier. L'instauration de guidages informatisés, combinés avec l'utilisation de traitements pharmacologiques visant la moduler le déclin cognitif lié à la MA, auraient le potentiel de compenser temporairement les altérations cognitives des patients atteints. De cette façon, la charge incombant aux aidants ou encore au personnel de soin serait diminuée, les systèmes d'intelligence ambiante pouvant soutenir efficacement le patient dans différentes AVQ. Il est admis que les technologies d'assistance ne pourront éventuellement pas remplacer la présence d'un aidant, mais l'institutionnalisation des patients demeurant à domicile pourrait être retardée et les coûts reliés au système de santé réduits. 
En conclusion, cette étude de cas multiples avait comme objectif d'évaluer l'efficacité de trois méthodes de guidages informatisés (visuel, verbal et audiovisuel) ainsi que d'explorer la contribution du profil neuropsychologique dans l'explication de l'efficacité des guidages. Le protocole expérimental, qui a été testé auprès de trois participants atteints de maladie d'Alzheimer en stade modéré, s'avère novateur en raison de la comparaison des performances avec un groupe contrôle ainsi que de l'utilisation de deux AVQ différentes. Le petit échantillon clinique permet une analyse détaillée des performances des participants aux différentes tâches ainsi que des phénomènes impliqués dans l'efficacité des guidages. Cette étude le également le potentiel d'être répliquée auprès d'un échantillon plus représentatif d'une population atteinte de MA et ce, afin de rendre les résultats généralisables. Finalement, malgré le fait que peu de conclusions peuvent être tirées de cette étude concernant les liens entre le profil neuropsychologique mesuré et l'efficacité des guidages, l'apport d'une compréhension théorique à l'aide d'un modèle de traitement de l'information de même que les recommandations émises dans la discussion seront largement utiles dans le développement et la mise en œuvre de d'autres projets de recherche dans des domaines d'activité similaires. 
Références 
Adunsky, A., Fleissig, V., Levenkrohn, M., Arad, M., \& Noy, S. (2002). A comparative study of Mini-Mental Test, Clock Drawing Task and Cognitive-FIM in evaluating functional outcome of elderly hip fracture patients. Clinical Rehabilitation, 16, 414419.

Agniel, A., Joanette, Y., Doyon, B., \& Duchein, C. (1992). Protocole MontréalToulouse d'évaluation des gnosies visuelles. France: L'Ortho-Édition.

Allain, P., \& Le Gall, D. (2008). Approche théorique des fonctions exécutives. Dans O. Godefroy et les membres du GREFEX (Éds.), Fonctions exécutives et pathologie neurologiques et psychiatriques (pp.9-42). Marseille : Solal.

Anderson, R. (1994). Practionner's guide to clinical neuropsychology. New York: Plenium Press.

Antoine, C., Antoine, C., Guermonprez, P., \& Frigard, B. (2004). Awareness of deficits and anosognosia in Alzheimer's disease. Encephale, 30(6), 570-577.

American Psychological Association. (2004). Diagnostic and statistical manual of mental disorders (4e éd. texte révisé). Virginie: APA.

Axelrod, B. N., Ricker, J. H., \& Cherry, S. A. (1994). Concurrent validity of the MAE visual naming test. Archives of Clinical Neuropsychology, 9, 317-321.

Bayard, S., Derouesné, C., \& Gély-Nargeot, M.-C. (2006). Apraxies et démences. Dans C. Belin, A.-M. Ergis \& O. Moreaud (Éds), Actualités sur les démences: Aspects cliniques et neuropsychologiques (pp.199-207). Marseille : Solal.

Becker, J. T. (1988). Working memory and secondary memory deficits in Alzheimer's disease. Journal of clinical and experimental neuropsychology, 10, 739-753.

Bewernitz, M. W., Mann, W. C., Dasler, P., \& Belchior, P. (2009). Feasability of machine-based prompting to assist persons with dementia. Assistive Technology, 21(4), 196-207.

Bherer, L., Belleville, S., \& Hudon, C. (2004). Le déclin des fonctions exécutives au cours du vieillissement normal, dans la maladie d'Alzheimer et dans la démence frontotemporale. Psychologie \& NeuroPsychiatrie du Vieillissement, 2(3), 181-189. 
Boger, J., Hoey, J., Poupart, P., Boutilier, C., Fernie, G., \& Mihailidis, A. (2006). A planning system based on markov decision processes to guide people with dementia trough activities of daily living. IEEE Transaction on Information Technology in Biomedicine, 10(2), 323-333.

Botez-Marquad, T., \& Boller, F. (2005). Neuropsychologie clinique et neurologie du comportement (3e éd.). Montréal : Les Presses de l’Université de Montréal.

Bouchard, B., Bouzouane, A., \& Giroux, S. (2007). A keyhole plan recognition model for Alzheimer's patients: First results. Journal of Applied Artificial Intelligence, 22(7), 623-658.

Buschke, H. (1984). Cued recall in amnesia. Journal of Clinical Neuropsychology, 6, 433-440.

Cardebat, D., Aithamon, B., \& Puel, M. (1995). Les troubles du langage dans les démences de type Alzheimer. Dans F. Eustache et A. Agniel (Éds), Neuropsychologie clinique des démences : Évaluations et prises en charge (pp. 213-223). Marseille: Solal.

Chen, S. T., Sultzer, D. L., Hinkin, C. H., Mahler, M. E., \& Cummings, J. L. (1998). Executive dysfunction in Alzheimer's disease: Association with neuropsychiatric symptoms and functional impairment. The Journal of Neuropsychiatry anc Clinical Neurosciences, 10, 426-432.

Collectif Larousse (2007). Encyclopédie médicale - Le manuel Merck. Paris : Les Éditions Larousse.

Crum, R. M., Anthony, J. C., Bassett, S. S., Folstein, M. F. (1993). Population-based norms for the Mini-Mental State Evaluation by age and education level. Journal of the American Medecine Association, 269(18), 2386-2391.

Cummings, J. L. (1990). Clinical diagnosis of Alzheimer's disease. Dans J. L. Cummings \& B. L. Milner (Éds), Alzheimer's disease: Treatment and long-term management (pp.3-22). New York: Marcel Dekker.

Cutchin, M. (2003). The process of mediated aging-in-place: A theoretically and empirically based model. Social Science and Medicine, 57, 1077-1090.

Dartigues, J.-F., Berr, C., Helmer, C., \& Letenneur, L. (2002). Épidémiologie de la maladie d'Alzheimer. Médecine/Sciences, 18, 737-743.

DeKostsky, S. T., \& Orgogozo, J. M. (2001). Alzheimer disease: Diagnosis, costs, and dimensions of treatment. Alzheimer Disease and Associated Disorders, 15, S3-7. 
Della Sala, S., Lucchelli, F., \& Spinnler, H. (1987). Ideomotor apraxia in patients with dementia of the Alzheimer type. Journal of Neurology, 234, 91-93.

Derouesné, C. (2006). Maladie d'Alzheimer: Données épidémiologiques, neuropathologiques et cliniques. Dans C. Belin, A.-M. Ergis \& O. Moreaud (Éds), Actualités sur les démences: Aspects cliniques et neuropsychologiques (pp.25-34). Marseille : Solal.

Diamond, J. (2006). Rapport sur la maladie d'Alzheimer et la recherche actuelle, Société Alzheimer du Canada, 1-23.

Engelman, K. K., Altus, D. E., Mosier, M. C., \& Mathews, R. M. (2003). Brief training to promote the use of less intrusive prompts by nursing assistant in a dementia care unit. Journal of Applied Behavior Analysis, 36(1), 129-132.

Espino, D. V., Lichtenstein, M. J., Palmer, R. F., \& Hazuda, H. P. (2004). Evaluation of the Mini-Mental State Evaluation's internal consistency in a community-based sample of Mexican-American and European-American elders: Results from San Antonio longitudinal study of aging. Journal of the American Geriatric Society, 52, 822-827.

Eustache, F., \& Desgranges, B. (1995). La mémoire à long terme dans la maladie d'Alzheimer. Dans O. Eustache \& A. Agniel (Éds), Neuropsychologie clinique des démences : Évaluations et prises en charge (pp.183-197). Marseille : Solal.

Fals-Stewart, W. (1991). An interrater reliability study of the Trail Making Test (Part A and $B$ ). Unpublished manuscript.

Fastenau, P. S., Denburg, N. L., \& Mauer, B. A. (1998). Parallel short forms of the Boston Naming Test: Psychometric properties and norms for older adults. Journal of Clinical and Experimental Neuropsychology, 20(6), 828-834.

Flanagan, J. L., \& Jackson, S. T. (1997). Test-retest reliability of three aphasia test: Performance of non-brain-damaged older adults. Journal of Communication Disorders, 30, 33-43.

Folstein, M. F., Folstein, S. E., McHugh, P. R. (1975). "Mini-mental state": A practical method for grading the cognitive state of patients for the clinician. Journal of Psychiatric Research, 12(3), 189-198.

Folstein, M. F., Folstein, S. E., McHugh, P. R., \& Fanjiang, G. (2001). Mini-Mental State Examination user's guide. Odessa: Psychological Assessment Resources. 
Fougeyrollas, P., Cloutier, R., Bergeron, H., Côté, J., St-Michel, G. (1998). Classification québécoise du processus de production du handicap. Lac St-Charles: RIPPH/SCCIDIH.

Giffard, B., Desgranges, B., \& Eustache, F. (2008). La maladie d'Alzheimer. Dans B. Lechevalier, F. Eustache \& F. Viader (Éds), Traité de neuropsychologie clinique (pp. 766-791). Bruxelle : De Boeck.

Giovannetti, T., Libon, D. J., Buxbaum, L. J., \& Schwartz, M. F., (2002). Naturalistic action impairment in dementia. Neuropsychologia. 40(8), 1220-1232.

Guéguen, B., Chauvel, P., \& Touchon, J. (2005). Neurosphysiologie des mémoires. Paris : Elsevier.

Heinik, J., Solomesh, I., \& Beckman, P. (2004). Correlation between the CAMCOG, the MMSE, and three clock drawing tests in a specialized outpatient psychogeriatric service. Archives of Gerontology and Geriatrics, 38, 77-84.

Hodges, J. R., \& Patterson, K. (1997). Semantic memory disorders. Trends in Cognitive Sciences, 1, 68-72.

Hopp, G. A., Dixon, R. A., Backman, I., \& Grut, M. (1997). Stability of two measures of cognitive functioning in nondemented old-old adults. Journal of Clinical Psychology, $53,673-686$.

Hugonot-Diener, L. (2007). Guide pratique de la consultation en gériatrie ( $2^{\mathrm{e}}$ éd.). Paris : Masson.

Jefferson, A. L., Barakat, L. P., Giovannetti, T., Paul, R. H., \& Glosser, G. (2006). Object perception impairment predict instrumental activities of daily living dependance in Alzheimer's disease. Journal of Clinical and Experimental Neuropsychology, 28, 884-897.

Joanette, Y., Ska, B., Poissant, A., Belleville, S., Bellavance, A., Gauthier, S. et al. (1995). Évaluation neuropsychologique et profils cognitifs des démences de type Alzheimer: Dissociations transversales et longitudinales. Dans F. Eustache \& A. Agniel (Éds), Neuropsychologie clinique des démences: Évaluations et prises en charge (pp. 91-106). Marseille: Solal.

Johansson, B., \& Zarit, S. H. (1991). Dementia and cognitive impairment in the oldest old: A comparison of two rating methods. International Psychogeriatrics, 3, 29-38.

Kaplan, E. F., Goodglass, H., \& Weintraub, S. (1983). The Boston Naming Test. Philadelphie : Lea \& Febiger. 
Khosravi, M. (2003). La communication lors de la maladie d'Alzheimer et des démences séniles : Parler, comprendre, stimuler, distraire. Rueil-Malmaison, Cédex : Doin.

L'Association québécoise d'établissements de santé et de services sociaux (2007). Consultation publique sur les conditions de vie des aînés : Favoriser des liens pour assurer de meilleures conditions de vie aux aînés. Bibliothèque nationale du Québec.

Labelle, K.-L., \& Mihailidis, A. (2006). The use of automated prompting to facilitate handwashing in persons with dementia. American Journal of Occupational Therapy, $60(4), 442-450$

Lagha Pierucci, A. (1996). Étude des réalisations gestuelles dans la maladie d'Alzheimer. Thèse de doctorat inédite, Université de Paris 06.

Laprise, H., Bouchard, J., Bouchard, B., \& Bouzouane, A. (2010). Creating tools and trial data sets for smart home researchers: Experimenting activities of daily living with normal subjects to compare with Alzheimer's patients. Proceedings of the International Conference IADIS e-Health 2010 (pp. 143-150). Freiburg, Germany.

Lechevalier, B., \& Viader, F. (2008). Approche anatomo-clinique des aphasies. Dans B. Lechevalier, F. Eustache \& F. Viader (Éds), Traité de neuropsychologie clinique (pp. 473-485). Bruxelle : De Boeck.

Lechowski, L., Dieudonné, B., Tortrat, D., Teillet, L., Robert, P. H., Benoit, M., Forette, B., Vellas, B., \& PHRC-REAL.FR. (2003). Role of behavioral disturbance in the loss of autonomy for activities of daily living in Alzheimer's patients. International Journal of Geriatric Psychiatry, 18, 977-982.

Levinoff, E. J., Murtha, S., Li, K. Z. H., \& Chertkow, H. (2004). Selective attention impairments in Alzheimer's disease: Evidence for dissociable components. Neuropsychology, 18(3), 580-588.

Lezak, M. D., Howieson, D. B., \& Loring, D. W. (2004). Neuropsychological assessment (4th Ed.). New York: Oxford University Press.

Logie, R. H., Della Sala, S., Cocchini, G., \& Baddeley A. C. (2004). Is there a specific executive capacity for dual task coordination? Evidence from Alzheimer's disease. Neuropsychology, 18(3), 504-513.

Lopez, M. N., Charter, R. A., Mostafavi, B., Nibut, L. P., \& Smith, W. E. (2005). Psychometric properties of the Folstein Mini-Mental State Examination. Assessment, $12(2), 137-144$. 
Mahurin, R. K., DeBettignies, B. H., \& Pirozzolo, J. F. (1991). Structured assessment of independant living skills: Preleminary report of a performance mesure of functional abilities in dementia. Journal of Gerontology, 46, 58-66.

McDougall, C. J. (1990). A review of sreening instruments for assessing cognition and mental status in older adults. The Nurse Practioner, 15, 18-28.

Mendez, M. F., Ala, T., \& Underwood, K. L. (1992). Development of scoring criteria for the clock drawing task in Alzheimer's disease. Journal of the American Geriatrics Society, 40, 1095-1099.

Meulemans, T., \& Seron, X. (2000). Évaluation dans le cadre de l'expertise medicolégale. Dans X. Seron \& M. Van der Linden (Éds), Traité de neuropsychologie clinique, Tome I (pp. 387-404). Marseilles: Solal.

Mihailidis, A., Barbenel, J. C., \& Fernie, G. (2004). The efficacy of an intelligent cognitvie orthosis to facilitate handwashing by persons with moderate to severe dementia. Neuropsychological Rehabilitation, 14(1/2), 135-171.

Mihailidis, A., Boger, J., Canido, M., \& Hoey, J. (2007). The use of an intelligent prompting system for people with dementia. Interactions, July-August, 34-37.

Mihailidis, A., Boger, J., Craig, T., \& Hoey, J. (2008). The COACH promptimg system to assist older adults with dementia through handwashing: An efficacy study. $B M C$ Geriatrics, 8(28).

Mitrushina, M., \& Satz, P. (1995). Repeated testing of normal elderly with the Boston Naming Test. Aging Clinical and Experimental Research, 7, 123-127.

Nailos, M. A., Whitman, T. L., Maxwell, S. E. (1994). Verbal and visual instructions with mental retardation: The role of subject characteristics and task factors. Journal of Behavioral Education, 4(2), 201-216.

Nestor, P. J., Scheltens, P., \& Hodges, J. R. (2004). Advances in the early detection of Alzheimer's disease. Nature Medicine, 10 Suppl, 34-41.

O'Connell, M. E., \& Tuokko, H. (2002). 12-item Buschke Memory Test : Appropriate for use across levels of impairment. Applied Neuropsychology, 9(4), 226-233.

O’Neil, B., Moran, K., \& Gillepsie, A. (2009). Scaffolding rehabilitation behaviour using a voice-mediated assistive technology for cognition. Neuropsychological Rehabilitation, 20(4), 509-527. 
Organisation Mondiale de la Santé (2004). Stratégie mondiale pour l'alimentation, l'exercice physique et la santé. France.

Overall, J. E., Scott, J., Rhoades, H. M., \& Lesser, J. (1990). Empirical scaling of the stages of cognitive decline in senile dementia. Journal of Geriatric Psychiatry and Neurology, 3, 212-220.

Paolo, A. M., Axelrod, B. N., Troster, A. L., Blackwell, K. T., \& Koller, W. C. (1996). Uitlity of a Wisconsin card sorting test in persons with Alzheimer's disease and Parkinson's disease. Journal of Clinical Experimental Neuropsychology, 18, 56-115.

Paquette, C. (2009). Guide des meilleures pratiques en réadaptation cognitive. Québec : Presses de l’Université du Québec.

Paul, R. H., Cohen, R. A., Moser, D. J., Zawacki, T., Ott, B. R., Gordon, N., \& Stone, W. (2002). The Global Deterioration Scale: Relationship to neuropsychological performance and activities of daily living in patient with vascular dementia. Journal of Geriatric Psychiatry and Neurology, 15, 50-54.

Perry, J., Galloway, S., Bottorf, J. L., \& Nixon, S. (2005). Nurse-patient communication in dementia: Improving the odds. Journal of Gerontological Nursing, 31(4), 43-52.

Perry, R. J. \& Hodges, J. R. (2000). Fate of patients with questionnable Alzheimer's disease : Longitudinal profiles of individual subjects' decline. Dementia and Geriatric Cognitive Disorders, 11, 342-349.

Pigot, H., Mayers, A., \& Giroux, S. (2003). The intelligent habitat and everyday life activity support. Proceedings of the $5^{\text {th }}$ International Conference on Simulation in Biomedicine (pp. 507-516). Ljubljana, Slovenia.

Potkin, S. G., (2002). The ABC of Alzheimer's disease: ADL and improving day-to-day functionning of patients. International Psychogeriatrics, 14(1), 7-26.

Raymer, A. M., \& Ochipa, C. (1997). Conceptual Praxis. Dans L. J. G. Rothi, \& K. M. Heilman (Éds), Apraxia: The Neuropsychology of Action (pp.51-60). Hove: Psychology Press.

Reisberg, B., Ferris, S. H., de Leon, M. J., \& Crook, T. (1982). The Global Deterioration Scale for the assesment of primary degenerative dementia. American Journal of Psychiatry, 139, 1136-1139.

Reitan, R. M. (1955). The relation of the Trail Making Test to organic brain damage. Journal of consulting psychology, 19, 393-394. 
Ribot, T. (1882). Diseases of the memory: An essay in the positive psychology. New York : D. Appleton and Company.

Royall, D. R., Mahurin, R. K, True, J. E., Anderson, B., Brock, I. P., Freeburger, L., \& Miller, A. (1993). Executive impairment among the functionally dependent: Comparisons between schizophrenic and elderly subjects. American Journal of Psychiatry, 150, 1813-1819.

Royan, J., Tombaugh, T. N., Rees, L., \& Francis, M. (2004). The Adjusting-Paced Serial Addition Test (Adjusting-PSAT): Thresholds for speed of information processing as a function of stimulus modality and problem complexity. Archives of Clinical Neuropsychology, 19, 131-143.

Salmon, D. P., \& Hodges, J. R. (2001). Neuropsychological assessment of early onset dementia. Dans J. Hodges (Ed.), Early-onset dementia: A multi-disciplinary approach (pp.47-73). New York: Oxford University Press.

Schenk, F., Leuba, G., \& Büla, C. (2004). Du vieillissement cérébral à la maladie d'Alzheimer. Paris : De Boeck Université.

Schwartz, M. F., \& Buxbaum, L. J. (1997). Naturalistic action. Dans L. J. G. Rothi \& K. M. Heilman (Éds), Apraxia : The neuropsychology of action, (pp. 269-289). Hove : Psychology Press.

Schwartz, M. F., Segal, M., Veramonti, T., Ferraro, M., \& Buxbaum, L. J. (2002). The Naturalistic Action Test: A standardised assessment for everyday action impairment. Neuropsychological Rehabilitation, 12(4), 311-339.

Serna, A., Pigot, H., \& Rialle, V. (2007). Modeling the progression of Alzheimer's disease for cogintive assistance in smart homes. User Modeling and User-Adapted Interaction, 17(4), 415-438.

Société Alzheimer du Canada (2009). La maladie d'Alzheimer. Récupéré le 2 juin 2009 de http://www.alzheimer.ca/french/disease/intro.htm

Spreen, O., \& Strauss, E. (1998). A compendium of neuropsychological test: Administration, norms and commentary (2nd ed.). New York: Orford University Press.

Storms, G., Saerens, J., \& De Deyn, P. P. (2004). Normative data for the Boston Naming Test in native Dutch-speaking Belgian children and the relation with intelligence. Brain and Language, 91, 274-281. 
Strauss, E., Sherman, E. M. S., \& Spreen, O. (2006). A compendium of neuropsychological test: Administration, norms, and commentary (3rd ed.). New York: Oxford University Press.

Swanberg, M. M., Tractenberg, R. E., Mohs, R., Thal, L. J., \& Cummings, J. L. (2004). Executive dysfunction in Alzheimer disease. Archives of Neurology, 61(4), 556-560.

Tekin, S., Fairbanks, L. A., O'Connor, S., Rosenberg S., \& Cummings, J. L. (2001). Activities of daily living in Alzheimer's disease. American Journal of Geriatric Psychiatry, 9(1), 81-86.

Tombaugh, N. T., Rees, L., \& McIntyre, N. (1996). Normative data for the trail making test. Personal communication.

Touchon, J., \& Portet, F. (2002). La maladie d'Alzheimer. Paris : Masson.

Tuokko, H., \& Crockett, D. (1989). Cued recall and memory disorder in dementia. Journal of Clinical and Experimental Neuropsychology, 11, 278-294.

Van Tassel, M., Bouchard, J., Bouchard, B., \& Bouzouane, A. (2011). Guidelines for increasing prompt efficiency in smart homes according to the resident's profile and task characteristics. Proceedings of the $9^{\text {th }}$ International Conference on Smart Home and Health Telematics (pp. 112-120). Montréal, Canada: Springer.

Verstichel, P., \& Cambier, J. (2006). Les aphasies. Dans T. Botez-Marguard \& F. Boller (Éds), Neuropsychologie clinique et neurologie du comportement (3e éd.) (pp.471500). Montréal: Les presses de l'Université de Montréal.

Viader, F., Eustache, F., Lechevalier, B. (2000). Espace, geste, action. Paris: De Boeck Université.

Wagner, M. T., Spangenberg, K. B., Bachman, D. L., \& O'Connell, P. (1997). Unawareness of cognitive deficit in Alzheimer disease and related dementias. Alzheimer Disease and Associated Disorders, 11, 125-131.

Welsh-Bohmer, K. A., \& Warren, L. H. (2006). Neurodegenerative dementias. Dans K. A. Welsh-Bohmer \& D. K. Attix (Éds), Geriatric neuropsychology (pp. 56-88). New York: The Guildford Press.

Werthon, J. P., \& Monk, A. F. (2010). Problems people with dementia have with kitchen task: The challenge for pervasive computing. Interacting with Computers (22), 253266. 
Wickens, C. D. (1992). Engineering psychology and human performance ( $2^{\mathrm{e}}$ éd.) New York: Harper Collins.

Willis, S. L., Allen-Burge, R., Dolan, M. M., Bertrand, R. M., Yesavage, J., \& Taylor, J. L. (1998). Everyday problem solving among individuals with Alzheimer's disease. The Gerontologist, 38(5), 569-577.

Wilson, R. S., McCann, J. J., Li, Y., Aggarwal, N. T., Gilley, D. W., \& Evans, D. A. (2007). Nursing home placement, day care use, and cognitive decline in Alzheimer's disease. American Journal of Psychiatry, 164(666), 910-915.

Zec, R. F., \& Burkett, N. R. (2005). Neuropsychology of Alzheimer's disease and other dementias. Dans S. Bush \& T. Martin (Eds), Geriatric neuropsychology: Practice essentials (pp. 167-218). New York: Psycholgy Press. 
Appendice A

Formulaire de consentement 


\section{Formulaire d'informations et de consentement}

TITRE DU PROJET

Étude exploratoire descriptive de l'efficacité de différentes méthodes de guidage informatisé lors de la réalisation d'activités de préparation d'un café et d'une rôtie et d'emballage d'un cadeau auprès de trois participants atteints d'Alzheimer en stade modéré.

\section{CHERCHEURS RESPONSABLES DU PROJET DE RECHERCHE}

Madame Audrey Potvin, Ba. Psy., candidate au doctorat en psychologie

Madame Julie Bouchard, $\mathrm{PhD}$. professeure au Département des sciences de l'éducation et de psychologie de l'Université du Québec à Chicoutimi, co-directrice de l'étudiante

Monsieur Bruno Bouchard, $\mathrm{PhD}$, professeur au Département d'informatique et de mathématiques à l'Université du Québec à Chicoutimi, co-directeur de l'étudiante

\section{PRÉAMBULE}

Nous sollicitons votre participation (ou celle de la personne que vous représentez) à un projet de recherche. Cependant, avant d'accepter de participer à ce projet et de signer ce formulaire d'informations et de consentement, veuillez prendre le temps de lire, de comprendre et de considérer attentivement les renseignements qui suivent.

Ce formulaire peut contenir des mots que vous ne comprenez pas. Nous vous invitons à poser toutes les questions que vous jugerez utiles aux chercheurs responsables ou aux autres membres du personnel affectés au projet de recherche et à leur demander de vous expliquer tout mot ou renseignement qui n'est pas clair.

\section{DESCRIPTION DU PROJET}

La maladie d'Alzheimer (MA) est la cause la plus fréquente de démence dans les pays occidentaux industrialisés. Au Québec, la prévalence est de $5,1 \%$ chez les personnes âgées de plus de 65 ans. À titre d'exemple, au Canada, les coûts engendrés par la maladie d'Alzheimer atteignent environ 5,5 milliards de dollars à chaque année. Les déclins cognitifs dans la MA, qui touchent un ensemble de fonctions, demandent aux aidants une présence toujours croissante, ce qui augmente nécessairement leur fardeau et est en lien avec l'institutionnalisation. De ce fait, le Québec doit privilégier le maintien à domicile des personnes âgées plutôt que l'institutionnalisation et le gouvernement abonde dans ce sens pour des raisons aussi bien sociales qu'économiques. Dans la province, cette volonté s'est concrétisée avec la nouvelle politique gouvernementale sur le maintien à domicile des aînés. Afin de palier à cette demande de soins, des recherches s'effectuent actuellement afin de développer des agents intelligents qui seraient en mesure de soutenir les patients atteints de MA dans leurs activités quotidiennes et ainsi leur redonner une autonomie partielle tout en diminuant le fardeau des aidants. 
La réalisation de ce projet vise à réunir une banque de données sur des patients MA en stade modéré lors de la réalisation d'activités de la vie quotidienne (AVQ). Ces données serviront notamment à établir quel type de participant répond à quel type de guidage (ex : visuel, verbal, les deux) selon leur profil cognitif. Un guidage est une assistance informatisée en mesure de soutenir la réalisation d'AVQ par l'émission de suggestions lorsque les participants commettent des erreurs dans leur tâche. Un autre objectif consiste à compiler les réactions des participants face aux différents types de guidages, de même que de recueillir et classer les différents types d'erreurs commises lors des tâches effectuées.

Vous avez été sélectionné puisque vous répondez aux critères d'inclusion de la recherche (personne atteinte de maladie d'Alzheimer en stade modéré, le stade étant déterminé par l'échelle de Reisberg, hommes ou femmes, ayant moins de 90 ans, résidant au Saguenay-Lac-St-Jean) et que vous ne rencontrez pas les caractéristiques suivantes : troubles psychiatriques majeurs; et/ou des déficiences sensorielles majeures non-traitées; antécédents de maladies et/ou de dommages au cerveau; séquelles neurologiques conséquentes à des abus de drogue ou d'alcool (les états liés à ces précédentes caractéristiques devant être inscrits au dossier médical).

\section{DÉROULEMENT DE L'ÉTUDE}

Pour cette étude, nous sollicitons la participation de participants recrutés au Centre Cléophas-Claveau. En premier lieu, vous effectuerez une évaluation neuropsychologique afin d'évaluer les fonctions instrumentales et exécutives (si vous n'avez pas été évalué dans les derniers 6 mois). Dans le cas où vous auriez été évalué dans la période prescrite, une autorisation de communiquer les résultats vous sera demandée. Ensuite, vous serez appelé à vous présenter à l'UQAC (ou exceptionnellement à l'hôpital) à une reprise afin d'effectuer une tâche liée à la préparation de repas et une tâche non liée à la préparation des repas.

\section{AVANTAGES}

Vous (ou la personne que vous représentez) ne retirerez aucun bénéfice personnel de la participation à ce projet de recherche. Toutefois, les résultats obtenus pourraient contribuer à l'avancement des connaissances dans ce domaine. En effet, ces résultats devraient permettre aux cliniciens et aux chercheurs en domotique de mieux connaitre les profils neuropsychologiques des individus atteints d'Alzheimer et de les mettre en lien avec la technologie. Il sera alors possible de cibler les meilleures façons d'aider le patient dans la réalisation d'activités de la vie quotidienne. Les technologies pourront donc être plus adaptées aux demandes de services et ce, selon les besoins de la personne. 


\section{RISQUES ASSOCIÉS AU PROJET DE RECHERCHE}

Risques liés à l'évaluation neuropsychologique

Outre le temps requis pour l'évaluation, une certaine frustration ou la prise de conscience des déficits peuvent découler de l'évaluation. Si tel était le cas, le neuropsychologue en charge de l'évaluation interviendra selon la nécessité de la situation et une référence sera faite au psychologue du centre pour le suivi psychologique de la personne (qui est peut-être déjà entrepris).

\section{Risques liés à l'expérimentation}

Cette phase de l'étude comprend la réalisation d'activités de la vie quotidienne qui requiert l'utilisation de matériel de cuisine. Les participants seront en contact avec des ustensiles tels que couteau, cuillère, fourchette (en plastique afin d'éviter les risques de blessures). Une personne sera également sur place lors des expérimentations afin de veiller à la sécurité du participant en vérifiant l'utilisation correcte des instruments. De plus, la manipulation d'eau tiède sera étroitement surveillée. Une trousse de premiers soins sera présente en tout temps sur les lieux de l'expérimentation. Finalement, le temps pris pour l'expérimentation est un désavantage pour les participants en ce sens qu'il requiert des déplacements. Il est également entendu que la participation au projet n'aura aucun effet sur tout traitement médical dans le cadre du suivi habituel.

\section{CONFIDENTIALITÉ}

Durant votre participation (ou la participation de la personne que vous représentez) à ce projet, le chercheur responsable ainsi que son personnel recueilleront et consigneront, dans un dossier de recherche, les renseignements concernant les participants. Seuls les renseignements nécessaires pour répondre aux objectifs scientifiques de ce projet seront recueillis.

Ces renseignements peuvent comprendre les informations contenues dans vos dossiers médicaux concernant les résultats de l'évaluation neuropsychologique (s'il y a lieu), vos comportements lors de l'expérimentation, ainsi qu'un film de vos réalisations durant les tâches. Ce film sera réalisé de façon à ne pas filmer votre visage mais simplement les gestes que vous posez pour réaliser les tâches. Ce film sera numérisé et conservé de façon confidentielle sur le disque dur de l'ordinateur de l'un des directeurs de recherche à l'UQAC, tout en étant barré par mot de passe. Nous vous demandons également de cocher une case à la fin du formulaire permettant l'utilisation future de ces vidéos pour d'autres recherches. Ces dernières viseront à établir les profils d'erreurs et permettront de faire des simulations des séquences d'action par ordinateur. En aucun cas ces données ne permettront de vous identifier. Votre dossier peut aussi comprendre d'autres renseignements tels que votre nom, votre sexe, votre date de naissance, votre origine ethnique et votre scolarité (pour fin d'établissement de profil socio-démographique de la cohorte de participants).

Tous les renseignements recueillis demeureront strictement confidentiels dans les limites prévues par la loi. Afin de préserver votre identité et la confidentialité des 
renseignements, vous serez identifié par un numéro de code. La clé du code reliant votre nom à votre dossier de recherche sera conservée par l'un des professeurs responsables de la recherche dans un classeur barré de l'université. Nous conserverons les informations simplement afin de pouvoir les éliminer si le participant décide de mettre fin à l'expérimentation. Ainsi, si vous décidez de vous retirer avant les analyses, les données vous concernant seront détruites. Il est important de savoir que si les données sont déjà analysées, il sera impossible de les retirer de la base de données puisqu'elles seront alors confondues à toutes les autres.

Le chercheur responsable du projet utilisera les données à des fins de recherche dans le but de répondre aux objectifs scientifiques du projet décrits dans le formulaire d'information et de consentement. Suivant les normes de l'American Psychological Association (APA, 2001), toutes les données seront conservées pour une période de cinq ans suivant la dernière publication.

Les données pourront être publiées dans des revues spécialisées, présentées dans des congrès ou faire l'objet de discussions scientifiques, mais il ne sera pas possible de vous identifier.

À des fins de surveillance et de contrôle, votre dossier de recherche, s'il y a lieu, pourra être consulté par une personne mandatée par le Comité central d'éthique de la recherche du ministre de la Santé et des Services sociaux ou du Comité d'éthique de la recherche de l'UQAC. Ces organismes adhèrent à une politique de confidentialité.

$\grave{A}$ des fins de protection, notamment afin de pouvoir communiquer avec vous rapidement, votre nom et prénom, vos coordonnées et la date de début et de fin de votre participation au projet seront conservés pendant un an après la fin du projet dans un répertoire à part maintenu par les chercheurs responsables.

Vous aurez le droit de consulter votre dossier de recherche pour vérifier les renseignements recueillis et les faire rectifier au besoin, et ce, aussi longtemps que le chercheur responsable du projet ou l'établissement détiendront ces informations. Cependant, afin de préserver l'intégrité scientifique du projet, vous pourriez avoir accès à certaines de ces informations qu'une fois votre participation terminée.

\section{PARTICIPATION VOLONTAIRE ET DROIT DE RETRAIT}

Votre participation (ou la participation de la personne que vous représentez) à ce projet de recherche est volontaire. Vous êtes donc libre de refuser d'y participer. Vous pouvez également vous retirer de ce projet à n'importe quel moment, sans avoir à donner de raison, en faisant connaitre votre décision au chercheur responsable du projet ou à l'un des membres du personnel affectés au projet. Tant que vos données n'auront pas été analysées, elles pourront être détruites. 
Le chercheur responsable du projet de recherche, le Comité central d'éthique de la recherche du ministre de la Santé et des Services sociaux, le comité d'éthique de la recherche de l'UQAC ou l'organisme subventionnaire peuvent mettre fin à votre participation, sans votre consentement, si de nouvelles découvertes ou informations indiquent que votre participation au projet n'est plus dans votre intérêt, si vous ne respectez pas les consignes du projet de recherche ou s'il existe des raisons administratives d'abandonner le projet.

Toute nouvelle connaissance acquise durant le déroulement du projet qui pourrait affecter votre décision de continuer d'y participer vous sera communiquée sans délai verbalement et par écrit.

\section{COMPENSATION}

Vous ne recevrez pas de compensation financière pour votre participation à ce projet de recherche, les frais de déplacement seront cependant couverts.

\section{PERSONNES-RESSOURCES}

Si vous avez des questions concernant le projet de recherche ou pour signaler tout inconfort ressenti à la suite de votre participation, il est possible de contacter les responsables de la recherche, mais plus particulièrement Mme Audrey Potvin et Mme Julie Bouchard qui pourront vous guider vers des ressources appropriées. Vous pouvez les joindre aux endroits suivants :

- Audrey Potvin, étudiante responsable du projet, (418) 545-5011 poste 2308

- Julie Bouchard, professeure au Département des sciences de l'éducation et de psychologie de l'Université du Québec à Chicoutimi, au 555 boulevard de l'Université, Chicoutimi (Québec), G7H 2B1 ou au numéro de téléphone suivant (418) 545-5011 poste 5667

Pour toute question concernant l'éthique de cette recherche, vous pouvez contacter M. Jean-Pierre Béland, président du comité d'éthique de la recherche de l'UQAC au numéro suivant : 418-545-5011 \# 5219

\section{SURVEILLANCE DES ASPECTS ÉTHIOUES DU PROJET DE RECHERCHE}

Le Comité d'éthique de la recherche de l'UQAC a approuvé ce projet de recherche et en assure le suivi. De plus, il approuvera au préalable toute révision et toute modification apportée au formulaire d'information et de consentement et au protocole de recherche.

Pour toute information, vous pouvez communiquer avec le président du Comité central d'éthique de la recherche de l'UQAC soit avec M. Jean-Pierre Béland au 418-545-5011 \#5219. 


\section{CONSENTEMENT}

En ma qualité de participant ou de représentant légal, j'ai pris connaissance du formulaire d'information et de consentement. Je reconnais qu'on m'a expliqué le projet, qu'on a répondu à mes questions et qu'on m'a laissé le temps voulu pour prendre une décision.

Après réflexion, j'accepte de participer à ce projet de recherche aux conditions qui y sont énoncées. Une copie signée et datée du présent formulaire d'information et de consentement m'a été remise.

Nom du représentant légal

Signature

Date

Assentiment de la personne capable de comprendre la nature du projet, mais ayant un représentant légal

Assentiment verbal de la personne incapable de signer mais capable de comprendre la nature de ce projet: oui non

J'accepte que les données (où il est impossible de m'identifier) puissent être utilisées ultérieurement pour d'autres recherches sur la même problématique : oui non

Je comprends qu'on me demande l'accès à mon dossier médical pour obtenir seulement les résultats des évaluations neuropsychologiques et le diagnostic

Nom du témoin

$$
\text { Signature }
$$

Date

Je certifie qu'on a expliqué au participant et à son représentant légal les termes du présent formulaire d'information et de consentement, que l'on a répondu aux questions que le participant ou le représentant légal avait à cet égard et qu'on lui a clairement indiqué qu'il demeure libre de mettre un terme à sa participation au projet de recherche, et ce, sans préjudice. Je certifie que j'ai expliqué le projet de recherche au participant dans un langage adapté à son discernement, qu'il a compris et qu'il ne s'est pas opposé. Je m'engage à respecter tout refus.

Je m'engage, avec l'équipe de recherche, à respecter ce qui a été convenu au formulaire d'information et de consentement et à en remettre une copie signée et datée au participant ou au représentant légal.

Nom du chercheur

Fonction

Signature

Date 
Appendice B

Questionnaire sociodémographique 
Questionnaire sociodémographique

Date :

Code du participant :

Nom :

Prénom :

Sexe : F

M

Numéro de téléphone :

Date de naissance : /Jour /Mois /Année Âge :

Langue parlée à la maison :

Scolarité : Dernier diplôme complété :

Occupation antérieure :

Statut civil : Depuis :

Revenus brut :1) moins de $20000 \$$ 2) entre $20000 \$$ et $39999 \$ \square$

3) entre $40000 \$$ et $59999 \$ \square$

4) entre 60000 et $79999 \$ \square$

5) $80000 \$$ et plus $\square$

Demeurez-vous seul? Oui Non

Si non, avec qui demeurez-vous?

Recevez-vous de l'aide afin d'effectuer vos activités quotidiennes? Oui Non

Si oui, cette aide vient de : famille $\square \quad$ conjoint $\square \quad$ infirmière $\square$ autres (précisez)

Prenez vous des médicaments? Oui Non

Si oui, lesquels :

Êtes-vous fidèle à votre médication? Oui Non 


\section{Appendice $C$}

Description des interventions auprès du participant 
Description des interventions auprès du participant

\section{Interventions effectuées par l'assistant de recherche}

\section{Instructions du NAT}

- Rôtie et café

«Je vous demanderais de faire deux choses (montrez deux doigts) : faites une seule rôtie (pointez vers l'image de la tâche complétée) et faites une tasse de café instantané (pointez vers l'image de la tâche complétée). Tout ce dont vous aurez besoin pour ces deux tâches est là (faites un geste pour indiquer la table dans sa totalité). Vous devez faire une seule rôtie avec du beurre et de la confiture et une tasse de café instantané avec lait et sucre. Pouvez-vous, s'il vous plait, répéter ce que vous allez faire. " (Le participant répète les instructions ou indique d'une autre façon sa compréhension des directives.)

«Bien, s'il vous plait commencez. »

- Présent

"Je voudrais que vous emballiez un cadeau (pointez vers l'image de la tâche complétée). Utilisez ce dont vous aurez besoin dans ce qui est là (faites un geste pour indiquer la table dans sa totalité). Pouvez-vous, s'il vous plait, répéter ce que vous allez faire. » (Le participant répète les instructions ou indique d'une autre façon sa compréhension des directives.)

«Bien, s'il vous plait commencez. »

\section{Réponses aux questions des participants}

«Tout ce dont vous avez besoin est là. Faites de votre mieux.» ou

"Je ne peux vous dire comment faire. Faites de votre mieux. »

Le participant ne répond pas (ou ignore) deux guidages fournis à trente secondes d'intervalle

- L'assistant de recherche vient en aide au participant afin de réaliser l'étape en cours. Il lui dit: "Je vais vous aider. » Lorsque l'étape est terminée, l'assistant de recherche dit : "Maintenant, poursuivez (nom de la tâche) par vous-même. »

Interventions informatisées

\section{Après un délai de trente secondes de comportement non productif ou progressif}

Guidages informatisés 
Appendice D

Exemple d'ordre de présentation des méthodes de guidage 
Exemple d'ordre de présentation des méthodes de guidage

\begin{tabular}{|c|c|c|c|c|}
\hline \multirow{2}{*}{ Participant } & Visuel & Verbal & \multicolumn{2}{|c|}{ Audiovisuel } \\
\hline & CF $\quad$ EC & $\begin{array}{ll}\mathrm{CF} & \mathrm{EC}\end{array}$ & $\mathrm{CF}$ & $\mathrm{EC}$ \\
\hline $\mathrm{P} 1$ & 1 & 2 & & \\
\hline P2 & 3 & 1 & & \\
\hline P3 & 2 & 3 & & \\
\hline P4 & 1 & 2 & & \\
\hline P5 & 3 & 1 & & \\
\hline P6 & 2 & 3 & & 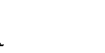 \\
\hline
\end{tabular}

CF : Tâche café et rôtie

EC : Tâche emballage d'un cadeau 


\section{Appendice $E$}

Étapes d'introduction des guidages dans la séquence : tâche café et rôtie 
Étapes d'introduction des guidages dans la séquence : tâche café et rôtie

- Chaque $\mathrm{X}$ correspond à un guidage introduit

- Les étapes précédées d'un astérisque représentent des étapes facultatives dans la séquence

$A=$ Guidage visuel

$\mathrm{B}=$ Guidage verbal

$\mathrm{C}=$ Guidage audiovisuel

\begin{tabular}{|l|l|l|l|l|l|l|l|l|l|}
\hline $\begin{array}{l}\text { Séquence d'action } \\
\text { café }\end{array}$ & \multicolumn{3}{|c|}{ P1 } & \multicolumn{3}{c|}{ P2 } & \multicolumn{3}{c|}{ P3 } \\
\hline & A & B & C & A & B & C & A & B & C \\
\hline 1. Prendre tasse & & & & & & & & & \\
\hline 2. Prendre café & & & $\mathrm{XX}$ & & & & & & \\
\hline 3. Ouvrir café & & & & & & & & & \\
\hline 4. Prendre cuillère & & & & & & & & & \\
\hline $\begin{array}{l}\text { 5. Mettre café } \\
\text { dans tasse }\end{array}$ & & & & & & & & & \\
\hline *Fermer café & & & & & & & & & \\
\hline 6. Saisir bouilloire & & $\mathrm{X}$ & & & & & & & \\
\hline 7. Verser eau & & & & & & & & & \\
\hline 8. Prendre sucrier & & & & & & $\mathrm{X}$ & & $\mathrm{X}$ & \\
\hline 9. Ouvrir sucrier & & & & & & & & & \\
\hline $\begin{array}{l}\text { 10. Mettre sucre } \\
\text { dans café avec } \\
\text { cuillère }\end{array}$ & & & & & & & & & \\
\hline *Fermer sucrier & & & & & & & & & \\
\hline 11. Prendre lait & & & & & & & & $\mathrm{X}$ & \\
\hline 12. Verser lait & & & & & & & & & \\
\hline *Mélanger & & & & & & & & & \\
\hline $\begin{array}{l}\text { Séquence d'action } \\
\text { rôtie }\end{array}$ & & & & & & & \\
\hline $\begin{array}{l}\text { 1. Prendre sac } \\
\text { pain }\end{array}$ & & & & & $\mathrm{X}$ & & & & \\
\hline 2. Ouvrir sac pain & & & & & & & & & \\
\hline $\begin{array}{l}\text { 3. Sortir tranche } \\
\text { pain }\end{array}$ & & & & & & & & & \\
\hline *Fermer sac pain & & & & & & & & & \\
\hline $\begin{array}{l}\text { 4. Mettre pain } \\
\text { dans grille-pain }\end{array}$ & $\mathrm{XX}$ & $\mathrm{XX}$ & & & & & $\mathrm{XX}$ & & \\
\hline
\end{tabular}




\begin{tabular}{|l|l|l|l|l|l|l|l|l|l|}
\hline \multirow{2}{*}{$\begin{array}{l}\text { Séquence d'action } \\
\text { rôtie (suite) }\end{array}$} & \multicolumn{3}{|c|}{ P1 } & \multicolumn{3}{c|}{ P2 } & \multicolumn{3}{|c|}{ P3 } \\
\hline & B & B & C & A & B & C & A & B & C \\
\hline $\begin{array}{l}\text { Mettre grille- } \\
\text { pain en fonction }\end{array}$ & & & & & & & & & \\
\hline $\begin{array}{l}\text { 6. Sortir pain du } \\
\text { grille-pain }\end{array}$ & & & & & & & & & \\
\hline 7. Prendre beurrier & & & & & & & & & \\
\hline 8. Ouvrir beurrier & & & & & & & & & \\
\hline $\begin{array}{l}\text { 9. Mettre beurre } \\
\text { sur couteau }\end{array}$ & & & & & & & & & \\
\hline 10. Tartiner beurre & & & & & & & & & \\
\hline *Fermer beurrier & & & & & & & & & \\
\hline $\begin{array}{l}\text { 11. Prendre } \\
\text { confiture }\end{array}$ & & & $\mathrm{X}$ & $\mathrm{XX}$ & $\mathrm{X}$ & $\mathrm{XX}$ & $\mathrm{XX}$ & & $\mathrm{X}$ \\
\hline 12. Ouvrir confiture & & & & & & & & & \\
\hline $\begin{array}{l}\text { 13. Prendre } \\
\text { confiture avec } \\
\text { cuillère/couteau }\end{array}$ & & & & & & & & & \\
\hline $\begin{array}{l}\text { 14. Tartiner } \\
\text { confiture }\end{array}$ & & & & & & & & & \\
\hline *Fermer confiture & & & & & & & & & \\
\hline \multicolumn{1}{c}{ Total } & 2 & 3 & 3 & 2 & 2 & 3 & 6 & 2 & 1 \\
\hline
\end{tabular}




\section{Appendice $F$}

Étapes d'introduction des guidages dans la séquence : tâche cadeau 
Étapes d'introduction des guidages dans la séquence : tâche cadeau

- Chaque $\mathrm{X}$ correspond à un guidage introduit

- Les étapes précédées d'un astérisque représentent des étapes facultatives dans la séquence

$\mathrm{A}=$ Guidage visuel

$\mathrm{B}=$ Guidage verbal

$\mathrm{C}=$ Guidage audiovisuel

\begin{tabular}{|l|l|l|l|l|l|l|l|l|l|}
\hline \multirow{2}{*}{$\begin{array}{c}\text { Séquence d'action } \\
\text { cadeau }\end{array}$} & \multicolumn{2}{|c|}{ P1 } & \multicolumn{3}{c|}{ P2 } & \multicolumn{3}{|c|}{ P3 } \\
\hline & A & B & C & A & B & C & A & B & C \\
\hline 1. Prendre boîte & & & & & & & & & \\
\hline 2. Prendre jouet & & & & $\mathrm{X} X$ & $\mathrm{XX}$ & & & & \\
\hline $\begin{array}{l}\text { 3. Envelopper } \\
\text { jouet }\end{array}$ & & & & & & & & & \\
\hline $\begin{array}{l}\text { 4. Mettre jouet } \\
\text { dans boîte }\end{array}$ & & & & & & & & & \\
\hline 5. Fermer boîte & & & & & & & & & \\
\hline $\begin{array}{l}\text { *Coller couvercle } \\
\text { bô̂te }\end{array}$ & & & & & & & & & \\
\hline $\begin{array}{l}\text { 6. Dérouler papier } \\
\text { emballage }\end{array}$ & & $\mathrm{XX}$ & $\mathrm{X}$ & & & & & & \\
\hline $\begin{array}{l}\text { 7. Mesurer papier } \\
\text { emballage }\end{array}$ & & & & & & & & & \\
\hline $\begin{array}{l}\text { 8. Couper papier } \\
\text { emballage }\end{array}$ & & & & & & & & & \\
\hline $\begin{array}{l}\text { 9. Plier rebord } \\
\text { dos) }\end{array}$ & & & & & & & & & \\
\hline 10. Coller dos & & & & & & & & & \\
\hline $\begin{array}{l}\text { 11. Plier première } \\
\text { extrémité }\end{array}$ & & & & & & & & & \\
\hline $\begin{array}{l}\text { 12. Coller première } \\
\text { extrémité }\end{array}$ & & & & & & & & & \\
\hline $\begin{array}{l}\text { *Recouper } \\
\text { première extrémité }\end{array}$ & & & & & & & & & \\
\hline $\begin{array}{l}\text { 13. Plier deuxième } \\
\text { extrémité }\end{array}$ & & & & & $\mathrm{XX}$ & $\mathrm{XX}$ & & & \\
\hline $\begin{array}{l}\text { 14. Coller } \\
\text { deuxième } \\
\text { extrémité }\end{array}$ & & & & & $\mathrm{XX}$ & & & & \\
\hline
\end{tabular}




\begin{tabular}{|c|c|c|c|c|c|c|c|c|c|}
\hline \multirow{2}{*}{$\begin{array}{l}\text { Séquence d'action } \\
\text { cadeau (suite) }\end{array}$} & \multicolumn{3}{|c|}{ P1 } & \multicolumn{3}{|c|}{$\mathbf{P 2}$} & \multicolumn{3}{|c|}{ P3 } \\
\hline & $\mathbf{A}$ & $\mathbf{B}$ & $\mathrm{C}$ & $\mathbf{A}$ & $\bar{B}$ & $\mathbf{C}$ & $\mathbf{A}$ & $\mathbf{B}$ & $\mathbf{C}$ \\
\hline $\begin{array}{l}\text { 15. Prendre sac de } \\
\text { choux }\end{array}$ & & $\mathrm{X}$ & $\mathrm{X}$ & $\mathrm{XX}$ & $\mathrm{XX}$ & $\mathrm{XX}$ & & & \\
\hline 16. Sortir un chou & & & & & & & & & \\
\hline $\begin{array}{l}\text { 17. Enlever papier } \\
\text { protecteur sur le } \\
\text { chou }\end{array}$ & X X & & & & & & & & \\
\hline 18. Coller le chou & & & & & & & & & \\
\hline Total & 2 & 3 & 2 & 4 & 8 & 4 & 0 & 0 & 0 \\
\hline
\end{tabular}




\section{Appendice $G$}

Modèle du traitement de l'information selon Wickens (1992) 
Modèle du traitement de l'information selon Wickens (1992)

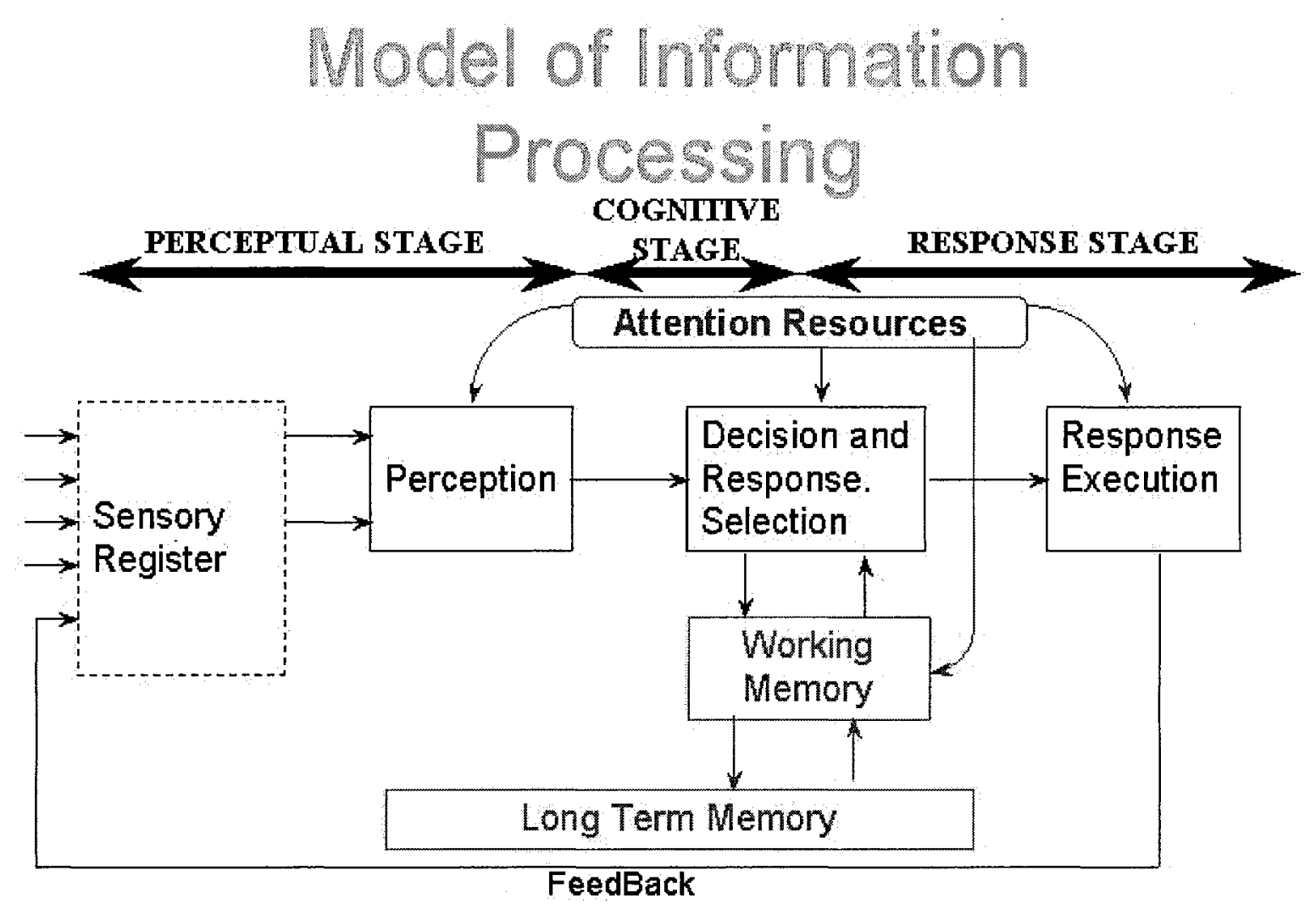

